\title{
Radiative and mechanical feedback into the molecular gas in the Large Magellanic Cloud
}

\section{30 Doradus $\star$}

\author{
M.-Y. Lee ${ }^{1,2,3}$, S. C. Madden ${ }^{2}$, F. Le Petit ${ }^{4}$, A. Gusdorf $f^{5,6}$, P. Lesaffre ${ }^{5,6}$, R. Wu$^{4}$, V. Lebouteiller ${ }^{2}$, \\ F. Galliano ${ }^{2}$, and M. Chevance ${ }^{7}$
}

\footnotetext{
${ }^{1}$ Max-Planck-Institut für Radioastronomie, Auf dem Hügel 69, 53121 Bonn, Germany e-mail: mlee@mpifr-bonn.mpg.de

2 AIM, CEA, CNRS, Université Paris-Saclay, Université Paris Diderot, Sorbonne Paris Cité, 91191 Gif-sur-Yvette, France

${ }^{3}$ Korea Astronomy and Space Science Institute, 776 Daedeokdae-ro, 34055 Daejeon, Republic of Korea

${ }^{4}$ LERMA, Observatoire de Paris, PSL Research University, CNRS, Sorbonne Université, 92190 Meudon, France

${ }^{5}$ Laboratoire de Physique de l'ENS, ENS, Université PSL, CNRS, Sorbonne Université, Université de Paris, Paris, France

${ }^{6}$ Observatoire de Paris, Université PSL, Sorbonne Université, LERMA, 75014 Paris, France

7 Astronomisches Rechen-Institut, Zentrum für Astronomie der Universität Heidelberg, Mönchhofstraße 12-14, 69120 Heidelberg, Germany
}

Received 5 February 2019 / Accepted 20 May 2019

\begin{abstract}
With an aim of probing the physical conditions and excitation mechanisms of warm molecular gas in individual star-forming regions, we performed Herschel SPIRE Fourier Transform Spectrometer (FTS) observations of 30 Doradus in the Large Magellanic Cloud. In our FTS observations, important far-infrared (FIR) cooling lines in the interstellar medium, including CO $J=4-3$ to $J=13-12$, [C I] $370 \mu \mathrm{m}$, and [N II] $205 \mu \mathrm{m}$, were clearly detected. In combination with ground-based CO $J=1-0$ and $J=3-2$ data, we then constructed CO spectral line energy distributions (SLEDs) on $\sim 10 \mathrm{pc}$ scales over a $\sim 60 \mathrm{pc} \times 60 \mathrm{pc}$ area and found that the shape of the observed CO SLEDs considerably changes across 30 Doradus. For example, the peak transition $J_{\mathrm{p}}$ varies from $J=6-5$ to $J=10-9$, while the slope characterized by the high-to-intermediate $J$ ratio $\alpha$ ranges from $\sim 0.4$ to $\sim 1.8$. To examine the source(s) of these variations in $\mathrm{CO}$ transitions, we analyzed the $\mathrm{CO}$ observations, along with [C II] $158 \mu \mathrm{m}$, [C I] $370 \mu \mathrm{m}$, [O I] $145 \mu \mathrm{m}, \mathrm{H}_{2} 0-0 \mathrm{~S}(3)$, and FIR luminosity data, using state-of-the-art models of photodissociation regions and shocks. Our detailed modeling showed that the observed $\mathrm{CO}$ emission likely originates from highly compressed (thermal pressure $P / k_{\mathrm{B}} \sim 10^{7}-10^{9} \mathrm{~K} \mathrm{~cm}^{-3}$ ) clumps on $\sim 0.7-2 \mathrm{pc}$ scales, which could be produced by either ultraviolet (UV) photons (UV radiation field $G_{\mathrm{UV}} \sim 10^{3}-10^{5}$ Mathis fields) or low-velocity C-type shocks (pre-shock medium density $n_{\text {pre }} \sim 10^{4}-10^{6} \mathrm{~cm}^{-3}$ and shock velocity $v_{\mathrm{s}} \sim 5-10 \mathrm{~km} \mathrm{~s}^{-1}$ ). Considering the stellar content in 30 Doradus, however, we tentatively excluded the stellar origin of $\mathrm{CO}$ excitation and concluded that low-velocity shocks driven by kiloparsec-scale processes (e.g., interaction between the Milky Way and the Magellanic Clouds) are likely the dominant source of heating for CO. The shocked CO-bright medium was then found to be warm (temperature $T \sim 100-500 \mathrm{~K}$ ) and surrounded by a UV-regulated low-pressure component $\left(P / k_{\mathrm{B}} \sim\right.$ a few $\left.\left(10^{4}-10^{5}\right) \mathrm{K} \mathrm{cm}^{-3}\right)$ that is bright in [C II] $158 \mu \mathrm{m},[\mathrm{C}$ I] $370 \mu \mathrm{m}$, [O I] $145 \mu \mathrm{m}$, and FIR dust continuum emission.
\end{abstract}

Key words. ISM: molecules - Magellanic Clouds - galaxies: ISM - infrared: ISM

\section{Introduction}

As a nascent fuel for star formation, molecular gas plays an important role in the evolution of galaxies (e.g., Kennicutt \& Evans 2012). The rotational transitions of carbon monoxide $(\mathrm{CO})^{1}$ have been the most widely used tracer of such a key component of the interstellar medium (ISM) and in particular enable the examination of the physical conditions of molecular gas in diverse environments (e.g., kinetic temperature $T_{\mathrm{k}} \sim 10-1000 \mathrm{~K}$ and density $n \sim 10^{3}-10^{8} \mathrm{~cm}^{-3}$ ) thanks to their large range of critical densities.

\footnotetext{
${ }^{\star}$ Herschel is an ESA space observatory with science instruments provided by European-led Principal Investigator consortia and with important participation from NASA.

1 In this paper, we focus on ${ }^{12} \mathrm{CO}$ and refer to it as $\mathrm{CO}$.
}

The diagnostic power of $\mathrm{CO}$ rotational transitions has been explored to a greater extent since the advent of the ESA Herschel Space Observatory (Pilbratt et al. 2010). The three detectors on board Herschel, PACS (Photodetector Array Camera and Spectrometer; Poglitsch et al. 2010), SPIRE (Spectral and Photometric Imaging Receiver; Griffin et al. 2010), and HIFI (Heterodyne Instrument for the Far Infrared; de Graauw et al. 2010), provide access to a wavelength window of $\sim 50-670 \mu \mathrm{m}$ and enable the study of CO spectral line energy distributions (SLEDs) up to the upper energy level $J_{\mathrm{u}}=50$ for Galactic and extragalactic sources including photodissociation regions (PDRs; e.g., Habart et al. 2010; Köhler et al. 2014; Stock et al. 2015; Joblin et al. 2018; $\mathrm{Wu}$ et al. 2018), protostars (e.g., Larson et al. 2015), infrared (IR) dark clouds (e.g., Pon et al. 2016), IR bright galaxies (e.g., Rangwala et al. 2011; Kamenetzky et al. 2012; Meijerink et al. 2013; Pellegrini et al. 2013; Papadopoulos et al. 2014; 

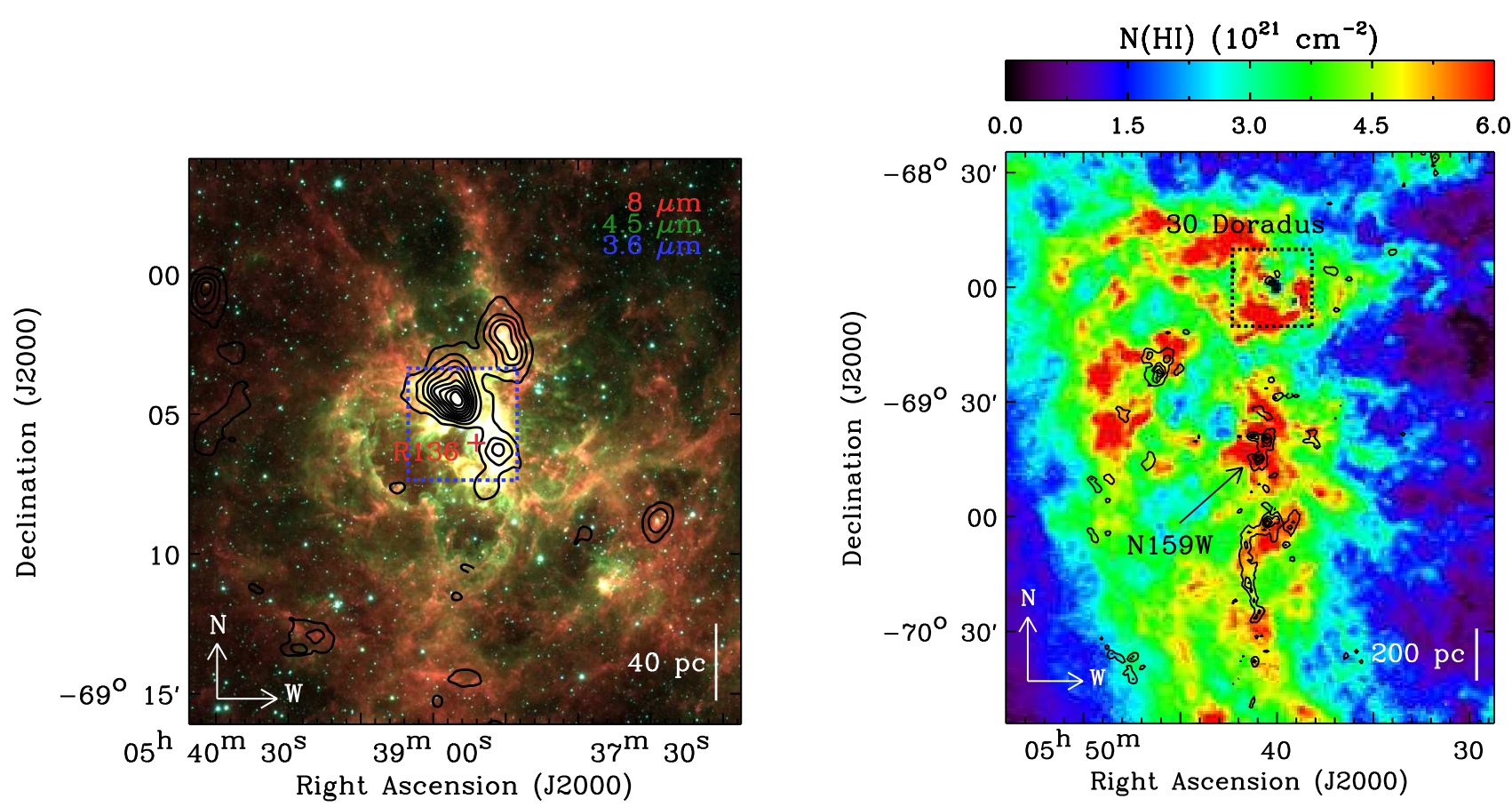

Fig. 1. Left: three-color composite image of 30 Doradus (Spitzer 3.6, 4.5, and $8 \mu \mathrm{m}$ in blue, green, and red; Meixner et al. 2006). The central star cluster R136 is marked with the red cross, and the FTS coverage is outlined in blue. Additionally, the $\mathrm{CO}(1-0)$ integrated intensity from the MAGMA survey (Wong et al. 2011; Sect. 3.4) is overlaid as the black contours with levels ranging from 10 to $90 \%$ of the peak (16.6 K km s$~^{-1}$ ) in steps of $10 \%$. Right: H I column density image from Kim et al. (1998). The MAGMA CO(1-0) integrated intensity is shown as the black contours ( 10 to $90 \%$ of the peak value, $39.5 \mathrm{~K} \mathrm{~km} \mathrm{~s}^{-1}$, in steps of $20 \%$ ), and the coverage of the left image is indicated by the black dashed box. This large H I structure, where 30 Doradus and N159W are located, corresponds to the southeastern H I overdensity region of the LMC (e.g., D'Onghia \& Fox 2016).

Rosenberg et al. 2014; Schirm et al. 2014; Mashian et al. 2015; $\mathrm{Wu}$ et al. 2015), and Seyfert galaxies (e.g., van der Werf et al. 2010; Hailey-Dunsheath et al. 2012; Israel et al. 2014). These studies have revealed the ubiquitous presence of warm molecular gas $\left(T_{\mathrm{k}} \gtrsim 100 \mathrm{~K}\right)$ and have proposed various radiative (e.g., UV photons, X-rays, and cosmic-rays) and mechanical (e.g., shocks) heating sources for $\mathrm{CO}$ excitation. As the dominant contributor to the total CO luminosity of galaxies ( $70 \%$; e.g., Kamenetzky et al. 2017), the warm CO is an important phase of the molecular medium. Understanding its physical conditions and excitation mechanisms is therefore critical to fully assess different molecular reservoirs and their roles in the evolution of galaxies.

While previous Herschel-based studies have considered various types of objects, they have primarily focused on either small-scale Galactic ( $\sim 1 \mathrm{pc}$ or smaller) or large-scale extragalactic $(\sim 1 \mathrm{kpc}$ or larger) sources. As recently pointed out by Indriolo et al. (2017), CO SLEDs are affected not only by heating sources, but also by a beam dilution effect, suggesting that it is important to examine a wide range of physical scales to comprehensively understand the nature of warm molecular gas in galaxies. To bridge the gaps left by previous studies and provide insight into the excitation mechanisms of warm $\mathrm{CO}$ on intermediate scales $(\sim 10-100 \mathrm{pc})$, we conducted Herschel SPIRE Fourier Transform Spectrometer (FTS) observations of several star-forming regions in the Large Magellanic Cloud (LMC; distance of $\sim 50 \mathrm{kpc}$ and metallicity of $\sim 1 / 2 Z_{\odot}$; e.g., Russell \& Dopita 1992; Schaefer 2008). The first of our LMC studies was Lee et al. (2016), where we analyzed Herschel observations of the N159W star-forming region along with complementary ground-based CO data at $\sim 10 \mathrm{pc}$ resolution. Specifically, we examined CO transitions from $J=1-0$ to $J=13-12(J=2-1$ not included) over a $\sim 40 \mathrm{pc} \times 40 \mathrm{pc}$ area using the nonlocal thermodynamic equilibrium (NLTE) radiative transfer model RADEX (van der Tak et al. 2007) and found that the CO-emitting gas in N159W is warm $\left(T_{\mathrm{k}} \sim 150-750 \mathrm{~K}\right)$ and moderately dense $(n \sim \mathrm{a}$ few $10^{3} \mathrm{~cm}^{-3}$ ). To investigate the origin of this warm molecular gas, we evaluated the impact of several radiative and mechanical heating sources and concluded that low-velocity C-type shocks $\left(\sim 10 \mathrm{~km} \mathrm{~s}^{-1}\right)$ provide sufficient energy for CO heating, while UV photons regulate fine-structure lines [C II] $158 \mu \mathrm{m},[\mathrm{C} \mathrm{I}] 370 \mu \mathrm{m}$, and [O I] $145 \mu \mathrm{m}$. High-energy photons and particles including X-rays and cosmic-rays were found not to be significant for CO heating.

In this paper, we extend our previous analyses to 30 Doradus (or 30 Dor), the most extreme starburst in the Local Universe. This starburst harbors more than 1000 OB-type and Wolf-Rayet (W-R) stars (Doran et al. 2013) and emits 500 times more ionizing photons than the Orion Nebula (Pellegrini et al. 2010), producing a giant $\mathrm{H}$ II region. In particular, 30 Doradus is primarily powered by the central super star cluster R136, which has an extremely high stellar surface density $\left(\gtrsim 10^{7} M_{\odot} \mathrm{pc}^{-3}\right.$; e.g., Selman \& Melnick 2013) along with the most massive stars known ( $\gtrsim 150 M_{\odot}$; e.g., Crowther et al. 2010). The star cluster R136 is surrounded by vast cavities and bubble-like structures, which were likely created by strong stellar winds and supernova explosions (SNe) with a total energy of $\sim 10^{52}$ erg (e.g., Chu \& Kennicutt 1994; Townsley et al. 2006a). All in all, these extraordinary star formation activities make 30 Doradus an ideal laboratory for examining the impact of radiative and mechanical feedback into the surrounding ISM. In Fig. 1, we show 30 Doradus and its surrounding environment (H I overdensity 
region where 30 Doradus and N159W are located) in several tracers of gas and dust.

This paper is organized as follows. In Sect. 2, we provide a summary of recent studies of 30 Doradus that are most relevant to our work. In Sects. 3 and 4, we present the multi-wavelength datasets used in our study and describe the spatial distribution of $\mathrm{CO}$ and $[\mathrm{C} \mathrm{I}]$ emission, as well as the observed CO SLEDs. In Sects. 5 and 6, we then employ state-of-the-art theoretical models of PDRs and shocks to examine the physical conditions and excitation mechanisms of $\mathrm{CO}$ in 30 Doradus. Finally, we summarize the results from our study in Sect. 7.

\section{Characteristics of 30 Doradus}

As noted in Sect. 1, 30 Doradus is one of the best-studied starforming regions. In this section, we summarize recent studies on 30 Doradus that are the most relevant to our work.

\subsection{Stellar content}

When it comes to stellar feedback, massive young stars are considered to be a major player; their abundant UV photons create $\mathrm{H}$ II regions and PDRs, while their powerful stellar winds sweep up the surrounding ISM into shells and bubbles. The latest view on the massive young stars in 30 Doradus was offered by the VLT-FLAMES Tarantula Survey (Evans et al. 2011), and we focus here on Doran et al. (2013) where the first systematic census of hot luminous stars was presented. In Doran et al. (2013), 1145 candidate hot luminous stars were identified based on $U B V$ band photometry, and $\sim 500$ of these stars were spectroscopically confirmed (including 469 OB-type stars and $25 \mathrm{~W}-\mathrm{R}$ stars). The total ionizing and stellar wind luminosities were then estimated to be $\sim 10^{52}$ photons s${ }^{-1}$ and $\sim 2 \times 10^{39} \mathrm{erg} \mathrm{s}^{-1}$, respectively, and $\sim 75 \%$ of these luminosities were found to come from the inner $20 \mathrm{pc}$ of 30 Doradus. This implies that stellar feedback is highly concentrated in the central cluster R136, where one third of the total W-R stars reside along with a majority of the most massive O-type stars. As for the age of stellar population, Doran et al. (2013) showed that the ionizing stars in 30 Doradus span multiple ages: mostly 2-5 Myr with an extension beyond $8 \mathrm{Myr}$.

\subsection{Properties of the neutral gas}

The impact of UV photons on the neutral gas in 30 Doradus was recently studied in detail by Chevance et al. (2016). The authors focused on Herschel PACS observations of traditional PDR tracers, including [C II] $158 \mu \mathrm{m}$ and [O I] $63 \mu \mathrm{m}$ and $145 \mu \mathrm{m}$, and found that [C II] and [O I] mostly arise from the neutral medium (PDRs), while [O I] $63 \mu \mathrm{m}$ is optically thick. The observed [C II] $158 \mu \mathrm{m}$ and [O I] $145 \mu \mathrm{m}$ were then combined with an image of IR luminosity to estimate the thermal pressure of $\sim(1-20) \times 10^{5} \mathrm{~K} \mathrm{~cm}^{-3}$ and the UV radiation of $\sim(1-300) \times 10^{2}$ Mathis fields (Mathis et al. 1983) via Meudon PDR modeling (Le Petit et al. 2006) on 12" scales ( 3 pc). In addition, the three-dimensional structure of PDRs was inferred based on a comparison between the stellar UV radiation field and the incident UV radiation field determined from PDR modeling: PDR clouds in 30 Doradus are located at a distance of $\sim 20-80 \mathrm{pc}$ from the central cluster R136.

As for the molecular ISM in 30 Doradus, Indebetouw et al. (2013) provided the sharpest view so far $\left(\sim 2^{\prime \prime}\right.$ or $\sim 0.5 \mathrm{pc}$ scales $)$ based on ALMA $\mathrm{CO}(2-1),{ }^{13} \mathrm{CO}(2-1)$, and $\mathrm{C}^{18} \mathrm{O}(2-1)$ observations of the 30Dor-10 cloud (Johansson et al. 1998). The main findings from their study are as follows: (1) CO emission mostly arises from dense clumps and filaments on $\sim 0.3-1 \mathrm{pc}$ scales; (2) interclump $\mathrm{CO}$ emission is minor, suggesting that there is considerable photodissociation of $\mathrm{CO}$ molecules by UV photons penetrating between the dense clumps; and (3) the mass of $\mathrm{CO}$ clumps does not change significantly with distance from $\mathrm{R} 136$. More excited CO lines in 30 Doradus (up to $J=6-5$ ) were recently analyzed by Okada et al. (2019), and we discuss this work in detail in Appendix E.

\subsection{High-energy photons}

The starburst 30 Doradus is a notable source of high-energy photons. For example, Townsley et al. (2006a,b) presented Chandra $\mathrm{X}$-ray observations of 30 Doradus, where a convoluted network of diffuse structures (associated with superbubbles and supernova remnants SNRs), as well as $\sim 100$ point sources (associated with O-type stars and W-R stars), were revealed. Thanks to the high spatial and spectral resolutions of the Chandra observations, the authors were able to investigate the properties of the X-ray-emitting hot plasma in detail, estimating a temperature of $(3-9) \times 10^{6} \mathrm{~K}$ and a surface brightness of $(3-130) \times$ $10^{31} \mathrm{erg} \mathrm{s}^{-1} \mathrm{pc}^{-2}$. In addition, Fermi $\gamma$-ray observations recently showed that 30 Doradus is the brightest source in the LMC with an emissivity of $\sim 3 \times 10^{-26}$ photons $\mathrm{s}^{-1} \mathrm{sr}^{-1}$ per hydrogen atom (Abdo et al. 2010). All in all, the presence of high-energy photons in 30 Doradus suggests that strong stellar winds and $\mathrm{SNe}$ have injected a large amount of mechanical energy into the surrounding ISM, driving shocks and accelerating particles.

\section{Data}

In this section, we present the data used in our study. Some of the main characteristics of the datasets, including rest wavelengths, angular resolutions, and sensitivities, are listed in Table 1.

\subsection{Herschel SPIRE spectroscopic data}

The starburst 30 Doradus was observed with the SPIRE FTS in the high spectral resolution, intermediate spatial sampling mode (Obs. IDs: 1342219550, 1342257932, and 1342262908). The FTS consists of two bolometer arrays, SPIRE Long Wavelength (SLW) and SPIRE Short Wavelength (SSW), which cover the wavelength ranges of 303-671 $\mu \mathrm{m}$ and 194-313 $\mu \mathrm{m}$, respectively. Depending on wavelength, the FTS beam size ranges from $17^{\prime \prime}$ to $42^{\prime \prime}$ (corresponding to $4-10 \mathrm{pc}$ at the distance of the LMC; Makiwa et al. 2013; Wu et al. 2015). The SLW and SSW comprise 19 and 37 hexagonally packed detectors, respectively, which cover approximately $3^{\prime} \times 3^{\prime}$. In the intermediate spatial sampling mode, these bolometer arrays are moved in a fourpoint jiggle with one beam spacing, resulting in sub-Nyquistsampled data. We note that spectral lines are not resolved in our observations due to the insufficient frequency resolution of $\Delta f=1.2 \mathrm{GHz}$ (corresponding to the velocity resolution of $\Delta v \sim$ $230-800 \mathrm{~km} \mathrm{~s}^{-1}$ across the SLW and SSW).

To derive integrated intensity images and their uncertainties, we essentially followed Lee et al. (2016) and $\mathrm{Wu}$ et al. (2015) and summarize our procedure here. First of all, we processed the FTS data using the Herschel Interactive Processing Environment (HIPE) version 11.0, along with the SPIRE calibration version 11.0 (Fulton et al. 2010; Swinyard et al. 2014). As an example, the processed spectra from two central SLW and SSW detectors are presented in Fig. 2, with the locations of the spectral lines observed with the SPIRE FTS. We then performed line 
Table 1. Spectral line and dust continuum data in our study.

\begin{tabular}{|c|c|c|c|c|c|c|}
\hline Transition & $\begin{array}{l}\text { Rest wavelength } \\
(\mu \mathrm{m})\end{array}$ & $\begin{array}{l}E_{\mathrm{u}}^{(a)} \\
(\mathrm{K})\end{array}$ & $\begin{array}{c}F W H M^{(b)} \\
\left({ }^{\prime \prime}\right)\end{array}$ & $\begin{array}{c}\sigma_{\mathrm{s}, \mathrm{med}}(c),(d),(e) \\
\left(10^{-11} \mathrm{~W} \mathrm{~m}^{-2} \mathrm{sr}^{-1}\right)\end{array}$ & $\begin{array}{c}\sigma_{\mathrm{f}, \mathrm{med}}(f),(d),(e) \\
\left(10^{-11} \mathrm{~W} \mathrm{~m}^{-2} \mathrm{sr}^{-1}\right)\end{array}$ & Reference \\
\hline${ }^{12} \mathrm{CO} J=1-0$ & 2600.8 & 6 & 45 & 0.1 & 0.1 & (1) \\
\hline${ }^{12} \mathrm{CO} \mathrm{J}=3-2$ & 867.0 & 33 & 22 & 2.2 & 5.1 & (2) \\
\hline${ }^{12} \mathrm{CO} \mathrm{J}=4-3$ & 650.3 & 55 & 42 & 24.0 & 26.5 & (3) \\
\hline${ }^{12} \mathrm{CO} \mathrm{J}=5-4$ & 520.2 & 83 & 34 & 15.9 & 20.7 & (3) \\
\hline${ }^{12} \mathrm{CO} \mathrm{J}=6-5$ & 433.6 & 116 & 29 & 9.2 & 16.7 & (3) \\
\hline${ }^{12} \mathrm{CO} \mathrm{J}=7-6$ & 371.7 & 155 & 33 & 7.4 & 18.4 & (3) \\
\hline${ }^{12} \mathrm{CO} \mathrm{J}=8-7$ & 325.2 & 199 & 33 & 18.7 & 25.3 & (3) \\
\hline${ }^{12} \mathrm{CO} \mathrm{J}=9-8$ & 289.1 & 249 & 19 & 25.2 & 30.2 & (3) \\
\hline${ }^{12} \mathrm{CO} J=10-9$ & 260.2 & 304 & 18 & 27.6 & 30.6 & (3) \\
\hline${ }^{12} \mathrm{CO} J=11-10$ & 236.6 & 365 & 17 & 28.7 & 32.2 & (3) \\
\hline${ }^{12} \mathrm{CO} J=12-11$ & 216.9 & 431 & 17 & 26.6 & 30.3 & (3) \\
\hline${ }^{12} \mathrm{CO} J=13-12$ & 200.3 & 503 & 17 & 39.8 & 43.0 & (3) \\
\hline$[\mathrm{C} \mathrm{I}]{ }^{3} P_{1}-{ }^{3} P_{0}$ & 609.1 & 24 & 38 & 28.3 & 29.2 & (3) \\
\hline$[\mathrm{C} \mathrm{I}]{ }^{3} P_{2}-{ }^{3} P_{1}$ & 370.4 & 62 & 33 & 7.0 & 7.9 & (3) \\
\hline$[\mathrm{C}$ II $]{ }^{2} P_{3 / 2}-{ }^{2} P_{1 / 2}$ & 157.7 & 91 & 12 & 294.6 & 8092.0 & (4) \\
\hline [O I $]{ }^{3} P_{0}-{ }^{3} P_{1}$ & 145.5 & 327 & 12 & 110.2 & 788.0 & (4) \\
\hline$[\mathrm{N}$ II $]{ }^{3} P_{1}-{ }^{3} P_{0}$ & 205.2 & 70 & 17 & 43.6 & 99.0 & (3) \\
\hline $\mathrm{H}_{2} 0-0 \mathrm{~S}(3)$ & 9.7 & 2504 & 6 & - & 1036.0 & $(3,5)$ \\
\hline FIR & $60-200$ & - & 42 & - & $2.9 \times 10^{6}$ & $(3,4)$ \\
\hline
\end{tabular}

Notes. ${ }^{(a)}$ Upper level energy. ${ }^{(b)}$ Angular resolution of the original data. ${ }^{(c)}$ Median $\sigma_{\mathrm{s}}$ (statistical $1 \sigma$ uncertainty) on $42^{\prime \prime}$ scales. ${ }^{(d)} \mathrm{CO}(1-0)$ is exceptionally on $45^{\prime \prime}$ scales. ${ }^{(e)}$ All pixels are considered. ${ }^{(f)}$ Median $\sigma_{\mathrm{f}}$ (final $1 \sigma$ uncertainty; statistical and calibration errors added in quadrature) on $42^{\prime \prime}$ scales.

References. (1) Wong et al. (2011); (2) Minamidani et al. (2008); (3) this work; (4) Chevance et al. (2016); (5) Indebetouw et al. (2009).

measurement of point-source calibrated spectra for each transition, where a linear combination of parabola and sinc functions was adopted to model the continuum and the emission line. The continuum-subtracted spectra were eventually projected onto a $5^{\prime} \times 5^{\prime}$ common grid with a pixel size of $15^{\prime \prime}$ to construct a spectral cube. Finally, the integrated intensity $\left(I_{\mathrm{CO}}, I_{\mathrm{CI}}\right.$, or $\left.I_{\mathrm{NII}}\right)$ was derived by carrying out line measurement of the constructed cube, and its final $1 \sigma$ uncertainty $\left(\sigma_{\mathrm{f}}\right)$ was estimated by summing two sources of error in quadrature, $\sigma_{\mathrm{f}}=\sqrt{\sigma_{\mathrm{s}}^{2}+\sigma_{\mathrm{c}}^{2}}$, where $\sigma_{\mathrm{s}}$ is the statistical error derived from line measurement and $\sigma_{\mathrm{c}}$ is the calibration error of $10 \%$ (SPIRE Observers' Manual ${ }^{2}$ ).

Throughout our study, the FTS data were frequently combined with other tracers of gas and dust. To compare the different datasets at a common angular resolution, we then smoothed the FTS images to $42^{\prime \prime}$, which corresponds to the FWHM of the FTS $\mathrm{CO}(4-3)$ observations, by employing the kernels from Wu et al. (2015). These kernels were created based on the fitting of a twodimensional Hermite-Gaussian function to the FTS beam profile, taking into account the complicated dependence on wavelength. In addition, the smoothed images were rebinned to have a final pixel size of $30^{\prime \prime}$, which roughly corresponds to the jiggle spacing of the SLW observations. We present the resulting integrated intensity maps in Fig. 3 and Appendix A and refer to Lee et al. (2016) and $\mathrm{Wu}$ et al. (2015) for full details on the data reduction and map-making procedures. Line detections in our FTS observations are discussed in Sect. 4.1.

We note that high-resolution spectra of $\mathrm{CO}(4-3), \mathrm{CO}(7-6)$, and [C I] $370 \mu \mathrm{m}\left(\sim 25^{\prime \prime}-40^{\prime \prime}\right)$ were previously obtained for 30 Doradus by Pineda et al. (2012) with the NANTEN2

\footnotetext{
2 http://herschel.esac.esa.int/Docs/SPIRE/html/spire_ om.html
}

telescope. The authors performed the observations as a single pointing toward $(\alpha, \delta)_{\mathrm{J} 2000}=\left(05^{\mathrm{h}} 38^{\mathrm{m}} 48.6^{\mathrm{s}},-69^{\circ} 04^{\prime} 43.2^{\prime \prime}\right)$, and we found that the NANTEN2-to-FTS ratios of the integrated intensities for this position are $\lesssim 1.2$, suggesting that our intensity measurements are consistent with Pineda et al. (2012) within $1 \sigma$ uncertainties.

\subsection{Herschel PACS spectrosopic data}

Following Lee et al. (2016), we used PACS [C II] $158 \mu \mathrm{m}$ and [O I] $145 \mu \mathrm{m}$ data of 30 Doradus. These data were first presented in Chevance et al. (2016), and we here provide a brief summary on the observations and data reduction. We note that [O I] $63 \mu \mathrm{m}$ was not used for our study, since the line is optically thick throughout the mapped region (e.g., [O I] $145 \mu \mathrm{m}$-to-[O I] $63 \mu \mathrm{m}$ ratio $>0.1$; Tielens \& Hollenbach 1985; Chevance et al. 2016).

The starburst 30 Doradus was mapped with the PACS spectrometer in the unchopped scan mode (Obs. IDs: 1342222085 to 1342222097 and 1342231279 to 1342231285 ). As an integral field spectrometer, the PACS consists of 25 (spatial) $\times 16$ (spectral) pixels and covers $51-220 \mu \mathrm{m}$ with a field of view of 47" $\times 47$ " (Poglitsch et al. 2010). The [C II] $158 \mu \mathrm{m}$ and [O I] $145 \mu \mathrm{m}$ fine-structure lines were observed in 31 and 11 raster positions, respectively, covering approximately $4^{\prime} \times 5^{\prime}$ over the sky. The beam size of the spectrometer at $160 \mu \mathrm{m}$ is $12^{\prime \prime}$ (PACS Observers' Manual ${ }^{3}$ ).

The obtained observations were reduced using the HIPE version 12.0 (Ott 2010) from Level 0 to Level 1. The reduced

\footnotetext{
3 http://herschel.esac. esa.int/Docs/PACS/html/pacs_om. html
} 

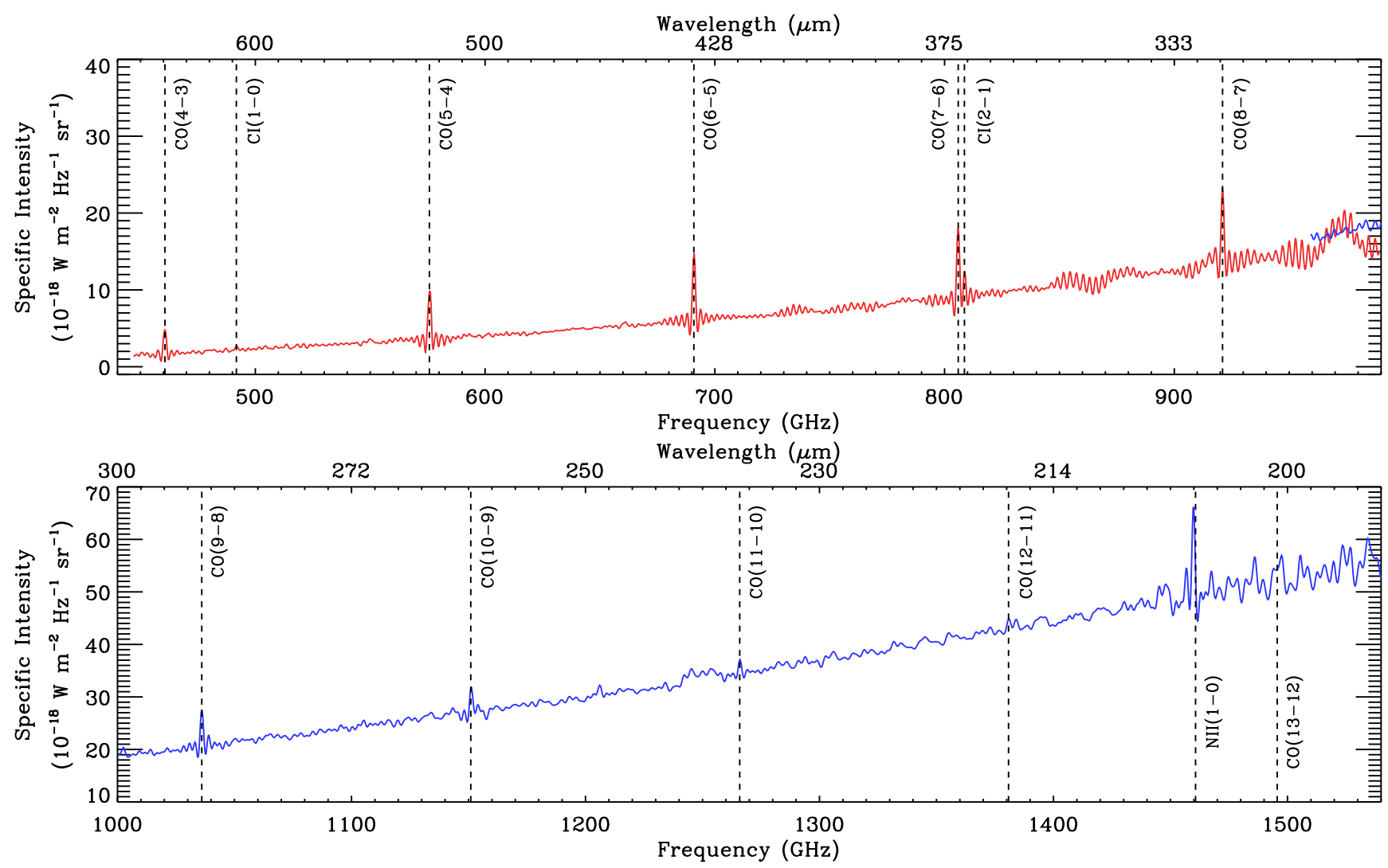

Fig. 2. Point-source calibrated spectra from two central detectors, SLWC3 (red) and SSWD4 (blue). These spectra are from the first jiggle position of the Obs. ID $=1342219550$, and the locations of the two detectors are shown as the yellow and orange crosses in Fig. 3. Additionally, the spectral lines observed with the SPIRE FTS are indicated by the black dashed lines. We note that no further data processing (e.g., baseline subtraction and smoothing) was done for the spectral lines here, which are at their original angular resolutions (e.g., Table 1).

cubes were then processed with PACSman (Lebouteiller et al. 2012) to derive integrated intensity maps. In essence, each spectrum was modeled with a combination of polynomial (baseline) and Gaussian (emission line) functions, and the measured line fluxes were projected onto a common grid with a pixel size of $3^{\prime \prime}$. The final $1 \sigma$ uncertainty in the integrated intensity was then estimated by adding the statistical error from line measurement/map projection and the calibration error of $22 \%$ in quadrature. For details on the observations, as well as the data-reduction and map-making procedures, we refer to Lebouteiller et al. (2012), Cormier et al. (2015), and Chevance et al. (2016).

In our study, the original PACS images were smoothed and rebinned to match the FTS resolution (42") and pixel size $\left(30^{\prime \prime}\right)$. This smoothing and rebinning procedure resulted in a total of 13 common pixels to work with (e.g., Fig. 7; mainly limited by the small coverage of the [O I] $145 \mu \mathrm{m}$ data), over which [C II] $158 \mu \mathrm{m}$ and [O I] $145 \mu \mathrm{m}$ were clearly detected with $S / N_{\mathrm{s}} \gg 5$.

\subsection{Spitzer IRS $\mathrm{H}_{2}$ data}

In addition, we made use of Spitzer InfraRed Spectrograph (IRS) observations of $\mathrm{H}_{2} \mathrm{O}-\mathrm{O} \mathrm{S}(3)$ in 30 Doradus. These observations were initially presented in Indebetouw et al. (2009), and we re-processed them as follows mainly to match the FTS resolution and pixel size. First, Basic Calibrated Data (BCD) products were downloaded from the Spitzer Heritage Archive (SHA), and exposures were cleaned with IRSclean ${ }^{4}$ and combined using

\footnotetext{
4 http://irsa.ipac.caltech.edu/data/SPITZER/docs/ dataanalysistools/tools/irsclean/
}

SMART $^{5}$ (Higdon et al. 2004; Lebouteiller et al. 2010). The data were then imported into CUBISM (Smith et al. 2007) for further cleaning and to build a calibrated data cube with pixel sizes of $2^{\prime \prime}$ and $5^{\prime \prime}$ for the Short-Low (SL) and Long-Low (LL) modules.

To produce a $\mathrm{H}_{2} \mathrm{O}-0 \mathrm{~S}(3)$ map, we performed a Monte Carlo simulation where 100 perturbed cubes were created based on the calibrated data cube. These cubes were then convolved and resampled to obtain a resolution of $42^{\prime \prime}$ and a pixel size of $30^{\prime \prime}$, and spectral line fitting was performed using LMFIT (Newville et al. 2014) for each cube. Finally, the line flux and associated uncertainty were calculated for each pixel using the median and median absolute deviation of the 100 measured flux values. While the resulting $\mathrm{H}_{2}$ map is as large as the FTS CO maps, we found that the observations were not sensitive: only five pixels have detections with $S / N_{\mathrm{s}} \sim 5$.

\subsection{Ground-based CO data}

We complemented our FTS CO observations with ground-based $\mathrm{CO}(1-0)$ and (3-2) data. The $\mathrm{CO}(1-0)$ data were taken from the MAGellanic Mopra Assessment (MAGMA) survey (Wong et al. 2011), where the $22 \mathrm{~m}$ Mopra telescope was used to map $\mathrm{CO}(1-0)$ in the LMC on $45^{\prime \prime}$ scales. Meanwhile, the $\mathrm{CO}(3-2)$ data were obtained by Minamidani et al. (2008) on 22" scales using the $10 \mathrm{~m}$ Atacama Submillimeter Telescope Experiment (ASTE) telescope. For both datasets, the final uncertainties in the integrated intensities were estimated in a similar manner to that used for our FTS CO observations: adding the statistical

\footnotetext{
5 http://irs.sirtf.com/IRS/SmartRelease
} 


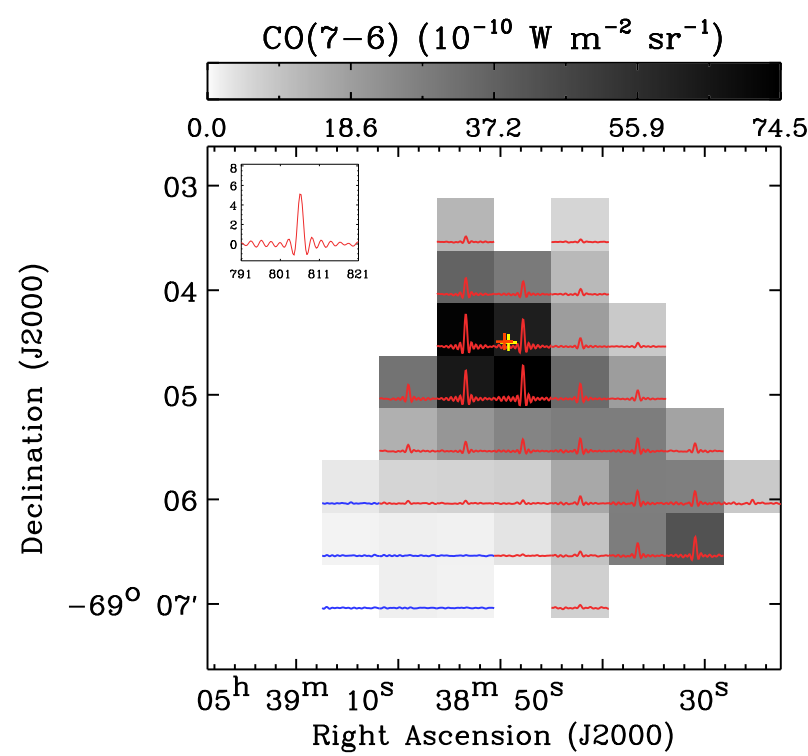

Fig. 3. $\mathrm{CO}(7-6)$ integrated intensity image $\left(F W H M=42^{\prime \prime}\right.$; pixel size $\left.=30^{\prime \prime}\right)$. In our FTS observations, $\mathrm{CO}(7-6)$ is one of the brightest and most sensitive transitions (Table 1; Sect. 4). Over the grayscale image, the spectrum of each pixel is overlaid, the $x$ - and $y$-axis ranges of which (in $\mathrm{GHz}$ and $10^{-18} \mathrm{~W} \mathrm{~m}^{-2} \mathrm{~Hz}^{-1} \mathrm{sr}^{-1}$ ) are indicated in the top left corner with an example spectrum. This spectrum is from the pixel that was observed with the two central detectors SLWC3 and SSWD4 (yellow and orange crosses) of the first jiggle observation of the Obs. $\mathrm{ID}=1342219550$. The spectra in red and blue represent detections and nondetections based on our threshold of statistical signal-to-noise ratio $\left(S / N_{\mathrm{s}}\right)=5$ (Sect. 4.1)

error derived from the root-mean-square (rms) noise per channel and the calibration error (25 and $20 \%$ for $\mathrm{CO}(1-0)$ and $\mathrm{CO}(3-2)$ respectively; Lee et al. 2016) in quadrature. We smoothed and rebinned the $\mathrm{CO}(1-0)^{6}$ and $\mathrm{CO}(3-2)$ maps to match the FTS data, leading to 31 and 26 pixels to work with, respectively. Among these pixels, the majority (22 and 25 pixels for $\mathrm{CO}(1-$ 0 ) and $\mathrm{CO}(3-2)$ respectively) had clear detections with $S / N_{\mathrm{s}}>5$ (e.g., Fig. 5).

\subsection{Derived dust and IR continuum properties}

Finally, we used the dust and IR continuum properties of 30 Doradus that were first estimated by Chevance et al. (2016) at $12^{\prime \prime}$ resolution based on the dust spectral energy distribution (SED) model of Galliano (2018). The Galliano (2018) SED model employs the hierarchical Bayesian approach and considers realistic optical properties, stochastic heating, and the mixing of physical conditions in observed regions. For our analyses, we essentially followed Chevance et al. (2016) and constrained the FIR luminosity $\left(60-200 \mu \mathrm{m} ; L_{\mathrm{FIR}}\right)$ and $V$-band dust extinction $\left(A_{\mathrm{V}}\right)$ over the FTS coverage on $42^{\prime \prime}$ scales. In our spatially resolved modeling of dust SEDs covering mid-IR to sub-millimeter, the amorphous carbon (AC) composition was considered along with the following free parameters: the total dust mass $\left(M_{\text {dust }}\right)$, PAH (polycyclic aromatic hydrocarbon)-todust mass ratio $\left(f_{\mathrm{PAH}}\right)$, index for the power-law distribution of starlight intensities $\left(\alpha_{U}\right)$, lower cut-off for the power-law distribution of starlight intensities $\left(U_{\min }\right)$, range of starlight intensities

6 In this paper, we used the $\mathrm{CO}(1-0)$ data at the original resolution of $45^{\prime \prime}$, which is quite close to the FTS resolution of $42^{\prime \prime}$, with a rebinned pixel size of $30^{\prime \prime}$.
$(\Delta U)$, and mass of old stars $\left(M_{\text {star }}\right)$. For details on our dust SED modeling, we refer to Galliano (2018).

\section{Results}

In this section, we mainly discuss the observed properties of the FTS lines, with a particular emphasis on $\mathrm{CO}$ and [C I] emission. The spectra and integrated intensity images of the FTS lines are presented in Fig. 3 and Appendix A.

\subsection{Spatial distribution of $\mathrm{CO}$ and $\left[\mathrm{C}_{\mathrm{I}} \mathrm{]}\right.$ emission}

Following Lee et al. (2016), we consider spectra with $S / N_{\mathrm{s}}$ (integrated intensity divided by $\left.\sigma_{\mathrm{s}}\right)>5$ as detections and group $\mathrm{CO}$ transitions into three categories: low- $J$ for $J_{\mathrm{u}} \leq 5$, intermediate- $J$ for $6 \leq J_{\mathrm{u}} \leq 9$, and high- $J$ for $J_{\mathrm{u}} \geq 10$. In our FTS observations, all CO transitions from $J=4-3$ to $J=13-12$, as well as [C I] $370 \mu \mathrm{m}$, were clearly detected. The sensitivity at $\sim 500 \mathrm{GHz}$ on the other hand was not good enough for [C I] $609 \mu \mathrm{m}$ to be detected.

In general, we found that $\mathrm{CO}(J=1-0$ to $13-12 ; J=2-1$ not included) and [C I] $370 \mu \mathrm{m}$ emission lines are distributed along the northern and southern lobes around R136, with primary and secondary peaks at $(\alpha, \delta)_{\mathrm{J} 2000} \sim\left(05^{\mathrm{h}} 38^{\mathrm{m}} 51^{\mathrm{s}},-69^{\circ} 04^{\prime} 38^{\prime \prime}\right)$ and $\left(05^{\mathrm{h}} 38^{\mathrm{m}} 38^{\mathrm{s}},-69^{\circ} 06^{\prime} 08^{\prime \prime}\right)$ (Fig. 4). This overall morphology is similar to that of PDR tracers, such as [C II] $158 \mu \mathrm{m},[\mathrm{O} \mathrm{I}]$ $145 \mu \mathrm{m}$, and PAH emission (Chevance et al. 2016). A close examination however revealed that detailed distributions are slightly different between the transitions. For example, the region between the northern and southern lobes, $(\alpha, \delta)_{\mathrm{J} 2000} \sim$ $\left(05^{\mathrm{h}} 38^{\mathrm{m}} 45^{\mathrm{s}},-69^{\circ} 05^{\prime} 30^{\prime \prime}\right)$, becomes bright in intermediateand high- $J \mathrm{CO}$ emission, resulting in the declining correlation between $\mathrm{CO}$ lines and fine-structure lines. Specifically, we found that the Spearman rank correlation coefficient remains high $(\rho \sim$ $0.8-0.9)$ for [C II] $158 \mu \mathrm{m}$ and CO from $J=1-0$ to $8-7(J=2-1$ not included), while being low for $J=9-8$ and 10-9 $(\rho=0.4$ and 0.1 ). Furthermore, [C I] $370 \mu \mathrm{m}$ was found to be strongly correlated with [C II] $158 \mu \mathrm{m}(\rho=0.9)$. For these estimates, we only considered detections and transitions with a sufficient number of detections. The decreasing correlation between [C II] $158 \mu \mathrm{m}$ and $\mathrm{CO}$ mainly results from the mid-region becoming bright in intermediate- and high- $J$ CO lines, indicating spatial variations in CO SLEDs (Sect. 4.2). To illustrate this result, we show CO $J=5-4$ and 10-9 along with [C II] $158 \mu \mathrm{m}$ in Fig. 4.

\subsection{Observed CO spectral line energy distributions}

The observed CO SLEDs of 30 Doradus are presented in Fig. 5. To construct these CO SLEDs, we first combined the FTS integrated intensity images with the ground-based $\mathrm{CO}(1-0)$ and (3-2) data at the common resolution of $42^{\prime \prime}$. We then used different colors to indicate the CO SLEDs with different peak transitions (black, green, and red for $J_{\mathrm{p}} \geq 9-8, J_{\mathrm{p}}<9-8$, and uncertain $J_{\mathrm{p}} ; J_{\mathrm{p}}=$ transition where a CO SLED peaks) and marked the location of each pixel relative to R136.

Figure 5 clearly shows that the shape of the CO SLEDs changes over the mapped region of $4^{\prime} \times 4^{\prime}(\sim 60 \mathrm{pc} \times 60 \mathrm{pc})$. For example, the majority (12 out of the total 21 pixels with certain $J_{\mathrm{p}}$ ) peak at $J=9-8$ or $10-9$, while some have $6-5 \leq J_{\mathrm{p}}<$ 9-8. In addition, the slope of the CO SLEDs varies substantially. To quantify the variation in the slopes, we then defined the high-to-intermediate- $J \mathrm{CO}$ ratio $(\alpha)$ as follows,

$\alpha=\frac{I_{\mathrm{CO}}(J=9-8)+I_{\mathrm{CO}}(J=10-9)+I_{\mathrm{CO}}(J=11-10)}{I_{\mathrm{CO}}(J=5-4)+I_{\mathrm{CO}}(J=6-5)+I_{\mathrm{CO}}(J=7-6)}$, 

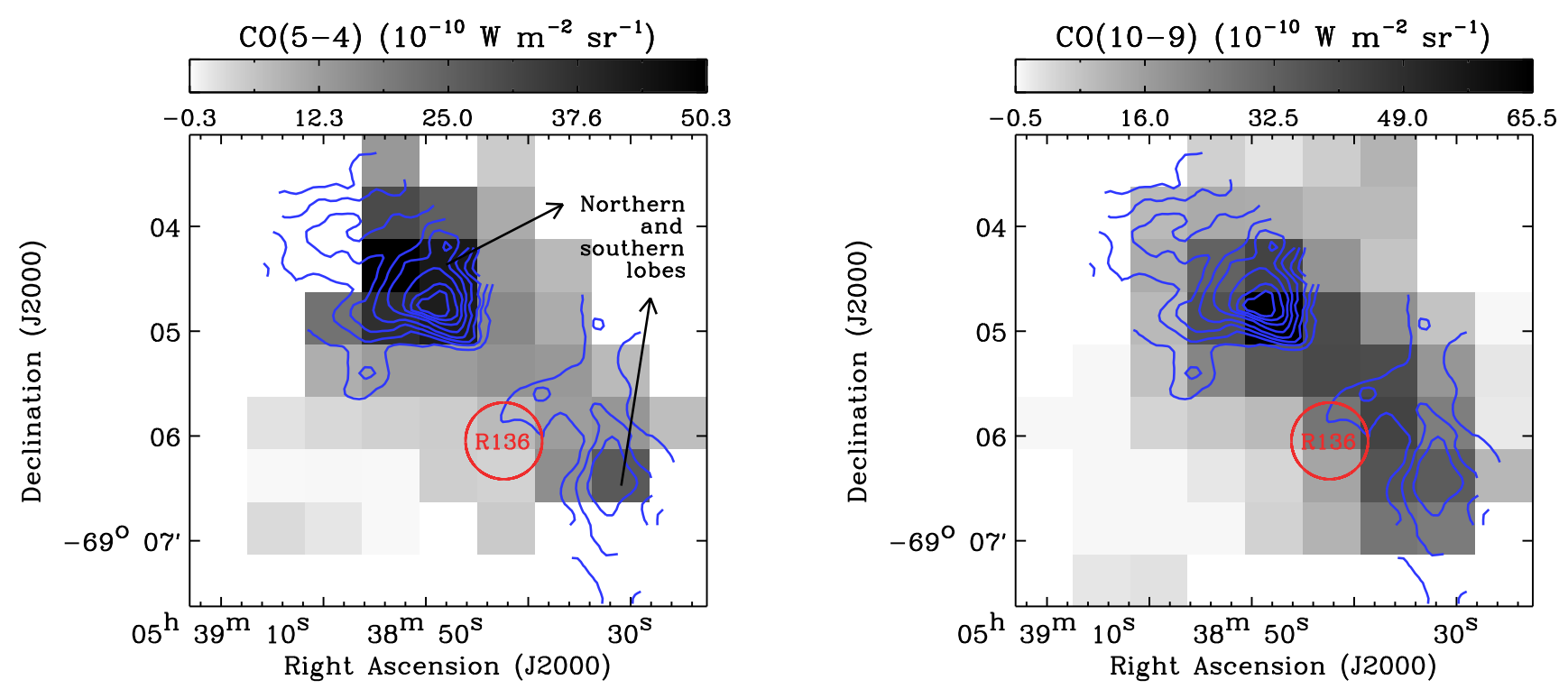

Fig. 4. Comparison between $\mathrm{CO}(J=5-4$ and $J=10-9$ on the left and right plots) and [C II] $158 \mu \mathrm{m}$ (blue contours). The PACS [C II] $158 \mu \mathrm{m}$ data at the original resolution of $12^{\prime \prime}$ are overlaid with levels ranging from 20 to $90 \%$ of the peak $\left(2.2 \times 10^{-6} \mathrm{~W} \mathrm{~m}^{-2} \mathrm{sr}^{-1}\right)$ in steps of $10 \%$. The location of the R136 cluster is indicated by the red circle. We note that the grayscale bar goes below zero simply to show pixels with low intensities.

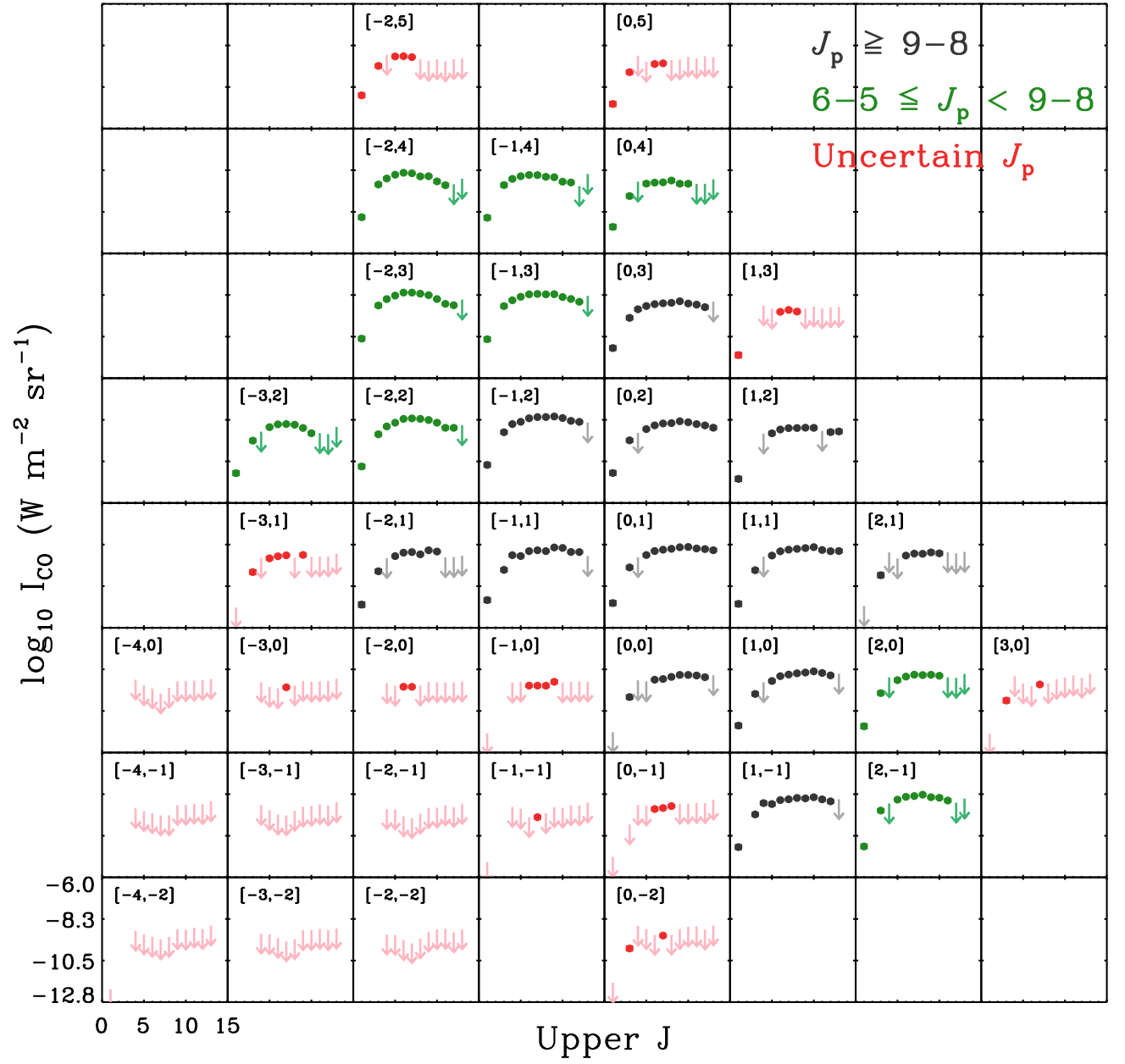

Fig. 5. CO SLEDs of 30 Doradus. Here each CO SLED was constructed using all available $\mathrm{CO}$ transitions from $J_{\mathrm{u}}=1$ to 13 for each $30^{\prime \prime}$ pixel. To indicate a location relative to the pixel that is closest to R136, a pair of numbers is shown in the top left corner of each pixel, e.g., $[0,0]$ corresponds to $(\alpha, \delta)_{\mathrm{J} 2000}=\left(05^{\mathrm{h}} 38^{\mathrm{m}} 42^{\mathrm{s}},-69^{\circ} 05^{\prime} 53^{\prime \prime}\right)$. In addition, the circles and error bars (too small to be visible in many cases) show the measured intensities and $1 \sigma$ uncertainties for detections, while the downward arrows represent the upper limits based on $S / N_{\mathrm{s}}=5$ for nondetections. Finally, the CO SLEDs are presented in different colors depending on the transition they peak $\left(J_{\mathrm{p}}\right)$ : black $\left(J_{\mathrm{p}}=9-8\right.$ or $\left.10-9\right)$, green $(6-5 \leq$ $J_{\mathrm{p}}<9-8$ ), and red (uncertain $J_{\mathrm{p}}$ due to many nondetections). The nondetections are then shown in lighter shades (gray, light green, and pink) to distinguish them from the detections. and estimated $\alpha$ on a pixel-by-pixel basis (Fig. 6 left). Twentyfive pixels were additionally masked in the process, since they have nondetections for the required transitions. We note that we did not adopt the "high- $J$ slope", $\Delta I_{\mathrm{CO}, \text { norm }}=\left[I_{\mathrm{CO}}\left(J_{\mathrm{p}}+3\right)-\right.$
$\left.I_{\mathrm{CO}}\left(J_{\mathrm{p}}\right)\right] / I_{\mathrm{CO}}\left(J_{\mathrm{p}}\right)$, the parameter Lee et al. (2016) used to characterize the observed CO SLEDs of N159W. This is because the high- $J$ slope, which measures a slope only beyond $J_{\mathrm{p}}$, was found not to capture the more general shape around the peak of 

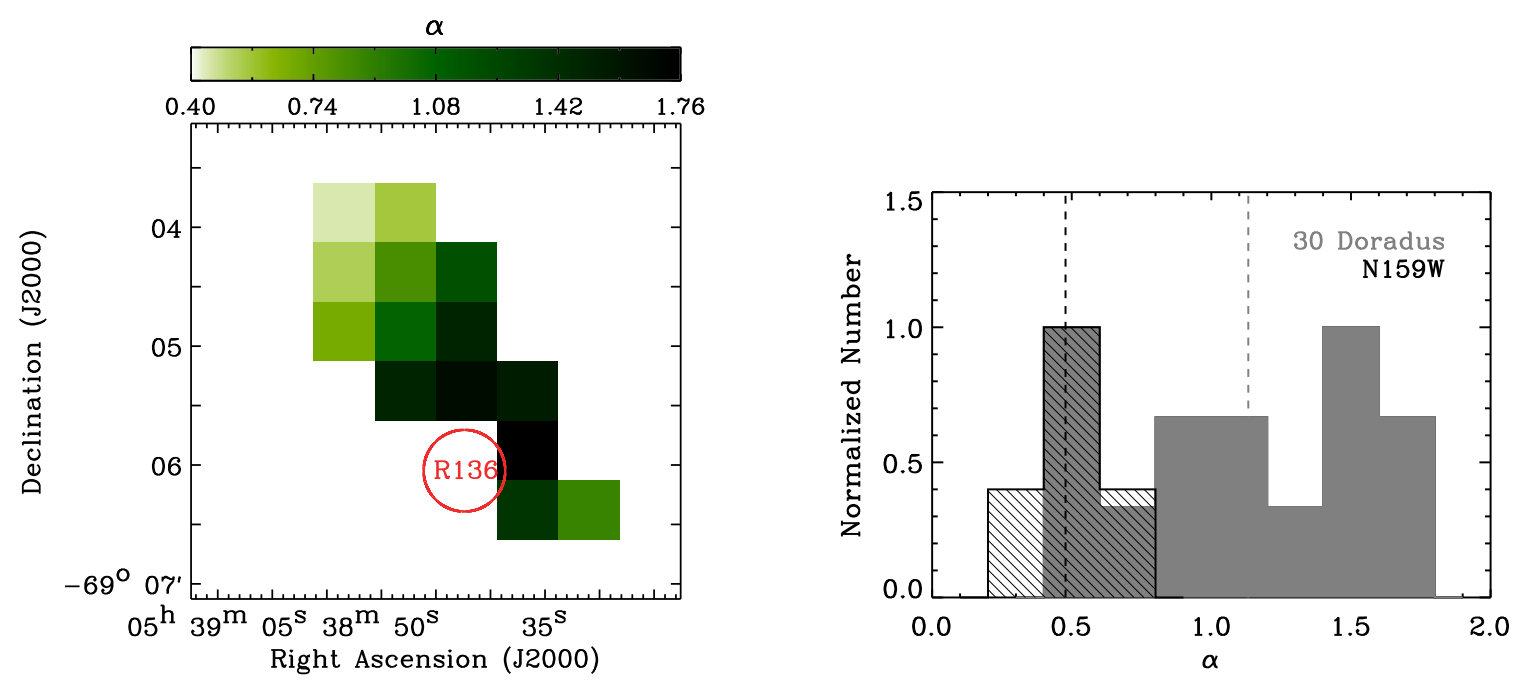

Fig. 6. Left: high-to-intermediate- $J$ CO ratio $(\alpha)$ for 30 Doradus. For the derivation of $\alpha, 25$ pixels were masked since they have nondetections for the required transitions. The location of R136 is also indicated by the red circle. Right: comparison of $\alpha$ between 30 Doradus (gray solid histogram) and N159W (black hatched histogram). The $\alpha$ values of N159W were calculated by applying Eq. (1) to the data from Lee et al. (2016), and the median value of each histogram is shown as the dashed line. For both 30 Doradus and N159W, the $\alpha$ values are on $42^{\prime \prime}$ scales.

the CO SLEDs. For example, our $[-1,4]$ and $[0,0]$ pixels would have comparable high- $J$ slopes of -0.24 despite their distinctly different CO SLEDs $([0,0]$ has a much broader peak). In addition, we note that our $\alpha$ parameter is only slightly different from what Rosenberg et al. (2015) adopted to classify 29 (U)LIRGs: we used CO $J=9-8,10-9$, and 11-10 for the high- $J$ CO contribution instead of CO $J=11-10,12-11$, and 13-12 to better reflect the properties of the CO SLEDs observed in 30 Doradus, as well as to maximize the number of available transitions for the derivation of $\alpha$.

We found that the derived $\alpha$ peaks around R136 with a value of 1.5-1.8 and decreases radially down to $\sim 0.4$, implying that the relative contribution of high- $J$ CO lines increases toward R136. Compared to $\mathrm{N} 159 \mathrm{~W}$, another massive star-forming region in the LMC, 30 Doradus shows systematically higher $\alpha$ (Fig. 6 right). Specifically, the $\alpha$ values of N159W mostly trace the lower range of the 30 Doradus histogram with a median value that is lower by a factor of two $(0.5$ versus 1.1$)$. This result indicates that the two regions have markedly different CO SLEDs. We revisit the shape of CO SLEDs as a probe of heating sources in Sect. 6.3.

The varying $\alpha$, as well as the different $J_{\mathrm{p}}$ for the individual pixels, suggest that the properties of the CO-emitting gas change across 30 Doradus on $42^{\prime \prime}$ or $10 \mathrm{pc}$ scales. For example, the peak transition and slope of the CO SLEDs depend on the gas density and temperature, while the $\mathrm{CO}$ column density affects the overall line intensities. In the following sections, the physical conditions and excitation sources of the $\mathrm{CO}$-emitting gas are examined in a self-consistent manner based on state-ofthe-art models of PDRs and shocks. In addition, the impact of high-energy photons and particles (X-rays and cosmic-rays) on $\mathrm{CO}$ emission are also assessed.

\section{Excitation sources for $\mathrm{CO}$}

\subsection{Radiative source: UV photons}

Far-UV $(E=6-13.6 \mathrm{eV})$ photons from young stars have a substantial influence on the thermal and chemical structures of the surrounding ISM. As for gas heating, the following two mechanisms are then considered important: (1) photo-electric effect on large PAH molecules and small dust grains (far-UV photons absorbed by PAH molecules and grains create free electrons, which carry off excess kinetic energy of several eVs; e.g., Bakes \& Tielens 1994; Weingartner \& Draine 2001; Weingartner et al. 2006) and (2) far-UV pumping of $\mathrm{H}_{2}$ molecules (far-UV pumped $\mathrm{H}_{2}$ molecules mostly fluoresce back to a vibrational state in the electronic ground state, and these vibrationally excited $\mathrm{H}_{2}$ molecules can heat the gas through collisional deexcitation; e.g., Sternberg \& Dalgarno 1989; Burton et al. 1990).

As the most extreme star-forming region in the Local Group of galaxies, 30 Doradus hosts numerous massive stars producing an ample amount of UV photons (Sect. 2.1). In Fig. 7, such UV sources are overlaid on the integrated intensity image of $\mathrm{CO}(9-$ 8), the transition where most of the observed CO SLEDs peak (Sect. 4.2). The strong concentration of the hot luminous stars in the central cluster R136 is particularly striking. In addition, Fig. 7 presents the UV radiation field on the plane of R136 (calculated using published catalogs of massive stars; Appendix B for details). This UV radiation field $G_{\text {stars }}$ on the plane of R136 ranges from $\sim 8 \times 10^{2}$ to $\sim 4 \times 10^{5}$ Mathis fields (its peak coincides well with R136) and can be considered as the maximum incident radiation field we would expect, since no absorption was taken into account. In the following sections, we evaluate the influence of the intense UV radiation field in 30 Doradus on $\mathrm{CO}$ emission by performing PDR modeling.

\subsubsection{Meudon PDR model}

For our purpose, we used the Meudon PDR model (Le Petit et al. 2006). This one-dimensional stationary model computes the thermal and chemical structures of a plane-parallel slab of gas and dust illuminated by a radiation field by solving radiative transfer and thermal and chemical balance. A chemical network of 157 species and 2916 reactions was adopted, and in particular $\mathrm{H}_{2}$ formation was modeled based on the prescription by Le Bourlot et al. (2012), which considers the Langmuir-Hinshelwood and Eley-Rideal mechanisms. While more sophisticated treatment of $\mathrm{H}_{2}$ formation taking into account dust temperature fluctuations is important, as demonstrated by Bron et al. (2014, 2016), we did not use this detailed model due to 

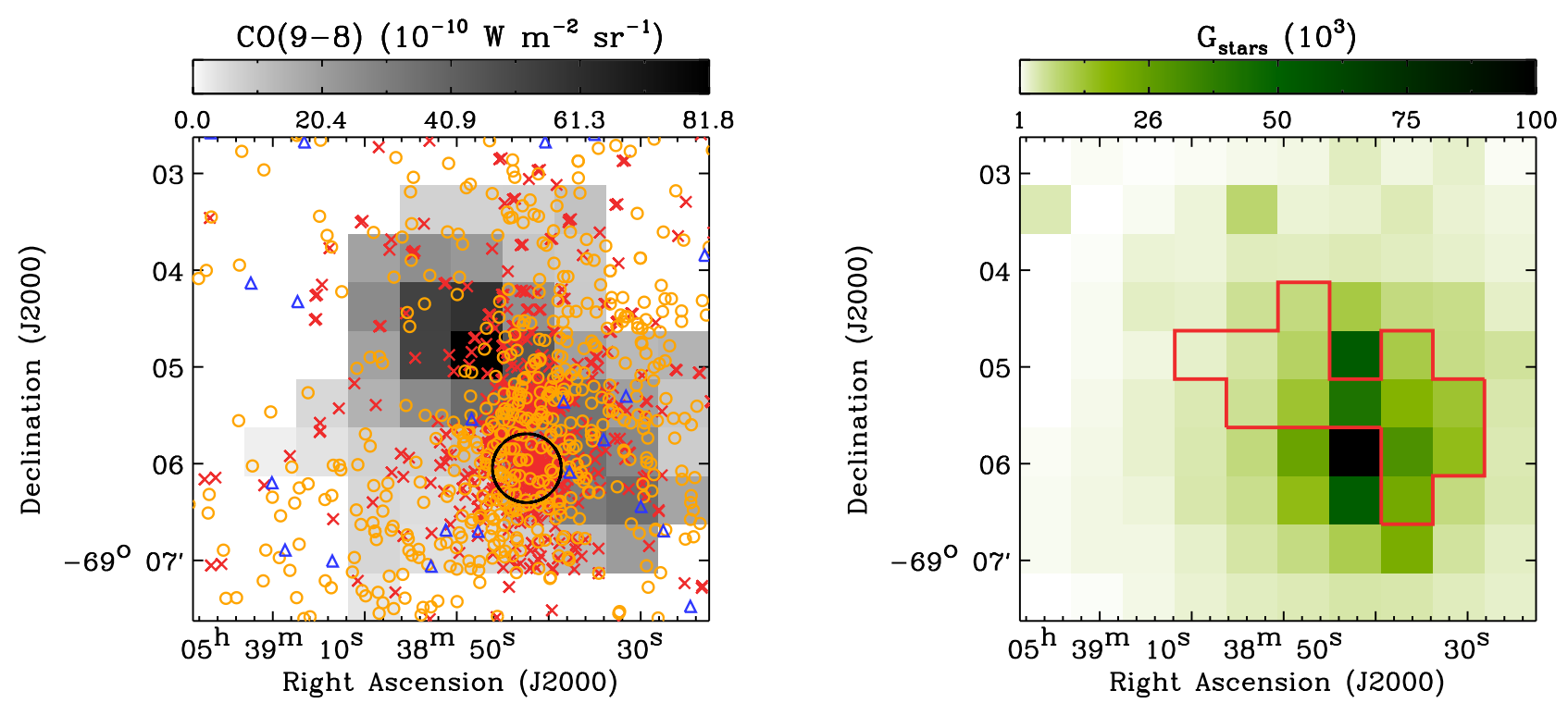

Fig. 7. Left: UV sources overlaid on the $\mathrm{CO}(9-8)$ integrated intensity image. $\sim 1.3 \times 10^{4}$ stars we used for the derivation of $G_{\text {stars }}$ (right; Appendix B for details) are presented here with different symbols depending on the stellar effective temperature $T_{\text {eff }}: T_{\text {eff }} \geq 3 \times 10^{4} \mathrm{~K}$ (1406 stars as the red crosses; mostly O-type or W-R), $10^{4} \mathrm{~K} \leq T_{\text {eff }}<3 \times 10^{4} \mathrm{~K}$ (9807 stars as the orange circles; mostly B-type) and $T_{\text {eff }}<10^{4} \mathrm{~K}$ (2116 stars as the blue triangles). The location of R136 is also indicated by the black circle. Right: stellar UV radiation field $G_{\text {stars }}$ on the plane of R136 (in units of $10^{3}$ Mathis fields). The pixels used for our PDR modeling are outlined in red.

computational time constraints. Consideration of stochastic fluctuations in the dust temperature could increase $\mathrm{H}_{2}$ formation in $\mathrm{UV}$-illuminated regions, resulting in brighter emission of $\mathrm{H}_{2}$ and other molecules that form once $\mathrm{H}_{2}$ is present (e.g., $\mathrm{CO}$ ). As for the thermal structure of the slab, the gas temperature was calculated in the stationary state considering the balance between heating and cooling. The main heating processes were the photoelectric effect on grains and $\mathrm{H}_{2}$ collisional de-excitation, and cooling rates were then derived by solving the NLTE populations of main species such as $\mathrm{C}^{+}, \mathrm{C}, \mathrm{O}, \mathrm{CO}$, and so on.

In the Meudon PDR model, the following three parameters play an important role in controlling the structure of a PDR: (1) dust extinction $A_{\mathrm{V}}$, (2) thermal pressure $P$, and (3) radiation field $G_{U V}$. Specifically, the radiation field has the shape of the interstellar radiation field in the solar neighborhood as measured by Mathis et al. (1983), and its intensity scales with the factor $G_{\mathrm{UV}}\left(G_{\mathrm{UV}}=1\right.$ corresponds to the integrated energy density of $6.0 \times 10^{-14} \mathrm{erg} \mathrm{cm}^{-3}$ for $\left.E=6-13.6 \mathrm{eV}\right)$. For our modeling of 30 Doradus, a plane-parallel slab of gas and dust with a constant $P$ and two-side illumination was considered, and a large parameter space of $A_{\mathrm{V}}=1,1.5,2,5,7,10,25,30$, 35 , and $40 \mathrm{mag}, P / k_{\mathrm{B}}=10^{4}-10^{9} \mathrm{~K} \mathrm{~cm}^{-3}$, and $G_{\mathrm{UV}}=1-10^{5}$ was examined. For two-sided illumination, the varying $G_{\mathrm{UV}}=1-10^{5}$ was incident on the front side, while the fixed $G_{\mathrm{UV}}=1$ was used for the back side. In addition, following Chevance et al. (2016), we adopted the gas phase abundances, PAH fraction $\left(f_{\mathrm{PAH}}\right)$, and dust-to-gas mass ratio $\left(M_{\text {dust }} / M_{\text {gas }}\right)$ tailored for 30 Doradus as input parameters (Table 2). Finally, the cosmic-ray ionization rate $\zeta_{\mathrm{CR}}=10^{-16} \mathrm{~s}^{-1}$ per $\mathrm{H}_{2}$ molecule was used based on observations of diffuse Galactic lines of sight (e.g., Indriolo \& McCall 2012; Indriolo et al. 2015).

\subsubsection{Strategy for PDR modeling}

The strategy for our PDR modeling was two-fold. First, we constrained $A_{\mathrm{V}}, P$, and $G_{\mathrm{UV}}$ using [C II] $158 \mu \mathrm{m}$, [C I] $370 \mu \mathrm{m}$, [O I] $145 \mu \mathrm{m}$, and FIR luminosity and assessed if the constrained
Table 2. Input parameters tailored for 30 Doradus.

\begin{tabular}{lc}
\hline \hline Parameter & Value \\
\hline Metallicity $(Z)$ & $0.5 Z_{\odot}$ \\
Dust-to-gas mass ratio $\left(M_{\text {dust }} / M_{\text {gas }}\right)$ & $5 \times 10^{-3}$ \\
PAH fraction $\left(f_{\mathrm{PAH}}\right)$ & $1 \%$ \\
\hline Element & Gas phase abundance \\
& $\left(\log _{10}(n(X) / n(\mathrm{H}))\right)$ \\
\hline $\mathrm{He}$ & -1.05 \\
$\mathrm{C}$ & -4.30 \\
$\mathrm{~N}$ & -4.91 \\
$\mathrm{O}$ & -3.75 \\
$\mathrm{Ne}$ & -4.36 \\
$\mathrm{Si}$ & -5.51 \\
$\mathrm{~S}$ & -5.32 \\
\hline
\end{tabular}

Notes. See Chevance et al. (2016) for details on these parameters.

conditions reproduce our $\mathrm{CO}$ observations. This is essentially what Lee et al. (2016) did for N159W, except that integrated intensities, rather than line ratios, were employed for our model fitting. As we show in Sect. 5.1.3 however, the PDR component responsible for the fine-structure lines and FIR luminosity turned out to produce weak $\mathrm{CO}$ emission, and we therefore further examined the conditions for $\mathrm{CO}$ emission by modeling $\mathrm{CO}$ transitions along with other observational constraints (Sect. 5.1.4). This second step was motivated by recent studies of Galactic PDRs, such as Joblin et al. (2018) for the Orion Bar and NGC 7023 NW and Wu et al. (2018) for the Carina Nebula, where CO SLEDs up to $J_{\mathrm{u}}=23$ (for the Orion Bar) were successfully reproduced by the Meudon PDR model. These studies found that high- $J \mathrm{CO}$ emission originates from the highly pressurized $\left(P / k_{\mathrm{B}} \sim 10^{8} \mathrm{~K} \mathrm{~cm}^{-3}\right)$ surface of PDRs, where hot chemistry characterized by fast ion-neutral reactions takes place 

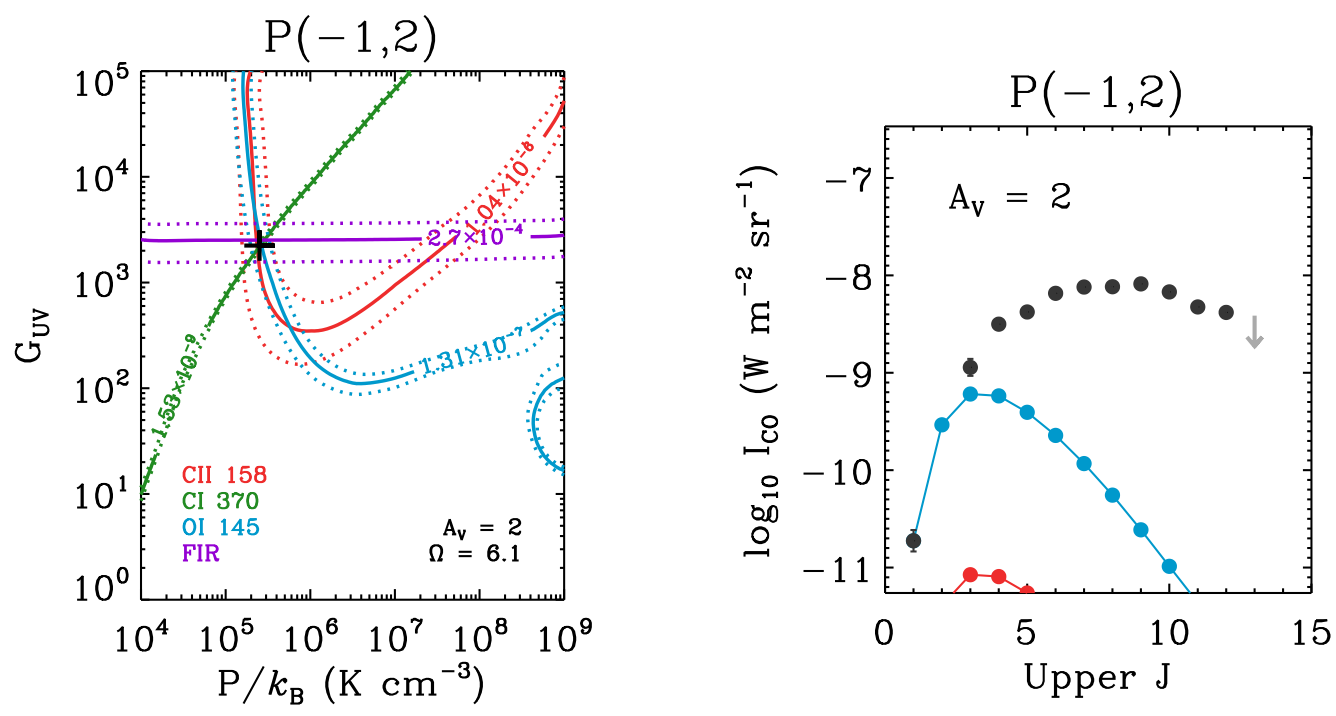

Fig. 8. Meudon PDR modeling of the three fine-structure lines and FIR luminosity. The results presented here are for the pixel [-1,2], which corresponds to $(\alpha, \delta)_{\mathrm{J} 2000}=\left(05^{\mathrm{h}} 38^{\mathrm{m}} 48^{\mathrm{s}},-69^{\circ} 04^{\prime} 53^{\prime \prime}\right)$, and the location of this pixel is indicated by the yellow star in Fig. 9. On the left plot, the observed values and their $1 \sigma$ uncertainties are shown as the solid and dotted lines ([C II] $158 \mu \mathrm{m},[\mathrm{C}$ I] $370 \mu \mathrm{m}$, [O I] $145 \mu \mathrm{m}$, and FIR luminosity in red, green, blue, and purple). The best-fit model with the minimum $\chi^{2}$ value is presented as the black cross, and the constrained $A_{\mathrm{V}}$ and $\Omega$ are summarized in the bottom right corner. The CO SLED predicted by the best-fit model (red; only partially shown since it is faint) is then compared with the observed CO SLED (dark and light gray for detections and nondetections) on the right plot. In addition, for an easier comparison, the predicted CO SLED is scaled up by a factor of 72 to match the observed $\mathrm{CO}(1-0)$ integrated intensity and shown in blue. The faint $\mathrm{CO}$ emission in the best-fit PDR model is further examined in Sect. 5.1.4.

(e.g., Goicoechea et al. 2016, 2017). Photoevaporation by strong UV radiation fields from young stars is considered to play a critical role in maintaining such high pressure at the edge of PDRs (e.g., Bron et al. 2018). In light of these new results on the physical, chemical, and dynamical processes in PDRs, we followed the approach by Joblin et al. (2018) and Wu et al. (2018) and searched for the conditions for CO by fitting $\mathrm{CO}$ lines up to $J_{\mathrm{u}}=13$. For this, we employed the most up-to-date publicly available Meudon PDR model (version 1.5.2) ${ }^{7}$, as used by Joblin et al. (2018) and Wu et al. (2018).

\subsubsection{Modeling: fine-structure lines and FIR emission}

We started PDR modeling by first examining the conditions for [C II] $158 \mu \mathrm{m}$, [C I] $370 \mu \mathrm{m}$, [O I] $145 \mu \mathrm{m}$, and FIR emission. To do so, we used the PACS and SPIRE spectroscopic data on 42" scales (Sect. 3), as well as the FIR luminosity map corrected for the contribution from the ionized medium $\left(L_{\mathrm{FIR}}^{\mathrm{PDR}}\right.$; Appendix $\mathrm{C}$ for details on the correction), and derived $\chi^{2}$ for 13 pixels where all three fine-structure lines were detected (red outlined pixels in Fig. 7 right):

$\chi^{2}=\sum_{i}\left[\frac{I_{i, \mathrm{obs}}-\left(\Omega I_{i, \mathrm{mod}}\right)}{\sigma_{i, \mathrm{obs}, \mathrm{f}}}\right]^{2}$,

where $I_{i, \mathrm{obs}}=$ observed integrated intensity, $\Omega I_{i, \bmod }=$ model prediction scaled by the beam filling factor $\Omega$, and $\sigma_{i, \text { obs, f }}=$ final $1 \sigma$ uncertainty in the observed integrated intensity. A large range of $\Omega=10^{-2}-10^{2}$ was considered in our $\chi^{2}$ calculation, and bestfit solutions were then identified as having minimum $\chi^{2}$ values. To demonstrate how our modeling was done, a plot of $G_{\mathrm{UV}}$ versus $P / k_{\mathrm{B}}$ is presented in Fig. 8 for one pixel. We note that $\Omega>$ 1 implies the presence of multiple components along a line of sight (Sect. 5.1 of Chevance et al. 2016 for more discussions).

\footnotetext{
7 https://ism.obspm.fr/
}

For 10 out of the 13 pixels, we found that best-fit PDR models with $P / k_{\mathrm{B}}=5 \times 10^{4} \sim 3 \times 10^{5} \mathrm{~K} \mathrm{~cm}^{-3}, G_{\mathrm{UV}}=400 \sim 2500$, $\Omega=2 \sim 11$, and $A_{\mathrm{V}}=1.5$ or $2 \mathrm{mag}$ reproduce well the observed fine-structure lines and FIR luminosity. These PDR solutions are presented in Fig. 9. For the other three pixels, we found that bestfit models have significantly higher $\Omega \sim 30-50$, as well as $P$ and $G_{\mathrm{UV}}$ that are not smooth across adjacent pixels. Our close examination however revealed that the observed fine-structure lines and FIR luminosity can still be reproduced within a factor of two by PDR models with $P / k_{\mathrm{B}} \sim 10^{5} \mathrm{~K} \mathrm{~cm}^{-3}, G_{\mathrm{UV}} \sim 10^{3}, \Omega \lesssim$ 10 , and $A_{\mathrm{V}}=1.5$ or 2 mag.

The images of $P$ and $G_{\mathrm{UV}}$ in Fig. 9 show that both properties peak at the north of R136 and decline outward from there. On the contrary, $\Omega$ has the minimum value of two at the regions where $P$ and $G_{\mathrm{UV}}$ peak and increases toward the outer edge of our coverage. While these spatial distributions of the PDR parameters are essentially consistent with the findings of Chevance et al. (2016), the absolute values are quite different (e.g., the maximum $P$ and $G_{\mathrm{UV}}$ values in our analysis are a factor of ten lower than those in Chevance et al. 2016). There are a number of factors that could contribute to the discrepancy, and our detailed comparison suggests that the difference in the angular resolution (42" versus $12^{\prime \prime}$ ) is most likely the primary factor (Appendix D). The same resolution effect was also noted by Chevance et al. (2016), stressing the importance of high spatial resolution in the studies of stellar radiative feedback. Finally, we note that the existence of several clouds whose individual $A_{\mathrm{V}}$ is roughly $2 \mathrm{mag}$ is indeed in agreement with what we estimated from dust SED modeling ( $A_{\mathrm{V}} \sim 8-20$ mag; Sect. 3.5), implying that the PDR component for the fine-structure lines and FIR luminosity constitutes a significant fraction $(\gtrsim 50 \%)$ of dust extinction along the observed lines of sight.

Interestingly, we found that $\mathrm{CO}$ emission is quite faint in the constrained PDR conditions. Specifically, the PDR models underestimate the observed $\mathrm{CO}$ integrated intensities by at least a factor of ten, and the discrepancy becomes greater with 

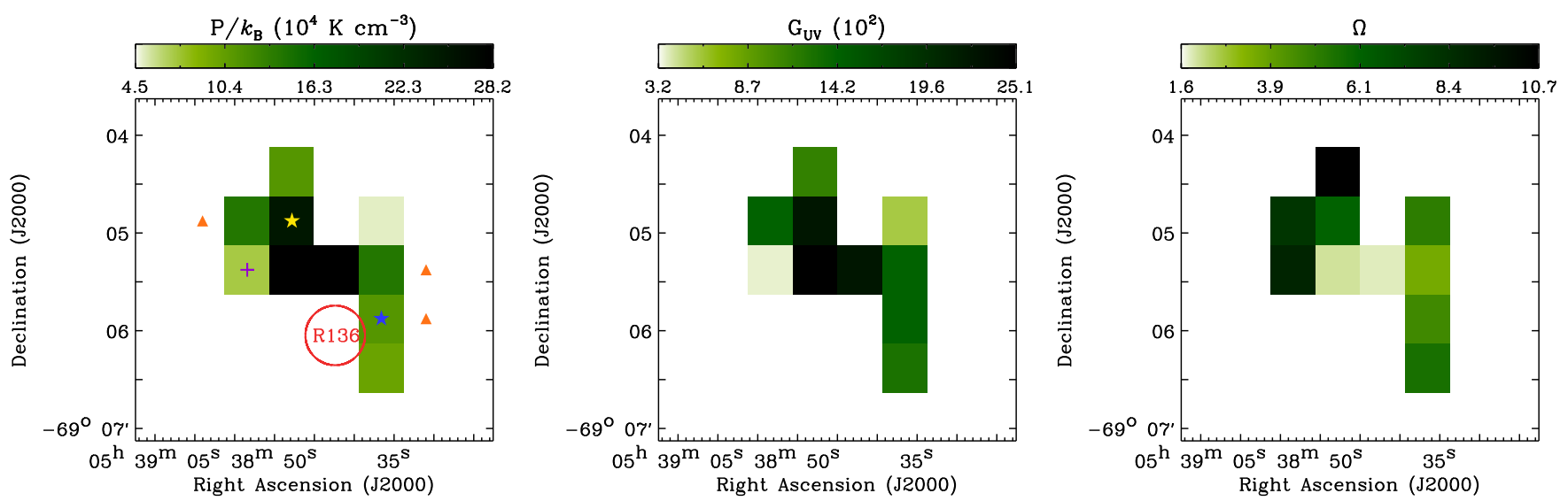

Fig. 9. Best-fit PDR solutions $\left(P / k_{\mathrm{B}}, G_{\mathrm{UV}}\right.$, and $\Omega$ on the left, middle, and right $) . A_{\mathrm{V}}=2$ mag was constrained for all but one pixel, and the location of this pixel with the smaller $A_{\mathrm{V}}=1.5 \mathrm{mag}$ is marked with the purple cross on the left panel. The three masked pixels with unreasonably high $\Omega \sim$ 30-50 are marked with the orange triangles (Sect. 5.1.3 for details), while the pixels for Figs. 8 and 10 are indicated by the yellow and blue stars, respectively. Finally, the location of R136 is shown as the red circle.

increasing $J$ (a factor of $\sim 10-70$ for $J=1-0$ to a factor of $\sim(2-5) \times 10^{5}$ for $\left.J=13-12\right)$. The worsening discrepancy with increasing $J$ suggests that the shape of the observed CO SLEDs is not reproduced by the PDR models, and we indeed found that the predicted CO SLEDs peak at $J=3-2$, which is much lower than the observed $J_{\mathrm{p}} \geq 6-5$. This large discrepancy between our $\mathrm{CO}$ observations and the model predictions (in terms of both the amplitude and shape of the CO SLEDs) is clearly demonstrated in Fig. 8. Finally, we note that $\mathrm{H}_{2} 0-0 \mathrm{~S}(3)$ is predicted to be as bright as $\sim 2 \times\left(10^{-9}-10^{-8}\right) \mathrm{W} \mathrm{m}^{-2} \mathrm{sr}^{-1}$, which is consistent with the measured upper limits based on $5 \sigma_{\mathrm{s}}$ (unfortunately, $\mathrm{H}_{2} 0-0$ $\mathrm{S}(3)$ is not detected over the 13 pixels where PDR modeling was performed).

\subsubsection{Modeling: CO lines}

Our modeling in Sect. 5.1.3 strongly suggests that CO emission in 30 Doradus arises from the conditions that are drastically different from those for the fine-structure lines and FIR luminosity $\left(P / k_{\mathrm{B}}=\mathrm{a}\right.$ few $\left(10^{4}-10^{5}\right) \mathrm{K} \mathrm{cm}^{-3}, G_{\mathrm{UV}}=\mathrm{a}$ few $\left(10^{2}-10^{3}\right)$, and $\left.A_{\mathrm{V}} \sim 2 \mathrm{mag}\right)$. More precisely, the CO-emitting regions would most likely have higher densities and/or higher temperatures (to have $J_{\mathrm{p}} \geq 6-5$ ), as well as higher dust extinction (to form more $\mathrm{CO}$ molecules, leading to brighter emission), than the [C II] $158 \mu \mathrm{m}$-emitting regions. This conclusion is essentially the same as what Lee et al. (2016) found for N159W. We then went one step further by modeling the observed $\mathrm{CO}$ transitions, examining the PDR conditions from which the CO-emitting gas would arise.

Initially, we began by computing $\chi^{2}$ using $\mathrm{CO}$ transitions up to $J=13-12$ and finding best-fit PDR models with minimum $\chi^{2}$ values. This exercise however revealed that the models become highly degenerate once high $A_{\mathrm{V}}$ ( $\left.\gtrsim 5 \mathrm{mag}\right), P / k_{\mathrm{B}}$ $\left(\gtrsim 10^{8} \mathrm{~K} \mathrm{~cm}^{-3}\right)$, and $G_{\mathrm{UV}}\left(\gtrsim 10^{3}\right)$ are achieved. In addition, many best-fit models were incompatible with the observed finestructure lines and FIR luminosity. Specifically, the best-fit models always underestimate [C II] $158 \mu \mathrm{m}$ and FIR luminosity (model-to-observation ratio $\lesssim 0.1$ ), while mostly reproducing [O I] $145 \mu \mathrm{m}$ and [C I] $370 \mu \mathrm{m}$ within a factor of four or less. As for $\mathrm{H}_{2}$ 0-0 S(3), the best-fit models predict overly bright emission in many cases. This result suggests that at least two components, the low- $P$ and high- $P$ PDRs, would be required to explain all the transitions we observed in 30 Doradus. To work around the degeneracy issue and exclude models with unreasonable predictions for the fine-structure lines and FIR luminosity, we then decided to evaluate a collection of PDR models that reproduce the observed $\mathrm{CO}$ reasonably well, rather than focusing on best-fit models, and employed other observations as secondary constraints. To this end, we selected the PDR models that satisfy the following criteria: (1) the detected $\mathrm{CO}$ lines are reproduced within a factor of two. In the case of $\mathrm{CO}(1-0)$, the prediction is only required to not exceed twice the observed value, considering that $\mathrm{CO}(1-0)$ could trace physical conditions that are different from those traced by intermediateand high- $J$ CO lines (e.g., Joblin et al. 2018; Wu et al. 2018). (2) The model predictions agree with the measured upper limits when the CO lines are not detected. (3) For [C II] $158 \mu \mathrm{m}$, [C I] $370 \mu \mathrm{m}$, [O I] $145 \mu \mathrm{m}$, and FIR luminosity, the model predictions plus the contributions from the low- $P$ PDR component in Sect. 5.1.3 are within a factor of two from the observed values. (4) Finally, the model prediction plus the contribution from the low- $P$ PDR component should be consistent with the $\mathrm{H}_{2}$ $0-0 \mathrm{~S}(3)$ upper limit. Along with a large range of $\Omega=10^{-4}-1^{8}$, these four criteria were applied to the 10 pixels where we constrained the best-fit PDR models for the fine-structure lines and FIR luminosity (Fig. 9). Since bright $\mathrm{CO}(J \gtrsim 4-3)$ emission mostly arises from a relatively narrow range of physical conditions $\left(A_{\mathrm{V}} \gtrsim 5 \mathrm{mag}, P / k_{\mathrm{B}} \gtrsim 10^{8} \mathrm{~K} \mathrm{~cm}^{-3}\right.$, and $\left.G_{\mathrm{UV}} \gtrsim 10^{3}\right)$ in the Meudon PDR model, slight changes in modeling, such as removing the (3) and (4) criteria or modeling CO lines with $J \gtrsim 4-3$ only, do not have a large impact on the constrained parameters. Finally, we note that our modeling with two components of gas is simplistic, given that multiple components would likely be mixed on $\sim 10$ pc scales. Nevertheless, our analyses would still provide average physical conditions of the components within the beam.

Overall, we were able to find reasonably good PDR solutions that meet the above selection criteria for 8 out of the 10 pixels $([-1,1]$ and $[1,2]$ do not have solutions). The constrained parameters were then as follows: $A_{\mathrm{V}}=5-40 \mathrm{mag}, P / k_{\mathrm{B}}=\sim 10^{8}$ $10^{9} \mathrm{~K} \mathrm{~cm}^{-3}, G_{\mathrm{UV}}=\sim 10^{3}-10^{5}$, and $\Omega=\sim 0.01-0.1$. We note that $A_{\mathrm{V}}$ was not well constrained, since increasing $A_{\mathrm{V}}$ beyond $5 \mathrm{mag}$ only increases the size of the cold CO core $(<50 \mathrm{~K})$ in a PDR, and not the warm layer $(z 50-100 \mathrm{~K})$ where most of intermediate-

\footnotetext{
8 For $\mathrm{CO}$ emission, we examined beam filling factors that are smaller than those in Sect. 5.1.3, primarily based on the ALMA CO(2-1) observations by Indebetouw et al. (2013) (where CO clumps in 30 Doradus were found to be much smaller than our $30^{\prime \prime}$ pixels).
} 

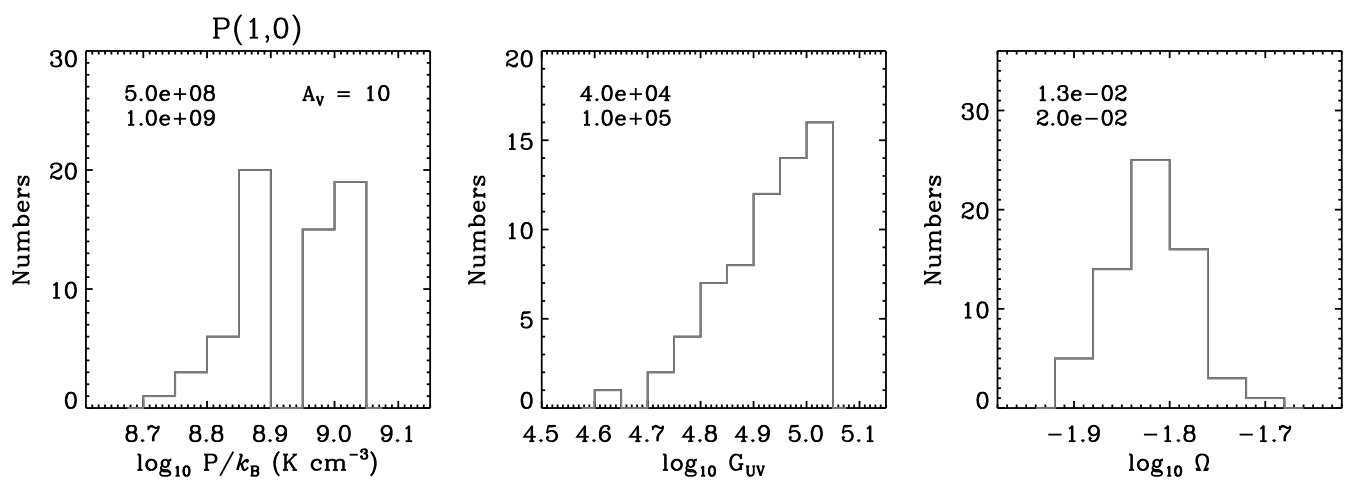

Fig. 10. PDR models with $A_{\mathrm{V}}=10$ mag that were selected by the criteria in Sect. $5.1 .4\left(P / k_{\mathrm{B}}, G_{\mathrm{UV}}\right.$, and $\Omega$ on the left, middle, and right panels; number of selected models $=64)$. This particular example is for the pixel $[1,0]$ which corresponds to $(\alpha, \delta)_{\mathrm{J} 2000}=\left(05^{\mathrm{h}} 38^{\mathrm{m}} 37^{\mathrm{s}},-69^{\circ} 05^{\prime} 53^{\prime \prime}\right)$, and the location of the pixel is indicated by the blue star in Fig. 9. In each plot, the minimum and maximum values of the PDR parameter are shown in the top left corner.
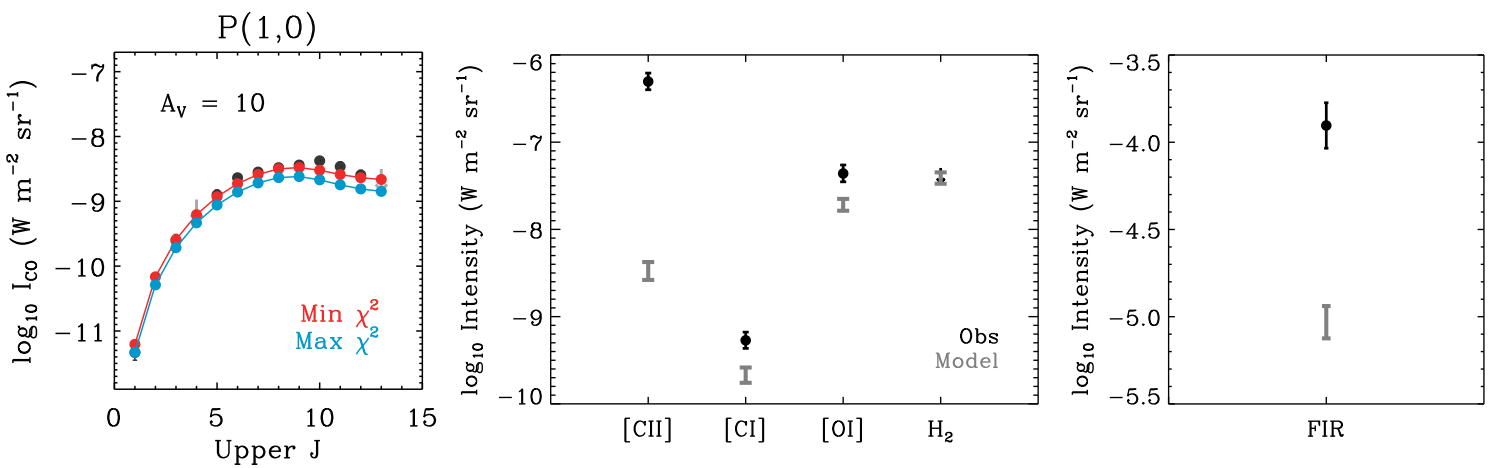

Fig. 11. Comparison between the observations and the predictions from the PDR models in Fig. 10. In the left plot, the observed CO SLED (dark and light gray for detections and nondetections) is compared with the predictions from two models (those resulting in minimum and maximum $\chi^{2}$ values with respect to the observed CO lines are presented in red and blue). In the middle and right plots, the observed [C II] $158 \mu \mathrm{m},[\mathrm{C} \mathrm{I}] 370 \mu \mathrm{m}$, [O I] $145 \mu \mathrm{m}, \mathrm{H}_{2} 0-0 \mathrm{~S}(3)$, and FIR luminosity are shown (black) along with the ranges of the model predictions (from minimum to maximum values; gray).

and high- $J$ CO emission originates (Sect. 6.1). In addition, $\Omega \sim 0.01-0.1$ implies that the CO-emitting clumps would be $\sim 0.7-2$ pc in size, which is consistent with the ALMA CO(2-1) observations where $\mathrm{CO}$ emission was found to primarily arise from structures of $\sim 0.3-1 \mathrm{pc}$ in size (Indebetouw et al. 2013). As an example, we present the selected PDR models and predicted intensities for one pixel in Figs. 10 and 11.

Interestingly, we found that the constrained PDR models significantly underestimate [C II] $158 \mu \mathrm{m}$ and FIR luminosity (e.g., Fig. 11): the discrepancy with our data ranges from $\sim 100$ to $\sim 10^{3}$ for [C II] $158 \mu \mathrm{m}$ and from $\sim 10$ to $\sim 100$ for FIR luminosity. On the other hand, [O I] $145 \mu \mathrm{m}$ and [C I] $370 \mu \mathrm{m}$ were marginally reproduced (within a factor of four or less) in most cases: 4 out of the 8 pixels for [O I] $145 \mu \mathrm{m}$ and 7 out of the 8 pixels for [C I] $370 \mu \mathrm{m}$. The measured $\mathrm{H}_{2} 0-0 \mathrm{~S}(3)$ upper limits were also consistent with the model predictions. All in all, these results indicate that at least two PDR components are needed to explain all the observational constraints we have for 30 Doradus: (1) the low- $P\left(10^{4}-10^{5} \mathrm{~K} \mathrm{~cm}^{-3}\right)$ component that provides most of the dust extinction along the observed lines of sight and emits intensely in [C II] $158 \mu \mathrm{m}$ and FIR continuum and (2) the high- $P$ $\left(10^{8}-10^{9} \mathrm{~K} \mathrm{~cm}^{-3}\right)$ component that is mainly responsible for $\mathrm{CO}$ emission. For [O I] $145 \mu \mathrm{m}$, [C I] $370 \mu \mathrm{m}$, and $\mathrm{H}_{2} 0-0 \mathrm{~S}(3)$, both components contribute. We indeed confirmed that the sum of the two components fully reproduces the observations in our study (including $\mathrm{CO} J=1-0$ ).
To understand how different the two components are in terms of their physical properties, we then made a comparison between the constrained PDR parameters on a pixel-by-pixel basis (Fig. 12). Our comparison revealed first of all that the high$P$ component indeed has significantly higher $P$ than the low- $P$ component (a factor of $\sim 10^{3}-10^{4}$ ). Combined with the fact that the high- $P$ models have much smaller $\Omega$ than the low- $P$ models (a factor of $\sim 10^{2}-10^{3}$ ), this result implies that the CO-emitting regions in 30 Doradus are more compact, as well as warmer and/or denser, than the [C II]-emitting regions. The relative distribution of the two regions can subsequently be inferred from the $G_{\mathrm{UV}}$ values. For most of the pixels in our consideration, we found that the UV radiation incident onto the surface of the CO-emitting regions is more intense than that for the [C II]emitting regions (by up to a factor of $~ 300$ ). These pixels also have $G_{\text {stars }}$ that is comparable to or slightly higher than $G_{\mathrm{UV}}$ for the high- $P$ component. Considering that the UV radiation field would be most intense on the plane of R136 $\left(G_{\text {stars }}\right)$ and decrease as the distance $r$ from R136 increases $\left(\propto 1 / r^{2}\right.$ if no absorption is taken into account), our results imply that the CO-emitting regions would likely be either in between R136 and the [C II]emitting regions or much closer to $\mathrm{R} 136$. For the pixel $[1,1]$ however, $G_{\mathrm{UV}}$ for the high- $P$ component is higher than $G_{\text {stars }}$ by up to a factor of five, a somewhat large discrepancy even considering uncertainties in $G_{\mathrm{UV}}$ and $G_{\text {stars }}$ (meaning that the PDR solution could be unreasonable). 
M.-Y. Lee et al.: Radiative and mechanical feedback into the molecular gas in the Large Magellanic Cloud. II. 30 Doradus
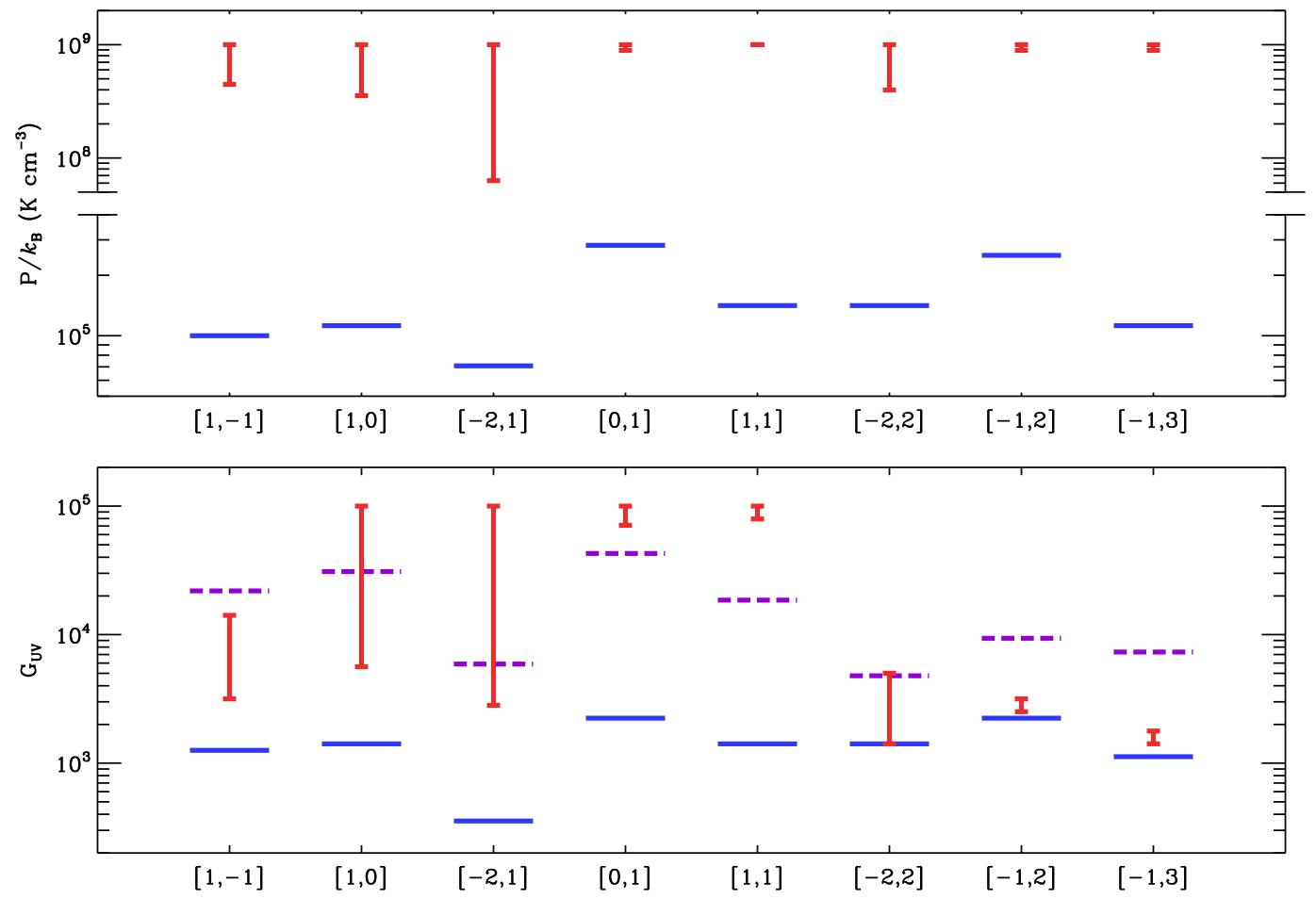

Fig. 12. Comparison between the low- and high- $P$ PDR models $\left(P / k_{\mathrm{B}}, G_{\mathrm{Uv}}\right.$, and $\Omega$ on the top, middle, and bottom panels). In each plot, the low- $P$ models we constrained using the finestructure lines and FIR luminosity (Sect. 5.1.3) are indicated by the blue solid lines, while the high- $P$ models for the $\mathrm{CO}$ lines are presented as the red bars (ranging from the minimum to maximum values). In the $G_{\mathrm{UV}}$ plot, $G_{\text {stars }}$ in Fig. 7 is also shown as the purple dashed lines. In total, eight pixels where we found reasonably good high- $P$ models are shown in each plot, and the location of each pixel can be inferred from Figs. 3 and 5. Finally, we note that the $P$ and $\Omega$ plots have broken $y$-axes to show a wide range of the data.

In summary, we conclude that the observed $\mathrm{CO}$ transitions in 30 Doradus (up to $J=13-12$ ) could be powered by UV photons and likely originate from highly compressed $\left(P / k_{\mathrm{B}} \sim 10^{8}-\right.$ $\left.10^{9} \mathrm{~K} \mathrm{~cm}^{-3}\right)$, highly illuminated $\left(G_{\mathrm{UV}} \sim 10^{3}-10^{5}\right)$ clumps with a scale of $\sim 0.7-2 \mathrm{pc}$. These clumps are also partially responsible for the observed [C I] $370 \mu \mathrm{m}$ and [O I] $145 \mu \mathrm{m}$, but emit quite faintly in [C II] $158 \mu \mathrm{m}$ and FIR continuum emission, hinting at the presence of another component with drastically different physical properties. Our PDR modeling then suggests that this additional component indeed has lower $P$ (a few $\left.\left(10^{4}-10^{5}\right) \mathrm{K} \mathrm{cm}^{-3}\right)$ and $G_{\mathrm{UV}}\left(\mathrm{a}\right.$ few $\left.\left(10^{2}-10^{3}\right)\right)$ and likely fills a large fraction of our $30^{\prime \prime}$ pixels. Interestingly, the constrained PDR parameters imply that the two distinct components are likely not co-spatial (the high- $P$ PDR component closer to R136), which is a somewhat unusual geometry. More detailed properties of the two PDR components (e.g., density and temperature) are discussed in Sect. 6.1, along with another viable heating source for $\mathrm{CO}$ : shocks.

\subsection{Radiative source: $X$-rays and cosmic-rays}

As described in Sect. 2.3, abundant X-rays and cosmic-rays exist in 30 Doradus. These high-energy photons and particles can play an important role in gas heating (mainly through photoionization of atoms and molecules), and yet we evaluated that their impact on the observed $\mathrm{CO}$ lines is negligible. Our evaluation was based on Lee et al. (2016) and can be summarized as follows.

In Lee et al. (2016), we examined the influence of X-rays by considering the most luminous X-ray source in the LMC, LMC X-1 (a black hole binary). The maximum incident X-ray flux of $10^{-2} \mathrm{erg} \mathrm{s}^{-1} \mathrm{~cm}^{-2}$ (maximum since absorption between LMC X-1 and N159W was not taken into account) was incorporated into PDR modeling, and we found that X-rays lead to only a factor of three or so change in the total CO integrated intensity. Considering that the X-ray flux in 30 Doradus is much lower (up to $10^{-4} \mathrm{erg} \mathrm{s}^{-1} \mathrm{~cm}^{-2}$ only) than the maximum case for N159W, we then concluded that X-rays most likely provide only a minor contribution to $\mathrm{CO}$ heating in 30 Doradus.

As for cosmic-ray heating, we again followed the simple calculation by Lee et al. (2016). In this calculation, $\mathrm{H}_{2}$ cooling (primary cooling process for the warm and dense medium; e.g., Le Bourlot et al. 1999) was equated with cosmic-ray heating to estimate the cosmic-ray ionization rate of $\zeta_{\mathrm{CR}} \gtrsim 3 \times 10^{-13} \mathrm{~s}^{-1}$ that is required to fully explain the warm CO in N159W. While this cosmic-ray ionization rate is higher than the typical value for the diffuse ISM in the solar neighborhood by more than a factor of 1000 (e.g., Indriolo et al. 2015), the measured $\gamma$-ray emissivity of N159W ( $\sim 10^{26}$ photons s ${ }^{-1} \mathrm{sr}^{-1}$ per hydrogen atom) is comparable to the local ISM value (e.g., Abdo et al. 2009), suggesting that the cosmic-ray density in N159W is not atypical. Similarly, 
considering that the CO-emitting gas in 30 Doradus is warm and dense as in N159W (Sect. 6.1 for details), yet the $\gamma$-ray emissivity is only $\sim 3 \times 10^{26}$ photons $\mathrm{s}^{-1} \mathrm{sr}^{-1}$ per hydrogen atom (Abdo et al. 2010), it is again likely that cosmic-rays in 30 Doradus are not abundant enough for $\mathrm{CO}$ heating.

\subsection{Mechanical source: shocks}

Shocks are ubiquitous in the ISM, being continuously driven by various energetic processes. These processes include stellar activities such as outflows (YSOs and red giant stars), winds (OB-type and $\mathrm{W}-\mathrm{R}$ stars), and explosions (novae and $\mathrm{SNe}$ ), as well as nonstellar activities such as colliding clouds and spiral density waves (e.g., Hollenbach et al. 1989). Shocks can be an important source of heating, since they effectively transform the bulk of the injected mechanical energy into thermal energy. In the particular case of the dense and magnetized medium with a low fractional ionization (essentially corresponding to star-forming regions such as 30 Doradus), C-type shocks can develop, whose main characteristics include the following: (1) molecules are accelerated without being thermally dissociated; and (2) the shocked medium radiates primarily in rotationvibration transitions of molecules, as well as fine-structure lines of atoms and ions (Draine \& McKee 1993). The emission from C-type shocks largely appears at IR wavelengths and provides a powerful means to probe the physical properties of the shocks and the ambient medium.

\subsubsection{Paris-Durham shock model}

Motivated by the results from Lee et al. (2016) for N159W, we evaluated whether low-velocity C-type shocks could be another important source of heating for $\mathrm{CO}$ in 30 Doradus by comparing the observed line emission with predictions from the ParisDurham shock model ${ }^{7}$ (Flower \& Pineau des Forêts 2015). This one-dimensional stationary model simulates the propagation of a shock wave ( $\mathrm{J}$ - or $\mathrm{C}$-type) through a layer of gas and dust and calculates the physical, chemical, and dynamical properties of the shocked layer. For our analysis, we used the modified version by Lesaffre et al. (2013) to model UV-irradiated shocks and created a grid of models with the following parameters: (1) preshock density $n_{\text {pre }}=(1,2$, and 5$) \times 10^{4},(1,2$, and 5$) \times 10^{5}$, and $10^{6} \mathrm{~cm}^{-3}$; (2) shock velocity $v_{\mathrm{s}}=4-11 \mathrm{~km} \mathrm{~s}^{-1}$ with $0.5 \mathrm{~km} \mathrm{~s}^{-1}$ steps and 12-30 $\mathrm{km} \mathrm{s}^{-1}$ with $2 \mathrm{~km} \mathrm{~s}^{-1}$ steps (a finer grid was produced for $v_{\mathrm{s}}=4-11 \mathrm{~km} \mathrm{~s}^{-1}$ to properly sample a variation in $\mathrm{H}_{2} 0-0 \mathrm{~S}(3)$ of a factor of $\sim 100$ over this velocity range); (3) UV radiation field $G_{\mathrm{UV}}^{\prime}$ (defined as a scaling factor relative to the Draine 1978 radiation field $)=0$ and 1 ; (4) dimensionless magnetic field parameter $b$ (defined as $(B / \mu \mathrm{G}) /\left(n_{\text {pre }} / \mathrm{cm}^{-3}\right)^{1 / 2}$, where $B$ is the strength of the magnetic field transverse to the direction of shock propagation) $=1$; and (5) same gas and dust properties as used in our PDR modeling (Table 2 for details). In our grid of models, the magnetosonic speed varies from $\sim 20 \mathrm{~km} \mathrm{~s}^{-1}$ $\left(G_{\mathrm{UV}}^{\prime}=1\right.$ case $)$ to $\sim 80 \mathrm{~km} \mathrm{~s}^{-1}\left(G_{\mathrm{UV}}^{\prime}=0\right.$ case $)$, and the postshock pressure (roughly determined by the ram-pressure of the pre-shock medium) has a range of $\sim 10^{5}-10^{9} \mathrm{~K} \mathrm{~cm}^{-3}$. All our models fall into the C-type shock category. Finally, the calculated abundances of atoms and molecules were post-processed via the LVG method by Gusdorf et al. $(2012,2015)$ to compute level populations, line emissivities, and integrated intensities.

\subsubsection{Strategy for shock modeling}

To examine the properties of shocks that could possibly heat $\mathrm{CO}$ in 30 Doradus, we went one step further than Lee et al. (2016) by fitting the observed $\mathrm{CO}$ transitions with the shock models. In an attempt to break the degeneracy between the model parameters, we then considered other constraints in our shock modeling, such as $\mathrm{H}_{2} \mathrm{O}-0 \mathrm{~S}(3)$ and [C I] $370 \mu \mathrm{m}$.

\subsubsection{Modeling: CO lines}

We began shock modeling by first deriving $\chi^{2}$ using the observed $\mathrm{CO}(J=3-2$ to $13-12)$ and $\mathrm{H}_{2} 0-0 \mathrm{~S}(3)$ transitions for 23 pixels that contain more than five $\mathrm{CO}$ detections (so that the number of constraints $\geq 1+$ the number of model parameters, $n_{\mathrm{pre}}, v_{\mathrm{s}}, G_{\mathrm{UV}}^{\prime}$, and $\Omega$ ). Our $\chi^{2}$ calculation was essentially based on Eq. (2), but with an additional consideration for nondetections. Specifically, we set $\left[I_{i, \mathrm{obs}}-\left(\Omega I_{i, \text { mod }}\right)\right] / \sigma_{i, \mathrm{obs}, \mathrm{f}}=0$ for the transitions whose $5 \sigma_{\mathrm{s}}$-based upper limits are consistent with model predictions. When the model predictions were higher than the upper limits, we then simply excluded such bad models from our analysis (meaning that the nondetections were used to provide hard limits on the models). In our $\chi^{2}$ analysis, $\mathrm{CO}(1-0)$ was not included to consider a possible presence of some cold pre-shock gas that could emit brightly in $\mathrm{CO}(1-0)$ (e.g., Lee et al. 2016). Finally, the same $\Omega=10^{-4}-1$ as used in our PDR modeling (Sect. 5.1.4) was examined.

The inclusion of $\mathrm{H}_{2} \quad 0-0 \mathrm{~S}(3)$ in our $\chi^{2}$ analysis was intended to mitigate the degeneracy between $n_{\text {pre }}$ and $v_{\mathrm{s}}$. In particular, we found that $\mathrm{H}_{2} \mathrm{O}-0 \mathrm{~S}(3)$, even with upper limits, can effectively differentiate high-density $\left(>10^{4} \mathrm{~cm}^{-3}\right)$, lowvelocity $\left(\lesssim 10 \mathrm{~km} \mathrm{~s}^{-1}\right)$ shocks from low-density $\left(\sim 10^{4} \mathrm{~cm}^{-3}\right)$, high-velocity $\left(\gtrsim 20 \mathrm{~km} \mathrm{~s}^{-1}\right)$ shocks. To demonstrate this, we show the observed $\mathrm{CO}$ and $\mathrm{H}_{2} \quad 0-0 \mathrm{~S}(3)$ for the pixel $[-1,2]$ in Fig. 13, along with three different shock models $\left(n_{\text {pre }}=10^{4}, 5 \times 10^{4}\right.$, and $10^{6} \mathrm{~cm}^{-3} ; v_{\mathrm{s}}=28,7.5$, and $4 \mathrm{~km} \mathrm{~s}^{-1}$; $\left.G_{\mathrm{UV}}^{\prime}=0 ; \Omega \sim 0.1\right)$. These shock models all reproduce the observed CO SLED within a factor of two, while showing a factor of $\sim 200$ difference in $\mathrm{H}_{2} \quad 0-0 \mathrm{~S}(3)$. Specifically, the highest-velocity shock produces the brightest $\mathrm{H}_{2} 0-0 \mathrm{~S}(3)$ of $\sim 4 \times 10^{-7} \mathrm{~W} \mathrm{~m}^{-2} \mathrm{sr}^{-1}$ (primarily due to the high temperature of $\sim 10^{3} \mathrm{~K}$ that is achieved by strong compression), and the measured upper limit clearly rules out this model. On the other hand, the other two models have relatively low temperatures of $\sim 10^{2} \mathrm{~K}$ and show an insignificant difference in $\mathrm{H}_{2} 0-0 \mathrm{~S}(3)$ emission (a factor of four). Our $\mathrm{H}_{2}$ observations are unfortunately not sensitive enough to discriminate this level of minor difference (e.g., only 2 out of the 23 pixels have detections with $\left.S / N_{\mathrm{s}} \sim 5\right)$, resulting in the degeneracy in $5 \times 10^{4} \mathrm{~cm}^{-3} \lesssim$ $n_{\text {pre }} \lesssim 10^{6} \mathrm{~cm}^{-3}$ and $v_{\mathrm{s}} \lesssim 10 \mathrm{~km} \mathrm{~s}^{-1}$ in our shock analysis.

In addition to $n_{\text {pre }}$ and $v_{\mathrm{s}}, G_{\mathrm{UV}}^{\prime}$ and $\Omega$ are also degenerate in the shock models $\left(G_{\mathrm{UV}}^{\prime}=1\right.$ would dissociate more $\mathrm{CO}$ molecules than the $G_{\mathrm{UV}}^{\prime}=0$ case, requiring a larger beam filling factor to achieve the same level of CO emission), and we tried to mitigate this degeneracy by considering the observed [C I] $370 \mu \mathrm{m}$ emission. For example, 8 out of the 23 pixels in our shock analysis have best-fit models with $G_{\mathrm{UV}}^{\prime}=1$, and these $G_{\mathrm{UV}}^{\prime}=1$ models overpredict [C I] $370 \mu \mathrm{m}$ by a factor of $\sim 10-20^{9}$. In addition, the constrained $\Omega$ for these models is close to one, which is not compatible with what the high-resolution ALMA $\mathrm{CO}(2-1)$ observations suggest $(\Omega \lesssim 0.1)$. Considering that this is indeed a general trend (shock models with $G_{\mathrm{UV}}^{\prime}=1$ that reproduce our $\mathrm{CO}$ and $\mathrm{H}_{2} \quad 0-0 \mathrm{~S}(3)$ observations tend to overpredict [C I] $370 \mu \mathrm{m}$ with unreasonably large beam filling factors of $\sim 1$ ), we then determined final shock properties by selecting $G_{\mathrm{UV}}^{\prime}=0$ models with minimum $\chi^{2}$ values and present them in Fig. 14.

$\overline{9}[\mathrm{C}$ II $] 158$ and [O I] $145 \mu \mathrm{m}$ in these models are still much fainter than those in our observations. 

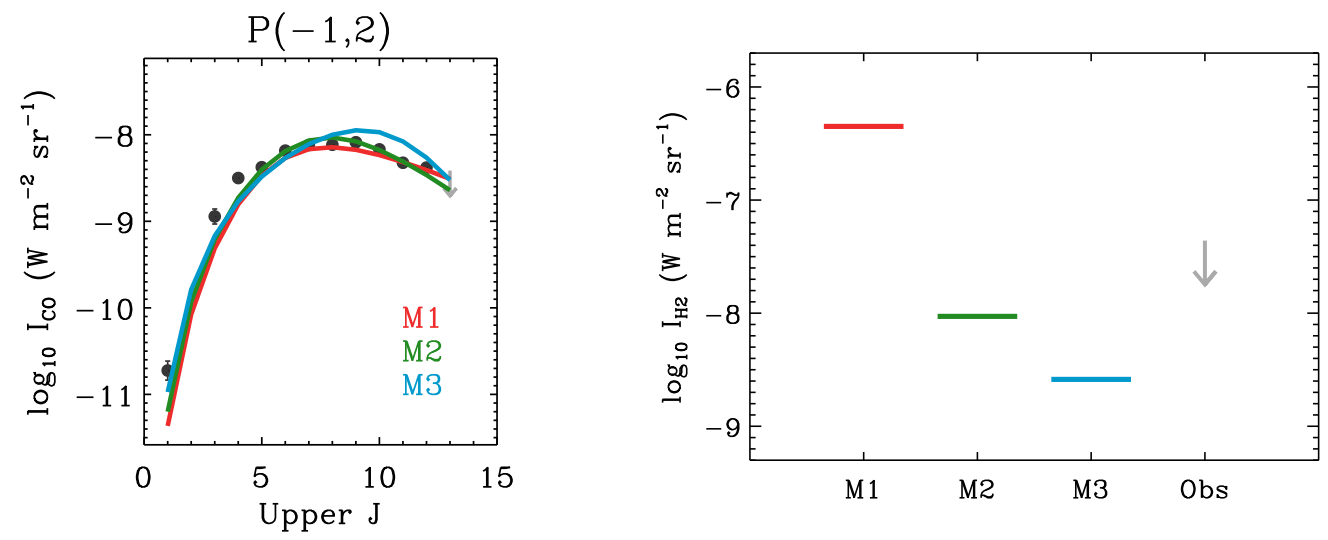

Fig. 13. Degeneracy in $n_{\text {pre }}$ and $v_{\mathrm{s}}$. To illustrate this issue, the observed $\mathrm{CO}$ and $\mathrm{H}_{2} 0-0 \mathrm{~S}(3)$ transitions of the pixel $[-1,2]$ are shown in the left and right plots along with three different shock models ("M1" in red: $n_{\mathrm{pre}}=10^{4} \mathrm{~cm}^{-3}$ and $v_{\mathrm{s}}=28 \mathrm{~km} \mathrm{~s}^{-1}$; "M2" in green: $n_{\text {pre }}=5 \times 10^{4} \mathrm{~cm}^{-3}$ and $v_{\mathrm{s}}=7.5 \mathrm{~km} \mathrm{~s}^{-1}$; "M3" in blue: $n_{\text {pre }}=10^{6} \mathrm{~cm}^{-3}$ and $v_{\mathrm{s}}=4 \mathrm{~km} \mathrm{~s}^{-1}$ ). For both plots, dark and light gray colors are used to represent detections and nondetections, and $G_{\mathrm{UV}}^{\prime}=0$ and $\Omega \sim 0.1$ are adopted for the three shock models.

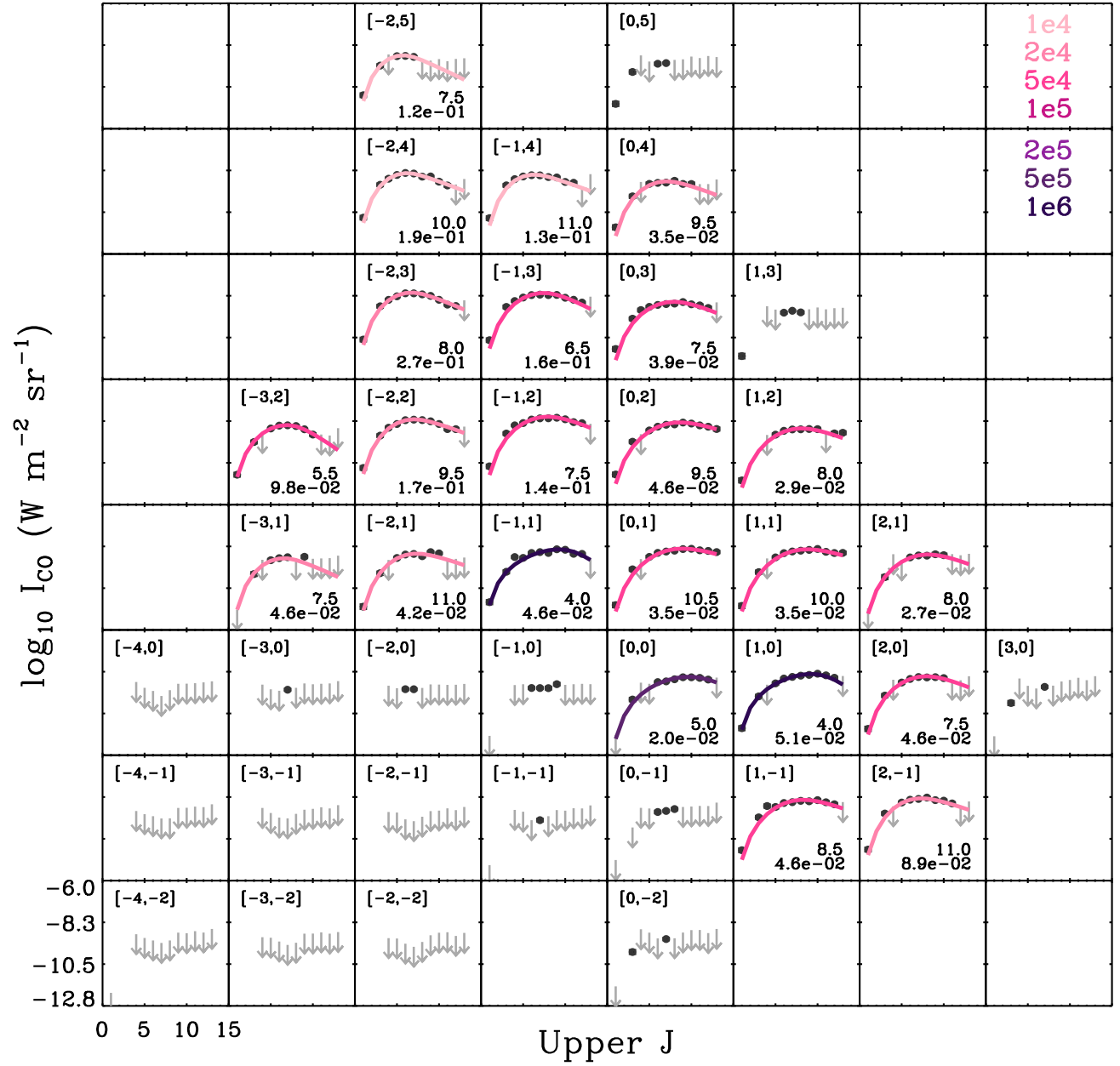

Fig. 14. Constrained shock models overlaid with the observed $\mathrm{CO}$ SLEDs. As in Fig. 5, the circles and downward arrows represent the measured intensities and $5 \sigma_{\mathrm{s}^{-}}$ based upper limits. A location with respect to the pixel that is closest to R136 is also indicated as a pair of numbers in the top left corner of each pixel (e.g., $[0,0]$ corresponds to $(\alpha, \delta)_{\mathrm{J} 2000}=\left(05^{\mathrm{h}} 38^{\mathrm{m}} 42^{\mathrm{s}}\right.$, $\left.-69^{\circ} 05^{\prime} 53^{\prime \prime}\right)$, and each pixel covers $\left.30^{\prime \prime}\right)$. The shock models with different $n_{\text {pre }}$ are presented in different colors (darker shades for higher $n_{\text {pre }}$ ), and the exact $n_{\text {pre }}$ values (in $\mathrm{cm}^{-3}$ ) are summarized in the top right corner of this figure. Finally, the constrained shock velocities (in $\mathrm{km} \mathrm{s}^{-1}$ ) and beam filling factors (unitless) are shown in the bottom right corner of each pixel.
Overall, we found that single shock models with $n_{\text {pre }} \sim 10^{4}$ $10^{6} \mathrm{~cm}^{-3}, v_{\mathrm{s}} \sim 5-10 \mathrm{~km} \mathrm{~s}^{-1}, \Omega \sim 0.01-0.1$, and no UV radiation field reproduce our $\mathrm{CO}$ and $\mathrm{H}_{2} 0-0 \mathrm{~S}(3)$ observations reasonably well. The constrained $v_{\mathrm{s}}$ and $\Omega$ values seem to be consistent with previous studies as well $\left(v_{\mathrm{s}}\right.$ : the $30^{\prime \prime}$-scale $\mathrm{CO}(7-6)$ spectrum obtained by Pineda et al. (2012) shows a line width of $\sim 10 \mathrm{~km} \mathrm{~s}^{-1} ; \Omega$ : the ALMA CO(2-1) observations by Indebetouw et al. (2013) suggest $\Omega \lesssim 0.1$ ), implying that our final shock models are reasonable. Considering the degeneracy that persists in our modeling, we do not discuss the shock parameters on a pixel-by-pixel basis (our solutions in Fig. 14 should be considered as approximative models) and instead focus on large-scale trends. For example, we examined the parameter space of "good" shock models that reproduce our $\mathrm{CO}$ and $\mathrm{H}_{2} \mathrm{O}-0 \mathrm{~S}(3)$ observations within a factor of two and found that the top pixels $[-2,5],[-2,4]$, and $[-1,4]$ indeed likely have a lower density of 


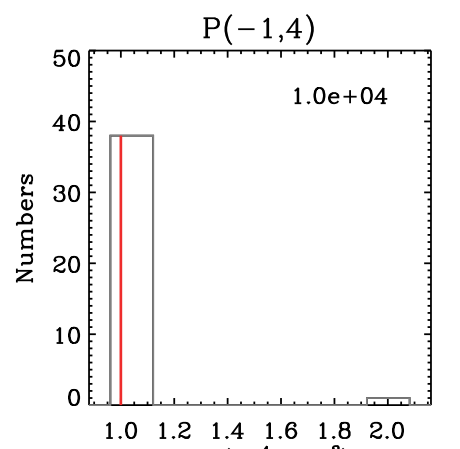

$\mathrm{n}_{\text {pre }}\left(10^{4} \mathrm{~cm}^{-3}\right)$
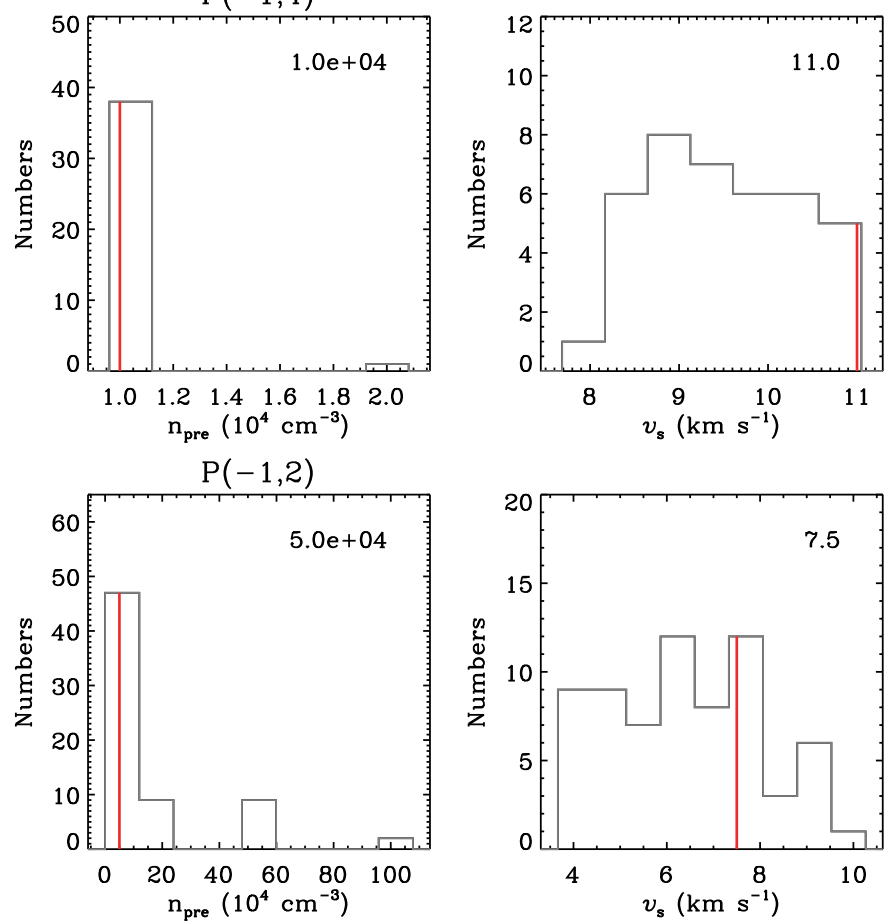

$\sim 10^{4} \mathrm{~cm}^{-3}$ compared to other pixels, based on a narrow distribution of $n_{\text {pre }}$ (Fig. 15).

While reproducing the observed CO (including $J=1-0$; the shocked medium produces $\sim 30-80 \%$ of the observed emission) and $\mathrm{H}_{2} \mathrm{O}-0 \mathrm{~S}(3)$ transitions reasonably well, the shock models predict quite faint fine-structure lines. Specifically, we found that the model-to-observed line ratios are only $\lesssim 4 \times 10^{-6}$, $\lesssim 0.01$, and $\lesssim 0.03$ for [C II] $158 \mu \mathrm{m}$, [C I] $370 \mu \mathrm{m}$, and [O I] $145 \mu \mathrm{m}$, respectively, implying that at least two ISM components are required to fully explain our observations of 30 Doradus. The likely possibility in the shock scenario for $\mathrm{CO}$ would be: (1) the low- $P$ PDR component $\left(10^{4}-10^{5} \mathrm{~K} \mathrm{~cm}^{-3}\right.$; Sect. 5.1.3) that primarily contributes to the observed [C II] $158 \mu \mathrm{m},[\mathrm{C}$ I] $370 \mu \mathrm{m},[\mathrm{O} \mathrm{I}] 145 \mu \mathrm{m}$, and FIR emission, and (2) the high- $P$ shock component $\left(10^{7}-10^{8} \mathrm{~K} \mathrm{~cm}^{-3}\right.$; Sect. 6.1 for details) that radiates intensely mainly in $\mathrm{CO}$. In the case of $\mathrm{H}_{2} 0-0 \mathrm{~S}(3)$, both components contribute, and the combined contributions are still consistent with the measured upper limits. The shock-toPDR ratio varies significantly from $\sim 0.1$ to $\sim 6$ for $\mathrm{H}_{2} 0-0 \mathrm{~S}(3)$, which could be partly due to the degeneracy we still have in $n_{\text {pre }}$ and $v_{\mathrm{s}}$.

In short, we conclude that low-velocity C-type shocks with $n_{\text {pre }} \sim 10^{4}-10^{6} \mathrm{~cm}^{-3}$ and $v_{\mathrm{s}} \sim 5-10 \mathrm{~km} \mathrm{~s}^{-1}$ could be another important source of excitation for $\mathrm{CO}$ in 30 Doradus. The shockcompressed $\left(P / k_{\mathrm{B}} \sim 10^{7}-10^{8} \mathrm{~K} \mathrm{~cm}^{-3}\right)$ CO-emitting clumps are likely $\sim 0.7-2 \mathrm{pc}$ in size and embedded within some low- $P$ $\left(P / k_{\mathrm{B}} \sim 10^{4}-10^{5} \mathrm{~K} \mathrm{~cm}^{-3}\right)$, UV-irradiated $\left(G_{\mathrm{UV}} \sim 10^{2}-10^{3}\right)$ ISM component that produces bright [C II] $158 \mu \mathrm{m}$, [C I] $370 \mu \mathrm{m}$, [O I] $145 \mu \mathrm{m}$, and FIR continuum emission. This low- $P$ PDR component fills a large fraction of our $30^{\prime \prime}$ pixels and provides up to $A_{\mathrm{V}} \sim 4-20 \mathrm{mag}$, shielding the shocked CO clumps from the dissociating UV radiation field. In the following sections, we present more detailed physical properties (e.g., density and temperature) of these shock and low- $P$ PDR components and compare them to those of the high- $P$ PDR component (Sect. 5.1.4), with an aim of probing the origin of $\mathrm{CO}$ emission in 30 Doradus.

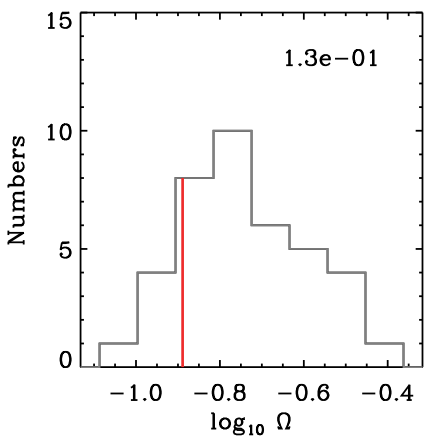

Fig. 15. Another illustration of the degeneracy in our shock modeling. The gray histograms show the selected shock models for two pixels $[-1,4]$ and $[-1,2]$ that reproduce our $\mathrm{CO}$ and $\mathrm{H}_{2} \quad 0-0 \quad \mathrm{~S}(3)$ observations within a factor of two (top and bottom; number of the selected models $=39$ and 67 , respectively). For each histogram, the final shock parameter we constrained in Sect. 5.3.3 is shown as the red solid line and summarized in the top right corner. A comparison between the top and bottom histograms clearly shows that $[-1,4]$ has a relatively narrow distribution of $n_{\text {pre }}$.

\section{Discussions}

\subsection{Physical conditions of the neutral gas}

\subsubsection{Low thermal pressure component}

We start our discussion by first presenting several physical quantities ( $n, T$, and line emissivities) of a representative low- $P$ PDR model $\left(A_{\mathrm{V}}=2 \mathrm{mag}, P / k_{\mathrm{B}}=10^{5} \mathrm{~K} \mathrm{~cm}^{-3}\right.$, and $\left.G_{\mathrm{UV}}=10^{3}\right)$ as a function of $A_{\mathrm{V}}$ in Fig. 16. As described throughout Sect. 5, this low- $P$ PDR component is primarily bright in [C II] $158 \mu \mathrm{m}$, [C I] $370 \mu \mathrm{m}$, [O I] $145 \mu \mathrm{m}$, and FIR continuum emission and is essential to fully reproduce our multi-wavelength data of 30 Doradus.

A close examination of the radial profiles in Fig. 16 suggests that $\mathrm{CO}$ emission mostly originates from a diffuse and relatively warm medium with $n \sim$ a few $100 \mathrm{~cm}^{-3}$ and $T \lesssim 100 \mathrm{~K}$. The CO abundance in this diffuse and extended (line-of-sight depth of $\sim 6$ pc) PDR component is low $\left(N(\mathrm{CO}) \sim\right.$ a few $\left.10^{13} \mathrm{~cm}^{-2}\right)$, which likely results from the following two aspects: (1) The slab of gas with relatively low dust extinction is illuminated by a strong UV radiation field. (2) The density is low. This low CO abundance is likely the primary reason for why the low- $P$ PDR component is so faint in $\mathrm{CO}$ emission.

\subsubsection{High thermal pressure component}

Our analysis above indicates that high densities and/or temperatures would be needed for the observed bright $\mathrm{CO}$ emission, and we found that it is indeed the case for the high- $P$ PDR and shock components. For an illustration, we then again select representative high- $P$ PDR $\left(A_{\mathrm{V}}=10 \mathrm{mag}, P / k_{\mathrm{B}}=6.7 \times 10^{8} \mathrm{~K} \mathrm{~cm}^{-3}\right.$, and $\left.G_{\mathrm{UV}}=10^{4}\right)$ and shock $\left(n_{\mathrm{pre}}=5 \times 10^{4} \mathrm{~cm}^{-3}, v_{\mathrm{s}}=8 \mathrm{~km} \mathrm{~s}^{-1}\right.$, and $G_{\mathrm{UV}}^{\prime}=0$ ) models and present their profiles in Fig. 16. We note that the shock profiles are different from those of the PDR models, in that they are shown as a function of the flow time through the shock structure (from the pre- to post-shock medium), rather than of dust extinction. 

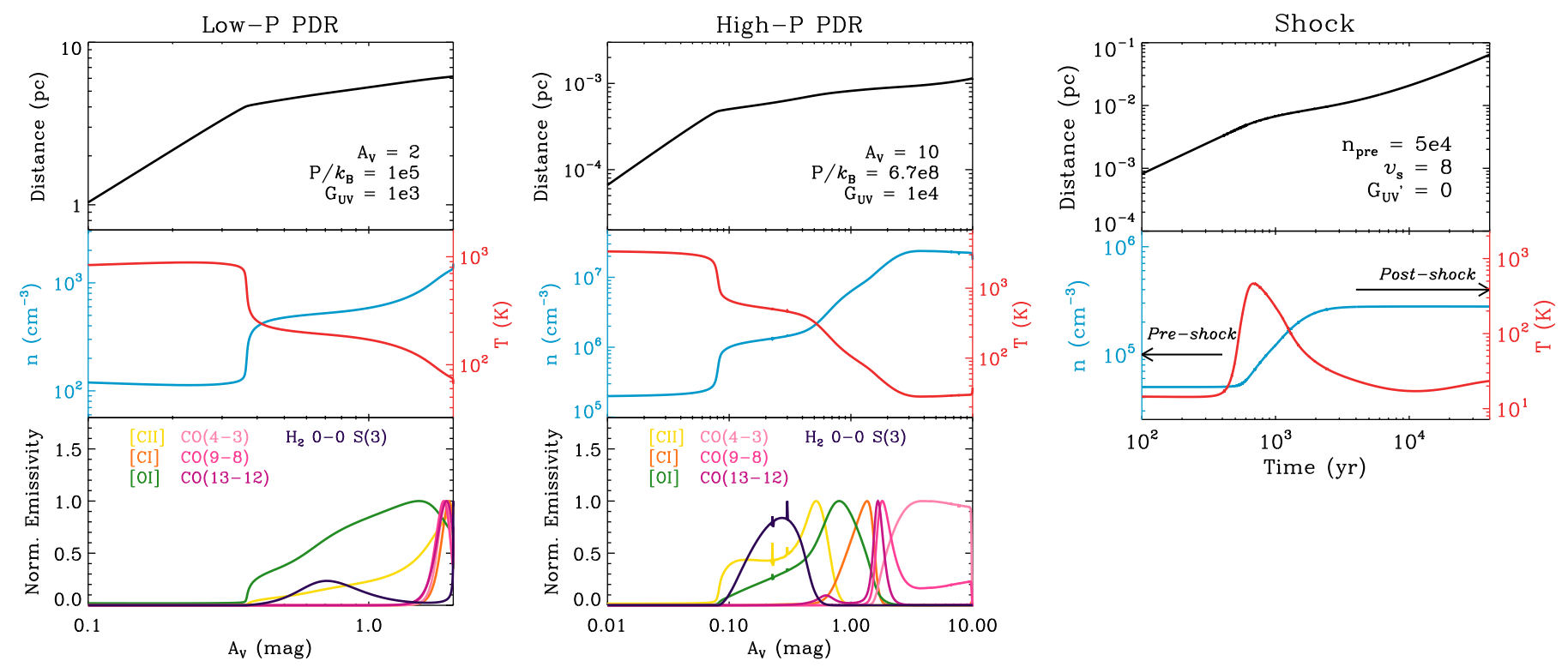

Fig. 16. Physical properties of the PDR and shock components in 30 Doradus. The following three models are presented as examples: (1) low- $P$ PDR with $A_{\mathrm{V}}=2 \mathrm{mag}, P / k_{\mathrm{B}}=10^{5} \mathrm{~K} \mathrm{~cm}^{-3}$, and $G_{\mathrm{UV}}=10^{3}$ (left), (2) high- $P$ PDR with $A_{\mathrm{V}}=10 \mathrm{mag}, P / k_{\mathrm{B}}=6.7 \times 10^{8} \mathrm{~K} \mathrm{~cm}{ }^{-3}$, and $G_{\mathrm{UV}}=10^{4}$ (middle), and (3) shock with $n_{\mathrm{pre}}=5 \times 10^{4} \mathrm{~cm}^{-3}, v_{\mathrm{s}}=8 \mathrm{~km} \mathrm{~s}^{-1}$, and $G_{\mathrm{UV}}^{\prime}=0$ (right). For the two PDR models, several physical quantities (top: distance; middle: density and temperature; bottom: normalized emissivities of [C II] $158 \mu \mathrm{m}$, [C I] $370 \mu \mathrm{m}$, and [O I] $145 \mu \mathrm{m}$, as well as selected CO and $\mathrm{H}_{2}$ transitions) are shown as a function of dust extinction. For the shock model, the distance (measured along the direction of propagation of the shock wave; top), as well as the density and temperature of the neutral fluid (bottom), are plotted as a function of flow time.

The profiles in Fig. 16 clearly show that the high- $P$ PDR has quite different conditions for $\mathrm{CO}$ emission compared to the low$P$ PDR. For example, we found that low- $J$ CO lines are emitted from a highly dense and cold medium with $n \gtrsim 10^{7} \mathrm{~cm}^{-3}$ and $T \sim$ $30 \mathrm{~K}$, while intermediate- and high- $J$ CO lines mainly arise from a relatively warm layer with $n \sim$ a few $10^{6} \mathrm{~cm}^{-3}$ and $T \sim 100 \mathrm{~K}$. The bright $\mathrm{CO}$ emission from this dense and highly compressed PDR component (line-of-sight depth of $\sim 10^{-3} \mathrm{pc}$ ) is likely due to abundant $\mathrm{CO}$ molecules $\left(N(\mathrm{CO}) \sim\right.$ a few $\left.10^{18} \mathrm{~cm}^{-2}\right)$, which result from the sufficient dust extinction $\left(A_{\mathrm{V}} \gtrsim 5 \mathrm{mag}\right)$ to protect $\mathrm{CO}$ from photodissociation, as well as from the high density.

The physical properties of the high- $P$ PDR component also appear to be slightly different from those of the low-velocity C-type shocks. Specifically, for the constrained shock models in Fig. 14, we found that the shock-compressed CO-emitting layer (line-of-sight depth of $\sim 10^{-2} \mathrm{pc}$ ) is less dense $\left(n \sim\right.$ a few $\left(10^{4}-\right.$ $\left.\left.10^{6}\right) \mathrm{cm}^{-3}\right)$ and less $\mathrm{CO}$ abundant $\left(N(\mathrm{CO}) \sim\right.$ a few $\left(10^{16}-10^{17}\right)$ $\left.\mathrm{cm}^{-2}\right)$, while having a higher temperature $(T \sim 100-500 \mathrm{~K})$ than the high- $P$ PDR counterpart.

\subsection{Source of the high thermal pressure}

Our analyses suggest that the observed CO emission in 30 Doradus most likely originates from strongly compressed regions, whose high pressure $\left(P / k_{\mathrm{B}} \sim 10^{7}-10^{9} \mathrm{~K} \mathrm{~cm}^{-3}\right)$ could be driven by either UV photons or shocks. Here we examine the likelihood of each case based on the known characteristics of 30 Doradus.

\subsubsection{Ultraviolet photons}

If UV photons are the dominant source of the high thermal pressure in the CO-emitting regions, we would expect a correlation between stellar properties (e.g., spectral type and stellar density) and the constrained PDR conditions. Such a correlation was indeed predicted recently by the photoevaporating PDR model of Bron et al. (2018), where one-dimensional hydrodynamics, UV radiative transfer, and time-dependent thermo-chemical evolution are calculated simultaneously for a molecular cloud exposed to an adjacent massive star. In this model, the UV-illuminated surface of the cloud can freely evaporate into the surrounding gas, and this photoevaporation at the ionization and dissociation fronts produces high pressure (up to $\sim 10^{9} \mathrm{~K} \mathrm{~cm}^{-3}$ ). One of the predicted aspects of the photoevaporating PDR was a linear relation between $P / k_{\mathrm{B}}$ and $G_{\mathrm{UV}}$, whose slope depends on the spectral type of the star (e.g., the $P / k_{\mathrm{B}}$-to- $G_{\mathrm{UV}}$ ratios of $\sim 5 \times 10^{3}$ and $\sim 8 \times 10^{4}$ for B- and O-type stars; higher ratios for hotter stars). This prediction seems to reproduce the observations of several Galactic PDRs (e.g., Joblin et al. 2018; Wu et al. 2018) and is shown in Fig. 17.

To evaluate whether UV photons are indeed responsible for the high thermal pressure in the CO-emitting regions, we examined the constrained high- $P$ PDR solutions in combination with the observed stellar properties. As an illustration, the minimum and maximum values of $P / k_{\mathrm{B}}$ and $G_{\mathrm{Uv}}$ are indicated in Fig. 17 as bars in different colors depending on star counts. Here the star counts were estimated by counting the number of stars that fall into each $30^{\prime \prime}$ FTS pixel $\left(\sim 1.3 \times 10^{4}\right.$ stars we used for the derivation of $G_{\text {stars }}$ were considered; Fig. 7 and Appendix B) and were found to vary by a factor of $\sim 40$, from 2 to 88 , for the eight pixels in our consideration. The measured $P / k_{\mathrm{B}}$ and $G_{\mathrm{UV}}$ values of 30 Doradus appear to be in reasonably good agreement with the predictions from Bron et al. (2018), but a close examination revealed that some of the observed trends are actually against expectations for UV-driven high pressure. For example, the pixels $[-2,2]$ and $[0,1]$ have the minimum and maximum star count, respectively, yet their thermal pressures are comparable $(\sim(0.5-$ 1) $\left.\times 10^{9} \mathrm{~K} \mathrm{~cm}^{-3}\right)$. Considering that the high- $P$ PDR components of both pixels are likely to be equally close to the plane of R136 (inferred from similar $G_{\mathrm{UV}}$ and $G_{\text {stars }}$ values; Fig. 12), it is indeed difficult to reconcile the comparable thermal pressures with the difference in star counts by a factor of $\sim 40$. In addition, $[0,1]$ 


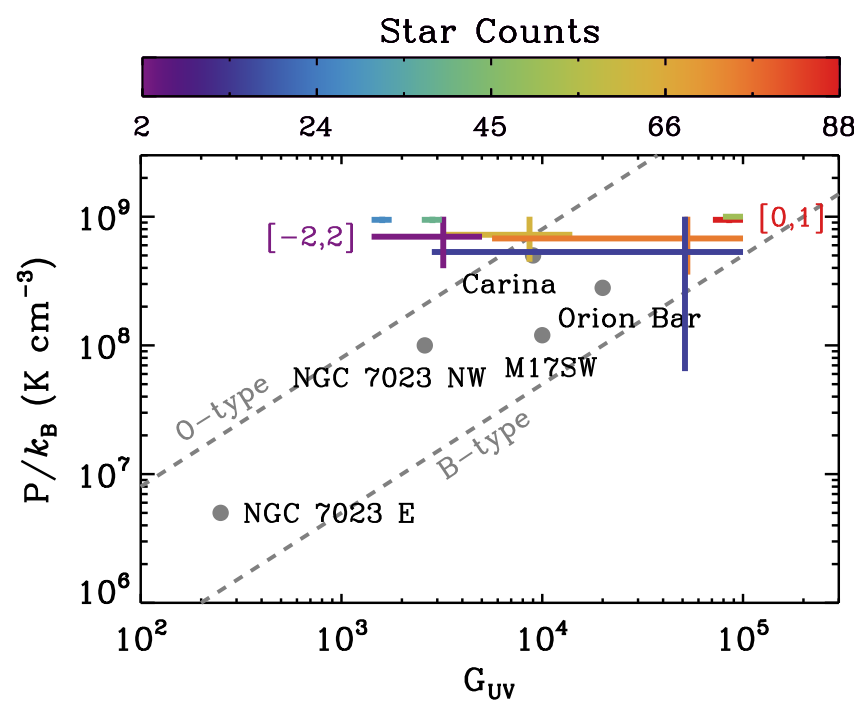

Fig. 17. $P / k_{\mathrm{B}}$ as a function of $G_{\mathrm{UV}}$ for various Galactic and extragalactic sources. The high- $P$ PDR conditions for $\mathrm{CO}$ emission in 30 Doradus are presented as the bars (same as in Fig 12) in different colors depending on star counts, while other sources are shown as the gray circles (Orion Bar and NGC 7023 NW from Joblin et al. 2018; Carina Nebula from Wu et al. 2018; NGC 7023 E from Köhler et al. 2014; M17SW from Pérez-Beaupuits et al. 2010). In addition, the two pixels we discuss in the main text, $[-2,2]$ and $[0,1]$, are indicated, along with the predictions from Bron et al. (2018) for B- and O-type stars (gray dashed lines; $P / k_{\mathrm{B}}-$ to- $G_{\mathrm{UV}}$ ratio $=5 \times 10^{3}$ and $8 \times 10^{4}$ respectively).

has a $P / k_{\mathrm{B}}$-to- $G_{\mathrm{UV}}$ ratio that is a factor of $\sim 20$ lower than that of $[-2,2]$, even though it has a greater number of hotter stars (13 stars with $T_{\text {eff }} \geq 4 \times 10^{4} \mathrm{~K}$ exist for [0,1], while [-2,2] has none). This result is in contrast with what is predicted by Bron et al. (2018).

In summary, we conclude that while the Meudon PDR model reproduces the observed $\mathrm{CO}$ lines, the constrained PDR conditions are not in line with what we would expect based on the stellar content of 30 Doradus. This conclusion however is tentative and requires further examination, since our current analyses have several limitations. For example, we analyzed the $\mathrm{CO}$ and fine-structure line observations of 30 Doradus using two PDR components. In reality, there would be a complicated mixture of multiple components on $\sim 10 \mathrm{pc}$ scales, and spatially- and spectrally resolved observations of $\mathrm{CO}$ and other neutral gas tracers (e.g., $\mathrm{HCO}^{+}$and $\mathrm{HCN}$ as dense gas tracers) would be needed to fully assess the impact of UV photons on CO in 30 Doradus. In addition, we compared the PDR properties of 30 Doradus with Bron et al. (2018), whose predictions are mainly for individual photoevaporating PDRs. To thoroughly examine whether UV photons are the dominant source of the high pressure for $\mathrm{CO}$ in 30 Doradus, a collective role of UV photons on larger scales must be considered, which would require simulations of multiple star clusters.

\subsubsection{Low-velocity shocks}

In the case where low-velocity shocks are the origin of the high thermal pressure in the CO-emitting regions, stellar winds from hot luminous stars could provide the required mechanical energy to drive the shocks. To examine such a possibility, we calculated the total energy dissipated by shocks $\left(E_{\mathrm{s}}\right)$ using our constrained models in Sect. 5.3.3 (Lee et al. 2016 for details on the calculation) and compared this with the stellar wind energy $\left(E_{\mathrm{w}}\right)$ of $\sim 500 \mathrm{~W}-\mathrm{R}$ and OB-type stars from Doran et al. (2013) (Fig. 18 left). For our comparison, the shock timescale of 0.1 Myr (typical time needed for the shocked medium to return to equilibrium) and the wind timescale of $2 \mathrm{Myr}$ (average OB stellar lifetime) were assumed, essentially following Doran et al. (2013) and Lee et al. (2016). When considering $\sim 150$ stars that fall into the 23 pixels where our shock solutions exist, we found that stellar winds from these stars can inject the total mechanical energy of $\sim 10^{52} \mathrm{erg}$, which would be sufficient to drive the low-velocity shocks dissipating $\sim 10^{50}$ erg in the region. These total energies of $\sim 10^{52}$ erg and $\sim 10^{50}$ erg were derived by simply summing up $E_{\mathrm{w}}$ and $E_{\mathrm{s}}$ over the 23 pixels under consideration. Interestingly, the left panel of Fig. 18 shows that the shock and wind energies have contrasting spatial distributions: $E_{\mathrm{s}}$ varies smoothly across the region, while $E_{\mathrm{W}}$ is highly concentrated around R136. To quantify this difference, we calculated $E_{\mathrm{w}}$ on a pixel-by-pixel basis by summing $E_{\mathrm{w}}$ values of all W-R and OB-type stars that fall into each FTS pixel and compared this to $E_{\mathrm{s}}$ (Fig. 18 right). As discussed immediately above, the right panel of Fig. 18 clearly demonstrates that $E_{\mathrm{s}}$ is relatively uniform with a variation of a factor of ten, while $E_{\mathrm{w}}$ changes by a factor of $\sim 2000$ ( $E_{\mathrm{w}}>10^{51} \mathrm{erg}$ coincides with R136 and its adjacent pixels). This highly concentrated distribution of $E_{\mathrm{w}}$ was also noted by Doran et al. (2013). Doran et al. (2013) found that $75 \%$ of the total wind luminosity is contained within $20 \mathrm{pc}$ of R136, suggesting that stellar winds are likely not the main driver of the low-velocity shocks.

In addition to stellar winds from hot luminous stars, $\mathrm{SNe}$ can inject a large amount of mechanical energy into the surrounding ISM $\left(\sim 10^{51}\right.$ erg per SNe; e.g., Hartmann 1999). So far 59 SNRs have been identified in the LMC (Maggi et al. 2016), and 30 Doradus harbors only one SNR, N157B at $(\alpha, \delta)_{\mathrm{J} 2000}=\left(05^{\mathrm{h}} 37^{\mathrm{m}} 47^{\mathrm{s}},-69^{\circ} 10^{\prime} 20^{\prime \prime}\right)($ Chen et al. 2006). Such a low number of SNRs is surprising, considering that 30 Doradus hosts $\sim 25 \%$ of the massive stars in the LMC (Kennicutt \& Hodge 1986). By assuming that core-collapsed SNRs closely follow active star formation (25 such SNRs in the LMC; Maggi et al. 2016), we can then estimate the expected number of $25 \times$ $0.25 \sim 6$ SNRs in 30 Doradus, which could have been missed due to their low surface brightness and/or the crowdedness in the 30 Doradus region. While our estimate is uncertain, it is indeed consistent with the roughly half-dozen high-velocity ionized bubbles in 30 Doradus (likely blown up by SNe; e.g., Chu \& Kennicutt 1994) and implies that SNe could provide sufficient energy to drive the low-velocity shocks. But again, as in the case of stellar winds, the relatively uniform distribution of $E_{\mathrm{s}}$ would be difficult to explain in the framework of SNe-driven shocks.

Our results so far suggest that the low-velocity shocks in 30 Doradus likely originate from nonstellar sources. This conclusion is also consistent with the fact that 30 Doradus and N159W have comparable $E_{\mathrm{s}}$ on $\sim 10 \mathrm{pc}$ scales (our FTS pixel size; Fig. 18 right) despite a large difference in the number of massive young stars: $~ 1100$ in 30 Doradus versus $\sim 150$ in N159W (Fariña et al. 2009; Doran et al. 2013). The comparable $E_{\mathrm{s}}$ values between 30 Doradus and N159W would in turn suggest that large-scale processes ( $\gtrsim 600 \mathrm{pc}$; distance between 30 Doradus and N159W) are likely the major source of the low-velocity shocks, and the kiloparsec-scale injection of significant energy into the Magellanic Clouds has indeed been suggested by previous power spectrum analyses (e.g., Elmegreen et al. 2001; Nestingen-Palm et al. 2017). One of the possible processes for energy injection on kiloparsec scales is the complicated interaction between the Milky Way and the Magellanic Clouds. While the dynamics of the entire Magellanic System (two Magellanic Clouds 

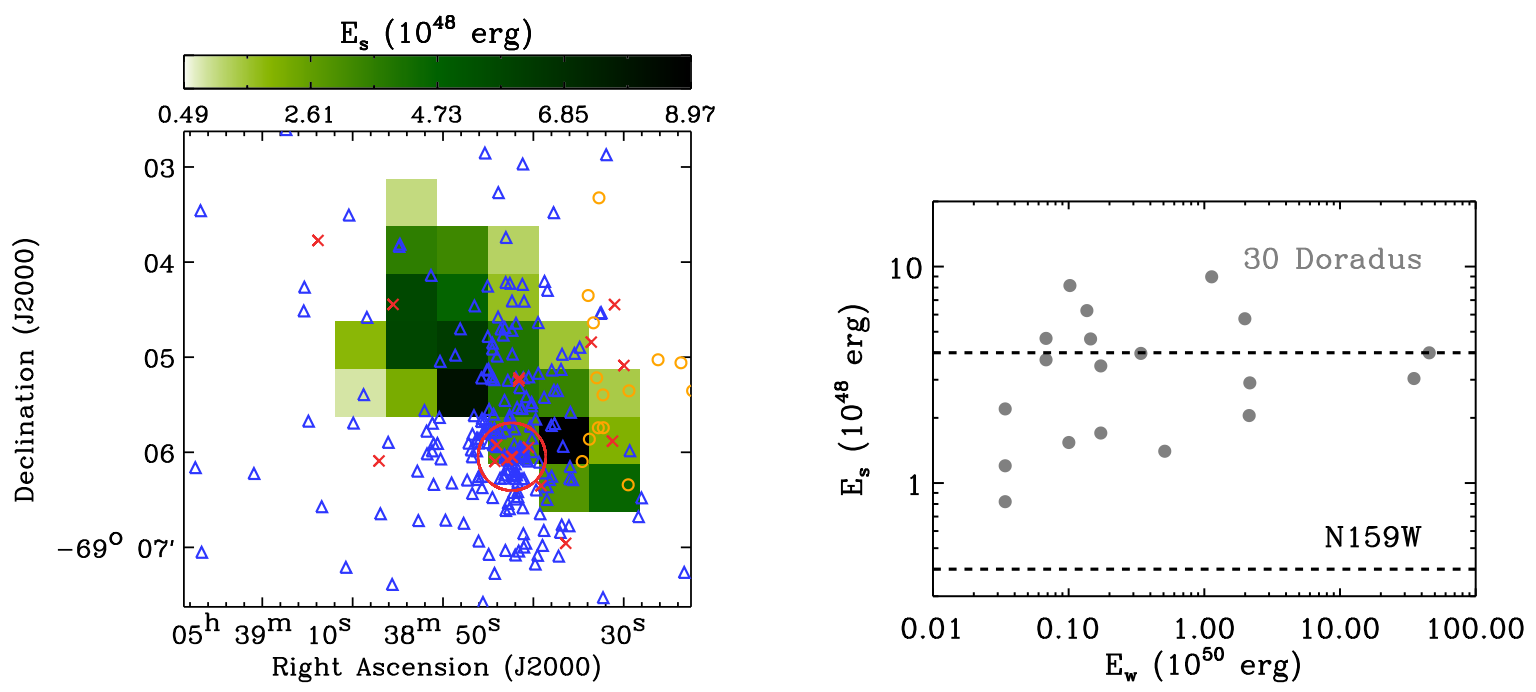

Fig. 18. Left: $E_{\mathrm{s}}$ of the final shock models (shown in Fig. 14) is presented along with $\sim 500$ stars for which $E_{\mathrm{w}}$ estimates are available $\left(E_{\mathrm{w}} \geq\right.$ $10^{50} \mathrm{erg}$ as the red crosses, $10^{49} \mathrm{erg} \leq E_{\mathrm{w}}<10^{50} \mathrm{erg}$ as the orange circles, and $E_{\mathrm{s}}<10^{49} \mathrm{erg}$ as the blue triangles; Doran et al. 2013). The location of R136 is also indicated by the red circle. Right: $E_{\mathrm{s}}$ as a function of $E_{\mathrm{w}}$. The data points for 30 Doradus are shown in gray, while the range of $E_{\mathrm{s}}$ estimated by Lee et al. (2016) for N159W is overlaid as the black dashed line.

and gaseous structures surrounding them, i.e., the Stream, the Bridge, and the Leading Arm) is still a subject of active research, it is well known that the southeastern $\mathrm{H}$ I overdensity region where 30 Doradus and N159W are located (Fig. 1) is strongly perturbed (e.g., Luks \& Rohlfs 1992) and likely influenced by tidal and/or ram-pressure stripping between the Milky Way and the Magellanic Clouds (e.g., D’Onghia \& Fox 2016). Such an energetic interplay between galaxies can deposit a large amount of mechanical energy, which would then cascade down to small scales and low velocities, as witnessed in both local and highredshift interacting systems (e.g., Appleton et al. 2017; Falgarone et al. 2017).

Finally, we note that low-velocity shocks would be pervasive in the LMC if they indeed arise from kiloparsec-scale processes. These shocks would have a negligible impact on the low- $P$ PDR component in 30 Doradus however, since the shocks would compress only a fraction of this diffuse and extended gas component (e.g., the line-of-sight depth of $\sim 10^{-2} \mathrm{pc}$ and $\sim 6 \mathrm{pc}$ for the high- $P$ shock and low-P PDR component, respectively; Sect. 6.1).

\subsection{CO spectral line energy distributions as a probe of the excitation mechanisms of warm molecular gas}

We analyzed the observed CO SLEDs of 30 Doradus with an aim of probing the excitation mechanisms of warm molecular gas in star-forming regions, and our results show that CO SLEDs alone cannot be used to differentiate between heating sources. For example, the observed CO SLEDs significantly change across 30 Doradus ( $J_{\mathrm{p}}=6-5$ to $10-9$ and $\alpha=0.4-1.8$; Sect. 4.2 ), and our PDR and shock modeling suggest that these varying CO SLEDs mostly reflect the changes in physical conditions (e.g., temperature and density), rather than underlying excitation mechanisms. The fact that N159W has systematically different CO SLEDs ( $J_{\mathrm{p}}=4-3$ to $7-6$ and $\alpha=0.3-0.7$; Lee et al. 2016) yet likely shares the same excitation mechanism as 30 Doradus also supports our conclusion. In addition to $\mathrm{CO}$ lines, complementary constraints (e.g., fine-structure lines, FIR luminosity, and properties of massive young stars) were then found to be highly essential to examine the excitation mechanisms in detail and evaluate their feasibility. All in all, our study demonstrates that one should take a comprehensive approach when interpreting multi-transition $\mathrm{CO}$ observations in the context of probing the excitation sources of warm molecular gas (e.g., Mashian et al. 2015; Indriolo et al. 2017; Kamenetzky et al. 2017).

Another key result from our work is the crucial role of shocks in $\mathrm{CO}$ heating. As described in Sect. 1, both Galactic and extragalactic studies have highlighted the importance of mechanical heating for CO, and our N159W and 30 Doradus analyses show that mechanical heating by low-velocity shocks $\left(\sim 10 \mathrm{~km} \mathrm{~s}^{-1}\right)$ is indeed a major contributor to the excitation of molecular gas on $\sim 10 \mathrm{pc}$ scales. What remains relatively uncertain is the source of shocks. While we concluded that low-velocity shocks in N159W and 30 Doradus likely originate from large-scale processes such as the complex interaction between the Milky Way and the Magellanic Clouds, this hypothesis should be verified by observing independent shock tracers throughout the LMC, such as $\mathrm{SiO}$ and $\mathrm{H}_{2}$ transitions. Such observations would be possible with current and upcoming facilities (e.g., ALMA, SOFIA, and JWST), providing insight into the injection and dissipation of mechanical energy in the ISM. These observations will also further test our tentative rejection of UV photons as the main heating source for $\mathrm{CO}$ (Sect. 6.2.1).

\section{Summary}

In this paper, we present Herschel SPIRE FTS observations of 30 Doradus, the most extreme starburst region in the Local Universe with more than 1000 massive young stars. To examine the physical conditions and excitation mechanisms of molecular gas, we combined the FTS CO observations (CO $J=4-3$ to $J=13-$ 12) with other tracers of gas and dust and analyzed them on $42^{\prime \prime}$ or $\sim 10$ pc scales using the state-of-the-art Meudon PDR and Paris-Durham shock models. Our main results are as follows.

1. In our FTS observations, important cooling lines in the ISM, such as CO rotational transitions (from $J=4-3$ to $J=13-12$ ), [C I] $370 \mu \mathrm{m}$, and [N II] $205 \mu \mathrm{m}$, were clearly detected.

2. We constructed CO SLEDs on a pixel-by-pixel basis by combining the FTS observations with ground-based $\mathrm{CO}(1-$ 0 ) and $\mathrm{CO}(3-2)$ data and found that the CO SLEDs vary 
considerably across 30 Doradus. These variations include the changes in the peak transition $J_{\mathrm{p}}$ (from $J=6-5$ to $J=10-9)$, as well as in the slope characterized by the high-to-intermediate $J$ ratio $\alpha$ (from $\sim 0.4$ to $\sim 1.8$ ).

3. To evaluate the impact of $\mathrm{UV}$ photons on $\mathrm{CO}$, we performed Meudon PDR modeling and showed that $\mathrm{CO}$ emission in 30 Doradus could arise from $\sim 0.7-2$ pc scale PDR clumps with $A_{\mathrm{V}} \gtrsim 5 \mathrm{mag}, P / k_{\mathrm{B}} \sim 10^{8}-10^{9} \mathrm{~K} \mathrm{~cm}^{-3}$, and $G_{\mathrm{UV}} \sim 10^{3}-$ $10^{5}$. Interestingly, these PDR clumps are quite faint in [C II] $158 \mu \mathrm{m}$ and FIR dust continuum emission, and we found that another PDR component with lower $A_{\mathrm{V}} \sim 2 \mathrm{mag}, P / k_{\mathrm{B}} \sim \mathrm{a}$ few $\left(10^{4}-10^{5}\right) \mathrm{K} \mathrm{cm}^{-3}$, and $G_{\mathrm{UV}} \sim$ a few $\left(10^{2}-10^{3}\right)$ (filling a large fraction of $\sim 10 \mathrm{pc}$ FTS pixels) is required to explain the observed fine-structure lines ([C II] $158 \mu \mathrm{m}$, [C I] $370 \mu \mathrm{m}$, and [O I] $145 \mu \mathrm{m}$ ) and FIR luminosity. The constrained properties of the high- $P$ PDR clumps however are not consistent with what we would expect based on the stellar content of 30 Doradus, and we therefore tentatively concluded that UV photons are likely not the primary heating source for CO.

4. Based on the observed X-ray and $\gamma$-ray properties of 30 Doradus, we concluded that X-rays and cosmic-rays likely play a minor role in $\mathrm{CO}$ heating.

5. Our Paris-Durham shock modeling showed that the observed CO SLEDs of 30 Doradus can be reproduced by low-velocity C-type shocks with $n_{\text {pre }} \sim 10^{4}-10^{6} \mathrm{~cm}^{-3}$ and $v_{\mathrm{s}} \sim 5-10 \mathrm{~km} \mathrm{~s}^{-1}$. The shock-compressed $\left(P / k_{\mathrm{B}} \sim\right.$ $10^{7}-10^{8} \mathrm{~K} \mathrm{~cm}^{-3}$ ) CO-emitting clumps on $\sim 0.7-2 \mathrm{pc}$ scales are likely well-shielded from dissociating UV photons and embedded within the low- $P$ PDR component that emits brightly in [C II] $158 \mu \mathrm{m}$, [C I] $370 \mu \mathrm{m}$, [O I] $145 \mu \mathrm{m}$, and FIR continuum emission. Considering the properties of massive young stars in 30 Doradus, we excluded the stellar origin of low-velocity shocks and concluded that the shocks are likely driven by large-scale processes such as the interaction between the Milky Way and the Magellanic Clouds.

6. Our analyses suggest that the significant variations in the observed CO SLEDs of 30 Doradus mostly reflect the changes in physical conditions (e.g., temperature and density) rather than underlying excitation mechanisms. This implies that the shape of CO SLEDs alone cannot be used as a probe of heating sources.

While large-scale low-velocity shocks were suggested as the dominant heating source of $\mathrm{CO}$ in 30 Doradus, we note that our conclusion was based on analyses at a scale of $\sim 10 \mathrm{pc}$. As Indriolo et al. (2017) pointed out, CO SLEDs strongly depend on spatial scales, and the way in which the spatial scale of CO observations affects the interpretation of heating sources is currently unclear. To obtain a more comprehensive picture of the nature of warm molecular gas in star-forming regions, it is hence critical to analyze high spatial and spectral resolution $\mathrm{CO}$ observations over a large area in combination with complementary constraints such as PDR and shock tracers as well as stellar properties.

Acknowledgements. We would like to thank the anonymous referee for constructive comments which improved this work. We also thank E. Bron, B. Godard, C. Matzner, E. Roueff, A. Tielens, and S. Viti for helpful discussions and acknowledge support from the SYMPATICO grant of the French Agence Nationale de la Recherche (ANR-11-BS56-0023), the PCMI program of the French Centre National de la Recherche Scientifique, the sub-project A6 of the Collaborative Research Council 956 funded by the Deutsche Forschungsgemeinschaft, and the Emmy Noether grant KR4801/1-1 funded by the Deutsche Forschungsgemeinschaft. PACS has been developed by a consortium of institutes led by MPE (Germany) and including UVIE (Austria); KU Leuven, CSL, IMEC (Belgium); CEA, LAM (France); MPIA (Germany); INAF-IFSI/OAA/OAP/OAT, LENS, SISSA (Italy); IAC (Spain). This development has been supported by the funding agencies BMVIT (Austria),
ESA-PRODEX (Belgium), CEA/CNES (France), DLR (Germany), ASI/INAF (Italy), and CICYT/MCYT (Spain). SPIRE has been developed by a consortium of institutes led by Cardiff University (UK) and including University of Lethbridge (Canada); NAOC (China); CEA, LAM (France); IFSI, University of Padua (Italy); IAC (Spain); Stockholm Observatory (Sweden); Imperial College London, RAL, UCL-MSSL, UKATC, University of Sussex (UK); and Caltech, JPL, NHSC, University of Colorado (USA). This development has been supported by national funding agencies: CSA (Canada); NAOC (PR China); CEA, CNES, CNRS (France); ASI (Italy); MCINN (Spain); SNSB (Sweden); STFC, UKSA (UK); and NASA (USA).

\section{References}

Abdo, A. A., Ackermann, M., Ajello, M., et al. 2009, ApJ, 703, 1249 Abdo, A. A., Ackermann, M., Ajello, M., et al. 2010, A\&A, 512, A7 Appleton, P. N., Guillard, P., Togi, A., et al. 2017, ApJ, 836, 76 Bakes, E. L. O., \& Tielens, A. G. G. M. 1994, ApJ, 427, 822

Bron, E., Le Bourlot, J., \& Le Petit, F. 2014, A\&A, 569, A100 Bron, E., Le Petit, F., \& Le Bourlot, J. 2016, A\&A, 588, A27

Bron, E., Agúndez, M., Goicoechea, J. R., \& Cernicharo, J. 2018, A\&A, submitted [arXiv:1801.01547]

Burton, M. G., Hollenbach, D. J., \& Tielens, A. G. G. M. 1990, ApJ, 365, 620

Chen, Y., Wang, Q. D., Gotthelf, E. V., et al. 2006, ApJ, 651, 237

Chevance, M., Madden, S. C., Lebouteiller, V., et al. 2016, A\&A, 590, A36 Chu, Y.-H., \& Kennicutt, Jr. R. C. 1994, ApJ, 425, 720

Cormier, D., Madden, S. C., Lebouteiller, V., et al. 2015, A\&A, 578, A53

Crowther, P. A., \& Dessart, L. 1998, MNRAS, 296, 622

Crowther, P. A., Schnurr, O., Hirschi, R., et al. 2010, MNRAS, 408, 731

de Graauw, T., Helmich, F. P., Phillips, T. G., et al. 2010, A\&A, 518, L6

D’Onghia, E., \& Fox, A. J. 2016, ARA\&A, 54, 363

Doran, E. I., Crowther, P. A., de Koter, A., et al. 2013, A\&A, 558, A134

Draine, B. T. 1978, ApJS, 36, 595

Draine, B. T., \& McKee, C. F. 1993, ARA\&A, 31, 373

Elmegreen, B. G., Kim, S., \& Staveley-Smith, L. 2001, ApJ, 548, 749

Evans, C. J., Taylor, W. D., Hénault-Brunet, V., et al. 2011, A\&A, 530, A108

Falgarone, E., Zwaan, M. A., Godard, B., et al. 2017, Nature, 548, 430

Fariña, C., Bosch, G. L., Morrell, N. I., Barbá, R. H., \& Walborn, N. R. 2009, AJ, 138, 510

Flower, D. R., \& Pineau des Forêts, G. 2015, A\&A, 578, A63

Fulton, T. R., Baluteau, J.-P., Bendo, G., et al. 2010, SPIE Conf. Ser., 7731, 773134

Galliano, F. 2018, MNRAS, 476, 1445

Goicoechea, J. R., Pety, J., Cuadrado, S., et al. 2016, Nature, 537, 207

Goicoechea, J. R., Cuadrado, S., Pety, J., et al. 2017, A\&A, 601, L9

Griffin, M. J., Abergel, A., Abreu, A., et al. 2010, A\&A, 518, L3

Gusdorf, A., Anderl, S., Güsten, R., et al. 2012, A\&A, 542, L19

Gusdorf, A., Riquelme, D., Anderl, S., et al. 2015, A\&A, 575, A98

Habart, E., Dartois, E., Abergel, A., et al. 2010, A\&A, 518, L116

Hailey-Dunsheath, S., Sturm, E., Fischer, J., et al. 2012, ApJ, 755, 57

Hartmann, D. H. 1999, Proc. Natl. Acad. Sci., 96, 4752

Higdon, S. J. U., Devost, D., Higdon, J. L., et al. 2004, PASP, 116, 975

Hollenbach, D. J., Chernoff, D. F., \& McKee, C. F. 1989, in Infrared Spectroscopy in Astronomy, ed. E. Böhm-Vitense, ESA SP, 290

Indebetouw, R., de Messières, G. E., Madden, S., et al. 2009, ApJ, 694, 84

Indebetouw, R., Brogan, C., Chen, C.-H. R., et al. 2013, ApJ, 774, 73

Indriolo, N., \& McCall, B. J. 2012, ApJ, 745, 91

Indriolo, N., Neufeld, D. A., Gerin, M., et al. 2015, ApJ, 800, 40

Indriolo, N., Bergin, E. A., Goicoechea, J. R., et al. 2017, ApJ, 836, 117

Israel, F. P., Güsten, R., Meijerink, R., et al. 2014, A\&A, 562, A96

Joblin, C., Bron, E., Pinto, C., et al. 2018, A\&A, 615, A129

Johansson, L. E. B., Greve, A., Booth, R. S., et al. 1998, A\&A, 331, 857

Kamenetzky, J., Glenn, J., Rangwala, N., et al. 2012, ApJ, 753, 70

Kamenetzky, J., Rangwala, N., \& Glenn, J. 2017, MNRAS, 471, 2917

Kennicutt, R. C., \& Evans, N. J. 2012, ARA\&A, 50, 531

Kennicutt, Jr. R. C., \& Hodge, P. W. 1986, ApJ, 306, 130

Kim, S., Staveley-Smith, L., Dopita, M. A., et al. 1998, ApJ, 503, 674

Köhler, M., Habart, E., Arab, H., et al. 2014, A\&A, 569, A109

Larson, R. L., Evans, II, N. J., Green, J. D., \& Yang, Y.-L. 2015, ApJ, 806, 70

Le Bourlot J., Pineau des Forêts, G., \& Flower, D. R. 1999, MNRAS, 305, 802

Le Bourlot, J., Le Petit, F., Pinto, C., Roueff, E., \& Roy, F. 2012, A\&A, 541, A76

Lebouteiller, V., Bernard-Salas, J., Sloan, G. C., \& Barry, D. J. 2010, PASP, 122, 231

Lebouteiller, V., Cormier, D., Madden, S. C., et al. 2012, A\&A, 548, A91

Lee, M.-Y., Madden, S. C., Lebouteiller, V., et al. 2016, A\&A, 596, A85

Le Petit, F., Nehmé, C., Le Bourlot, J., \& Roueff, E. 2006, ApJS, 164, 506 
Lesaffre, P., Pineau des Forêts, G., Godard, B., et al. 2013, A\&A, 550, A106

Luks, T., \& Rohlfs, K. 1992, A\&A, 263, 41

Maggi, P., Haberl, F., Kavanagh, P. J., et al. 2016, A\&A, 585, A162

Makiwa, G., Naylor, D. A., Ferlet, M., et al. 2013, Appl. Opt., 52, 3864

Mashian, N., Sturm, E., Sternberg, A., et al. 2015, ApJ, 802, 81

Mathis, J. S., Mezger, P. G., \& Panagia, N. 1983, A\&A, 128, 212

Meijerink, R., Kristensen, L. E., Weiß, A., et al. 2013, ApJ, 762, L16

Meixner, M., Gordon, K. D., Indebetouw, R., et al. 2006, AJ, 132, 2268

Minamidani, T., Mizuno, N., Mizuno, Y., et al. 2008, ApJS, 175, 485

Nestingen-Palm, D., Stanimirović, S., González-Casanova, D. F., et al. 2017, ApJ, 845,53

Newville, M., Stensitzki, T., Allen, D. B., \& Ingargiola, A. 2014, LMFIT: NonLinear Least-Square Minimization and Curve-Fitting for Python Okada, Y., Güsten, R., Requena-Torres, M. A., et al. 2019, A\&A, 621, A62 Ott, S. 2010, ASP Conf. Ser., 434, 139

Papadopoulos, P. P., Zhang, Z.-Y., Xilouris, E. M., et al. 2014, ApJ, 788, 153

Pellegrini, E. W., Baldwin, J. A., \& Ferland, G. J. 2010, ApJS, 191, 160

Pellegrini, E. W., Smith, J. D., Wolfire, M. G., et al. 2013, ApJ, 779, L19

Pérez-Beaupuits, J. P., Spaans, M., Hogerheijde, M. R., et al. 2010, A\&A, 510, A87

Pilbratt, G. L., Riedinger, J. R., Passvogel, T., et al. 2010, A\&A, 518, L1

Pineda, J. L., Mizuno, N., Röllig, M., et al. 2012, A\&A, 544, A84

Poglitsch, A., Waelkens, C., Geis, N., et al. 2010, A\&A, 518, L2

Pon, A., Kaufman, M. J., Johnstone, D., et al. 2016, ApJ, 827, 107

Rangwala, N., Maloney, P. R., Glenn, J., et al. 2011, ApJ, 743, 94

Röllig, M., Ossenkopf, V., Jeyakumar, S., Stutzki, J., \& Sternberg, A. 2006 A\&A, 451, 917
Rosenberg, M. J. F., Kazandjian, M. V., van der Werf, P. P., et al. 2014, A\&A, 564, A126

Rosenberg, M. J. F., van der Werf, P. P., Aalto, S., et al. 2015, ApJ, 801, 72

Russell, S. C., \& Dopita, M. A. 1992, ApJ, 384, 508

Schaefer, B. E. 2008, AJ, 135, 112

Schirm, M. R. P., Wilson, C. D., Parkin, T. J., et al. 2014, ApJ, 781, 101

Selman, F. J., \& Melnick, J. 2013, A\&A, 552, A94

Selman, F., Melnick, J., Bosch, G., \& Terlevich, R. 1999, A\&A, 341, 98

Smith, J. D. T., Armus, L., Dale, D. A., et al. 2007, PASP, 119, 1133

Sternberg, A., \& Dalgarno, A. 1989, ApJ, 338, 197

Stock, D. J., Wolfire, M. G., Peeters, E., et al. 2015, A\&A, 579, A67

Stoerzer, H., Stutzki, J., \& Sternberg, A. 1996, A\&A, 310, 592

Swinyard, B. M., Polehampton, E. T., Hopwood, R., et al. 2014, MNRAS, 440, 3658

Tielens, A. G. G. M., \& Hollenbach, D. 1985, ApJ, 291, 722

Townsley, L. K., Broos, P. S., Feigelson, E. D., et al. 2006a, AJ, 131, 2140

Townsley, L. K., Broos, P. S., Feigelson, E. D., Garmire, G. P., \& Getman, K. V. 2006b, AJ, 131, 2164

van der Tak, F. F. S., Black, J. H., Schöier, F. L., Jansen, D. J., \& van Dishoeck, E. F. 2007, A\&A, 468, 627

van der Werf, P. P., Isaak, K. G., Meijerink, R., et al. 2010, A\&A, 518, L42

Weingartner, J. C., \& Draine, B. T. 2001, ApJS, 134, 263

Weingartner, J. C., Draine, B. T., \& Barr, D. K. 2006, ApJ, 645, 1188

Wong, T., Hughes, A., Ott, J., et al. 2011, ApJS, 197, 16

Wu, R., Madden, S. C., Galliano, F., et al. 2015, A\&A, 575, A88

Wu, R., Bron, E., Onaka, T., et al. 2018, A\&A, 618, A53 


\section{Appendix A: FTS data}

In Fig. A.1, we present the FTS CO, [C I], and [N II] integrated intensity images of 30 Doradus. All images are at resolution of $42^{\prime \prime}\left(\sim 10 \mathrm{pc}\right.$ at the LMC distance) with a pixel size of $30^{\prime \prime}$. In each image, the spectra of individual pixels are overlaid in red (detections with $S / N_{\mathrm{s}}>5$ ) and blue (nondetections with
$\left.S / N_{\mathrm{s}} \leq 5\right)$. These spectra are plotted with the same $x$ - and $y$-axis ranges (in $\mathrm{GHz}$ and $10^{-18} \mathrm{~W} \mathrm{~m}^{-2} \mathrm{~Hz}^{-1} \mathrm{sr}^{-1}$ ), which can be found in the top left corner of the image with an example spectrum. The example spectrum is from the pixel that was observed with the central SLWC3 and SSWD4 detectors of the first jiggle position of the Obs. ID $=1342219550$ (yellow and orange crosses).
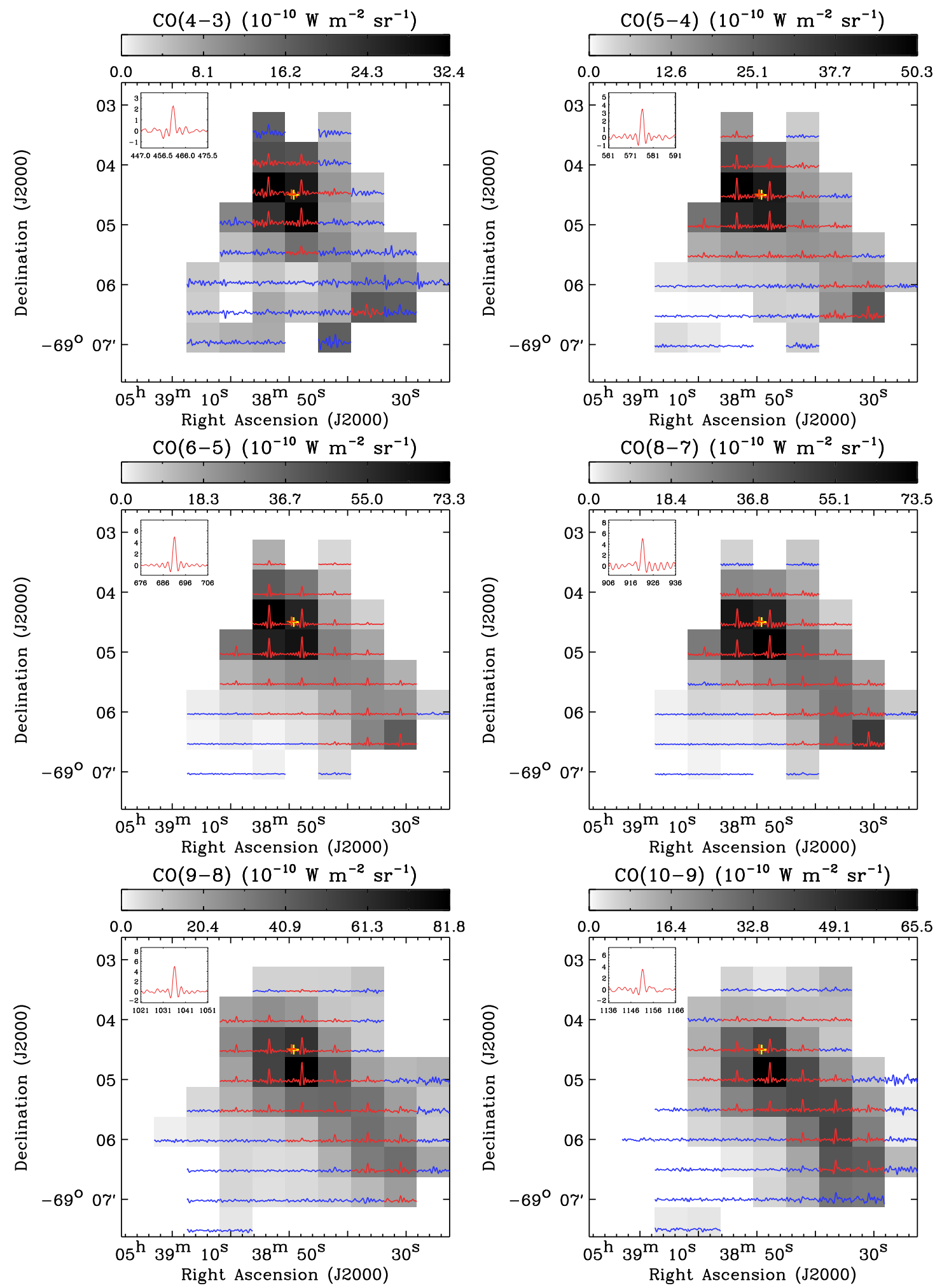

Fig. A.1. FTS CO, [C I] and [N II] integrated intensity images of 30 Doradus. See Appendix A for details on these figures. 
M.-Y. Lee et al.: Radiative and mechanical feedback into the molecular gas in the Large Magellanic Cloud. II. 30 Doradus
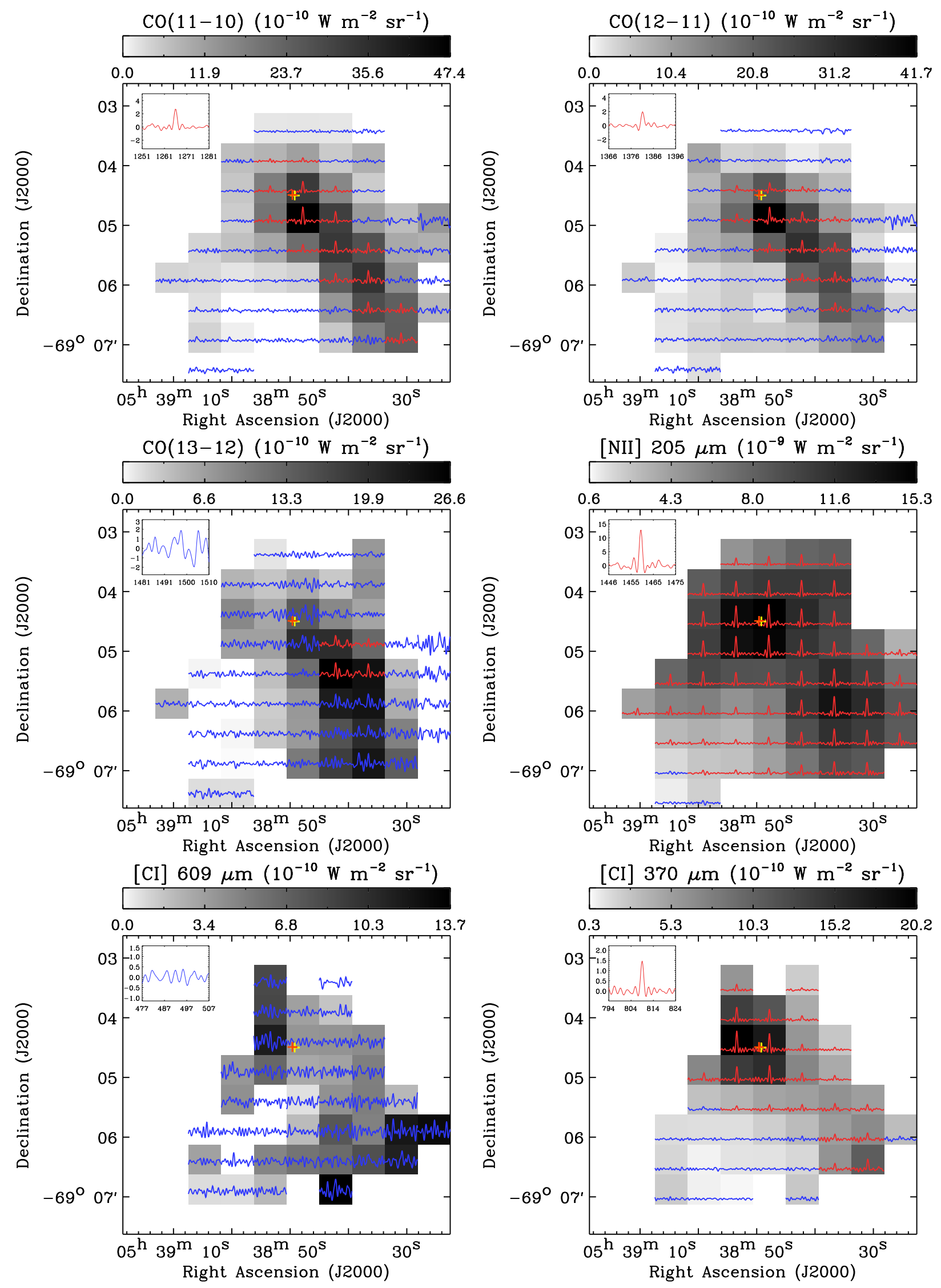

Fig. A.1. continued. 


\section{Appendix B: Stellar UV radiation field}

To derive the stellar UV radiation field $G_{\text {stars }}$ of 30 Doradus, we essentially followed Chevance et al. (2016) and provide a summary of our derivation here. First, we cross-matched the catalogs of massive stars (W-R and OB-type stars) published by Crowther \& Dessart (1998), Selman et al. (1999), and Doran et al. (2013) and extracted the temperatures of $\sim 1.3 \times 10^{4}$ sources. The cata$\log$ by Doran et al. (2013) was used as our main reference when possible, since it likely provides more reliable estimates of stellar properties based on spectroscopic observations, and the other two catalogs were used to complement it. We then integrated a blackbody from 912 to $2400 \AA$ for each star to be consistent with the definition of $G_{\mathrm{UV}}$ in the Meudon PDR model and calculated $G_{\text {stars }}$ for each $30^{\prime \prime}$ pixel of a $5^{\prime} \times 5^{\prime}$ image (matching the coverage and pixel size of the FTS maps) by assuming that all the stars lie on the same plane as R136 and summing the UV fluxes of the stars. The derived $G_{\text {stars }}$ on the plane of R136 is presented in Fig. 7 and can be considered as the maximum incident UV radiation field we expect, since no absorption was taken into account. While absorption by dust would have an important impact on the derivation of $G_{\text {stars }}$, estimating absorption is currently not straightforward due to the lack of information on the location of absorbers.

Compared to Chevance et al. (2016), the only difference is our usage of the catalog by Doran et al. (2013). This results in $\sim 50 \%$ stronger UV radiation field on average, suggesting that a factor of two or so uncertainty could arise from the selection of stellar catalogs. Another source of uncertainty is our assumption of the stellar distribution, and we examined this issue by deriving $G_{\text {stars }}$ with a random three-dimensional distribution of the stars. We then found that the assumption of the stars on the same plane tends to underestimate $G_{\text {stars }}$ by up to $\sim 30 \%$.

\section{Appendix C: PDR contribution to FIR emission}

Far-infrared emission can originate not only from PDRs (neutral gas), but also from H II regions (ionized gas). To be properly compared to the predictions from the Meudon PDR model, our $L_{\text {FIR }}$ derived from dust SED modeling (Sect. 3.5) then needs to be corrected for the contribution from the ionized medium. To do so, we used the PAH and [O III] $88 \mu \mathrm{m}$ images from Chevance et al. (2016) and performed the following steps (essentially what Chevance et al. 2016 did, but on $42^{\prime \prime}$ scales). First, we assumed that PAH and [O III] $88 \mu \mathrm{m}$ emission trace PDRs and $\mathrm{H}$ II regions respectively and adopted a linear relation $L_{\mathrm{FIR}}=\alpha L_{\mathrm{PAH}}+\beta L_{[\mathrm{O}}$ III] . The coefficients $(\alpha, \beta)=(4.6,8.8)$ were then derived by fitting a multiple regression model to all available pixels, and the PDRonly component of $L_{\mathrm{FIR}}$ was estimated as $L_{\mathrm{FIR}}^{\mathrm{PDR}}=L_{\mathrm{FIR}}-\beta L_{[\mathrm{O} \text { III] }}$. We note that our analysis is based on the assumption that the
PAH-to-dust mass ratio does not change in the PDRs and drops to zero in the $\mathrm{H}$ II regions.

This $L_{\mathrm{FIR}}^{\mathrm{PDR}}$ is what we used as an input for PDR modeling in Sect. 5.1, and we assigned $30 \%$ of $L_{\mathrm{FIR}}^{\mathrm{PDR}}$ as $1 \sigma$ uncertainties, considering the simple empirical relation we adopted. The resulting $L_{\mathrm{FIR}}^{\mathrm{PDR}}$ shows a good agreement with Chevance et al. (2016): we found that the PDR contribution to $L_{\mathrm{FIR}}$ ranges from $\sim 40$ to $\sim 80 \%$ across 30 Doradus, while Chevance et al. (2016) estimated $\sim 30$ to $\sim 90 \%$.

\section{Appendix D: Comparison with Chevance et al. (2016)}

As described in Sect. 2.2, Chevance et al. (2016) recently studied the properties of PDRs in 30 Doradus on $12^{\prime \prime}$ scales by performing Meudon PDR modeling of [C II] $158 \mu \mathrm{m}$, [O I] $145 \mu \mathrm{m}$, and FIR emission. Since we employed essentially the same datasets and modeling approach for our analyses in Sect. 5.1.3, it is important to double-check that our results are consistent with those in Chevance et al. (2016).

As for the spatial distributions of the PDR parameters (e.g., peak locations of $P, G_{\mathrm{UV}}$, and $\Omega$ ), we found that our results are consistent with Chevance et al. (2016). However, a comparison of the absolute values showed a large discrepancy, as can be seen in Fig. D.1. Specifically, we noticed that our $P$ and $G_{U V}$ distributions primarily trace the lower part of the Chevance et al. (2016) histograms, while the opposite is the case for $\Omega$.

This large discrepancy in the absolute values of the PDR parameters could result from several differences between the two studies, such as resolution (42" versus $12^{\prime \prime}$ ), spatial coverage (our maps are smaller), and slightly inconsistent PDR models (version 1.5.2 with $A_{\mathrm{V}} \sim 2$ mag versus version 1.6.0 with $A_{\mathrm{V}}=3 \mathrm{mag}$ ). Among these possibilities, we probed the impact of different resolutions by performing Meundon PDR modeling of [C II] $158 \mu \mathrm{m}$, [O I] $145 \mu \mathrm{m}$, and FIR emission for one pixel (chosen as the one where the $G_{\mathrm{UV}}$ distribution in Chevance et al. 2016 peaks), but on 12" scales. For this purpose, we used the same model grid with $A_{\mathrm{V}}=2 \mathrm{mag}$, as well as the same fitting method, as in Sect. 5.1.3 and constrained $P / k_{\mathrm{B}}=7 \times 10^{5} \mathrm{~K} \mathrm{~cm}^{-3}, G_{\mathrm{UV}}=3 \times 10^{4}$, and $\Omega=0.7$. These results are in good agreement with what Chevance et al. (2016) estimated $\left(P / k_{\mathrm{B}}=10^{6} \mathrm{~K} \mathrm{~cm}^{-3}, G_{\mathrm{UV}}=3 \times 10^{4}\right.$, and $\left.\Omega=0.5\right)$, essentially suggesting that the discrepancy between our study and Chevance et al. (2016) mostly results from the difference in the angular resolution. As Chevance et al. (2016) pointed out as well, utilizing lower resolution data skews the PDR solutions toward lower $P$ and $G_{\mathrm{Uv}}$, since the intensities measured on larger scales tend to be more dominated by the emission from diffuse regions with less UV illumination. 
M.-Y. Lee et al.: Radiative and mechanical feedback into the molecular gas in the Large Magellanic Cloud. II. 30 Doradus
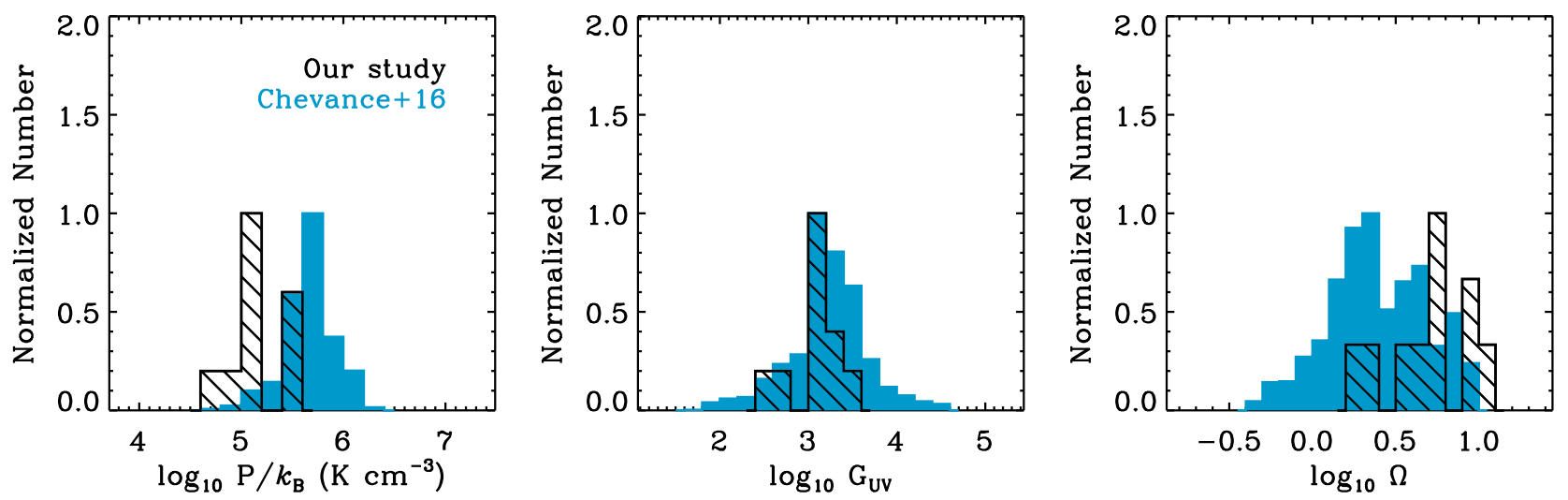

Fig. D.1. Comparison with Chevance et al. (2016). The normalized histograms of the PDR parameters in our study (42" scales) and Chevance et al. (2016) (12" scales) are shown in black (hatched) and blue (solid) respectively ( $P / k_{\mathrm{B}}, G_{\mathrm{UV}}$, and $\Omega$ on the left, middle, and right panels).

Appendix E: Comparison with Okada et al. (2019)

Recently, Okada et al. (2019) presented an independent study on $\mathrm{CO}\left(\mathrm{CO} J=2-1,3-2,4-3\right.$, and $6-5$, as well as ${ }^{13} \mathrm{CO} J=3-2$; observed with APEX) and fine-structure lines ([C II] $158 \mu \mathrm{m}$, [C I] $609 \mu \mathrm{m}$ and $370 \mu \mathrm{m}$, and [O I] $63 \mu \mathrm{m}$ and $145 \mu \mathrm{m}$; observed with APEX and SOFIA) in 30 Doradus. Among the observed transitions, $\mathrm{CO},{ }^{13} \mathrm{CO}$, [C II] and [C I] were mapped over an area that is comparable to our FTS coverage, while [O I] was obtained for selected positions only. This study based on high spatial $\left(\sim 6^{\prime \prime}\right.$ to $\left.\sim 30^{\prime \prime}\right)$ and spectral $\left(\sim 1 \mathrm{~km} \mathrm{~s}^{-1}\right)$ resolution data is complementary to our work, and we provide here a summary of it.

First of all, their high-resolution data clearly demonstrate the complexity of the neutral ISM in 30 Doradus. For example, the authors found that $\mathrm{CO},{ }^{13} \mathrm{CO}$, and [C I] spectra are similar, while [C II] $158 \mu \mathrm{m}$ shows a wider line width and/or additional velocity components. In addition, [O I] spectra match $\mathrm{CO}$ spectra at some locations, but they are more similar to [C II] $158 \mu \mathrm{m}$ profiles at other locations. In terms of spatial distribution, [C II] $158 \mu \mathrm{m}$ and CO $J=4-3$ show relatively similar structures, except for several mismatching peaks.

The complexity of the neutral ISM in 30 Doradus was also manifested in KOSMA- $\tau$ PDR modeling by Okada et al. (2019). The KOSMA- $\tau$ model calculates the thermal and chemical structures of a PDR as the Meudon PDR model does, but with a different geometry of the medium. Specifically, while the Meudon PDR model considers a plane-parallel slab of gas and dust, the KOSMA- $\tau$ model assumes an ensemble of clumps with a power-law mass spectrum $\mathrm{d} N / \mathrm{d} M \propto M^{-\alpha}$ ( $\alpha=1.8$ was used in Okada et al. 2019) to take into account the clumpiness of the ISM. Line and continuum intensities are then estimated by adding the contribution from each clump for a model grid of three parameters, total mass $\left(M_{\text {tot }}\right)$, average gas density $(\bar{n})$, and UV radiation field $\left(G_{\mathrm{UV}}^{\prime}\right)$. For more details on the KOSMA- $\tau$ PDR model, we refer to Stoerzer et al. (1996) and Röllig et al. (2006). While we cannot make a direct comparison with Okada et al. (2019) due to systematic differences in PDR modeling (e.g., input parameters and modeling approach), our results are essentially consistent in that both our study and Okada et al. (2019) show that one ISM component is not sufficient to analyze 30 Doradus. For example, Okada et al. (2019) modeled their observed transitions along with dust continuum emission for 30 Dor-10 at $(\alpha, \delta)_{\mathrm{J} 2000}=\left(05^{\mathrm{h}} 38^{\mathrm{m}} 48.8^{\mathrm{s}},-69^{\circ} 04^{\prime} 42.1^{\prime \prime}\right)$ and found that $\mathrm{CO}$ and $[\mathrm{C} \mathrm{I}]$ are relatively well reproduced, while [C II] and $\left[\begin{array}{ll}\mathrm{O} & \mathrm{I}\end{array}\right]$ are overestimated by a factor of a few. This bestfit KOSMA- $\tau$ model however predicts the CO SLED to be flat already around at $J=6-5$, suggesting that the gas is not warm enough ( $J_{\mathrm{p}}=9-8$ in our FTS observations). The constrained UV radiation field ( $\sim 200$ Draine fields) is indeed weaker than what we estimated for the low- and high- $P$ PDR components $\left(\sim 3 \times 10^{3}\right.$ Draine fields). In addition, the large beam filling factor of $\gtrsim 1$ is not consistent with what the ALMA $\mathrm{CO}(2-1)$ observations suggest (Indebetouw et al. 2013). All in all, both our study and Okada et al. (2019) highlight that many high-resolution tracers of gas and dust are required to build a consistent picture of the multi-phase, multi-component ISM in complex regions like 30 Doradus. 